Florida International University FIU Digital Commons

$8-29-2012$

\title{
The Endocrine Basis for Reproductive Life-history Trade-offs during the Previtellogenic Resting Stage in the Yellow Fever Mosquito, Aedes aegypti
}

Mark E. clifton

Florida International University, markec925@aol.com

DOI: $10.25148 /$ etd.FI12110706

Follow this and additional works at: https://digitalcommons.fiu.edu/etd

\section{Recommended Citation}

clifton, Mark E., "The Endocrine Basis for Reproductive Life-history Trade-offs during the Previtellogenic Resting Stage in the Yellow Fever Mosquito, Aedes aegypti" (2012). FIU Electronic Theses and Dissertations. 721.

https://digitalcommons.fiu.edu/etd/721 


\title{
FLORIDA INTERNATIONAL UNIVERSITY
}

\author{
Miami, Florida
}

\section{THE ENDOCRINE BASIS FOR REPRODUCTIVE LIFE-HISTORY TRADE-OFFS DURING THE PREVITELLOGENIC RESTING STAGE IN THE YELLOW FEVER MOSQUITO, AEDES AEGYPTI}

A dissertation submitted in partial fulfillment of the requirements for the degree of DOCTOR OF PHILOSOPHY

in

BIOLOGY

by

Mark Ellis Clifton 
To: Dean Kenneth G. Furton

College of Arts and Sciences

This dissertation, written by Mark Ellis Clifton, and entitled The Endocrine Basis for Reproductive Life-history Trade-offs during the Previtellogenic Resting Stage in the Yellow Fever Mosquito, Aedes aegypti, having been approved in respect to style and intellectual content, is referred to you for judgment.

We have read this dissertation and recommend that it be approved.

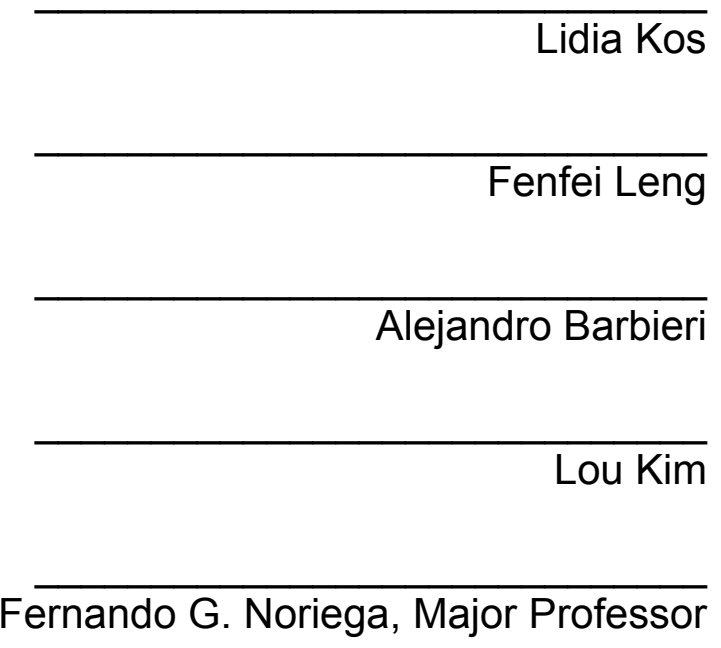

Date of defense: August, 29th 2012

The dissertation of Mark Ellis Clifton is approved.

Dean Kenneth G. Furton

College of Arts and Sciences

Dean Lakshmi N. Reddi University Graduate School

Florida International University, 2012 


\section{ACKNOWLEDGMENTS}

I wish to thank all the current and former members of the Noriega lab. Without their patient ears and critical insights none of this work would have been possible. I would especially like to thank Dr. Fernando Noriega for allowing me the latitude and providing me the support to pursue this project as well as Dr. Marcela Nouzova for her tireless rearing of mosquitoes. The members of my committee, Dr. Lidia Kos, Dr. Lou Kim, Dr. Fenfei Leng and Dr. Alejandro Barbieri, were instrumental in completing this work- thank you again! I would also like to thank Florida International University for awarding me the Presidential Enhanced Assistantship as well as the MBRS Biomedical Research Initiative summer award which made this work possible. Donors such as Ralph Sanchez also deserve acknowledgment for generously providing funding opportunities through the Ralph Sanchez Grand Prix scholarship. Finally, I would like to gratefully acknowledge Elsevier for permission to reprint chapters 3 and 4 which originally appeared in the Journal of Insect Physiology volume 57 issue 9, and volume 58 issue 7 , respectively. 


\begin{abstract}
OF THE DISSERTATION
THE ENDOCRINE BASIS FOR REPRODUCTIVE LIFE-HISTORY TRADE-OFFS DURING THE PREVITELLOGENIC RESTING STAGE IN THE YELLOW FEVER MOSQUITO, AEDES AEGYPTI
\end{abstract}

by

Mark Ellis Clifton

Florida International University, 2012

\author{
Miami, Florida \\ Professor Fernando Noriega, Major Professor
}

Juvenile hormone $(\mathrm{JH})$ is the central hormonal regulator of life-history trade-offs in many insects. In Aedes aegypti, JH regulates reproductive development after emergence. Little is known about JH's physiological functions after reproductive development is complete or $\mathrm{JH}$ 's role in mediating life-history trade-offs. By examining the effect of hormones, nutrition, and mating on ovarian physiology during the previtellogenic resting stage, critical roles were determined for these factors in mediating life-history trade-offs and reproductive output. The extent of follicular resorption during the previtellogenic resting stage is dependent on nutritional quality. Feeding females a low quality diet during the resting stage causes the rate of follicular resorption to increase and reproductive output to decrease. Conversely, feeding females a high quality diet causes resorption to remain low. The extent of resorption can be increased by separating the ovaries from a source of $\mathrm{JH}$ or decreased by exogenous application of methoprene. Active caspases were localized to resorbing follicles indicating that an apoptosis- 
like mechanism participates in follicular resorption. Accumulations of neutral lipids and the accumulation of mRNA's integral to endocytosis and oocyte development such as the vitellogenin receptor $(A a \vee g R)$, lipophorin receptor (AaLpRov), heavy-chain clathrin $(\mathrm{AaCHC})$, and ribosomal protein L32 (rpL32) were also examined under various nutritional and hormonal conditions. The abundance of mRNA's and neutral lipid content increased within the previtellogenic ovary as mosquitoes were offered increasing sucrose concentrations or were treated with methoprene. These same nutritional and hormonal manipulations altered the extent of resorption after a blood meal indicating that the fate of follicles and overall fecundity depends, in part, on nutritional and hormonal status during the previtellogenic resting stage. Mating female mosquitoes also altered follicle quality and resorption similarly to nutrition or hormonal application and demonstrates that male accessory gland substances such as $\mathrm{JH}$ III passed to the female during copulation have a strong effect on ovarian physiology during the previtellogenic resting stage and can influence reproductive output. Taken together these results demonstrate that the previtellogenic resting stage is not an inactive period but is instead a period marked by extensive life-history and fitness trade-offs in response to nutrition, hormones and mating stimuli. 


\section{TABLE OF CONTENTS}

CHAPTER

PAGE

1. Introduction 1

1.1 Dengue fever, insecticide resistance, and the need for new vector management techniques................................................................ 1

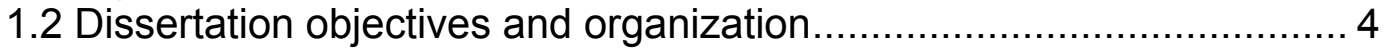

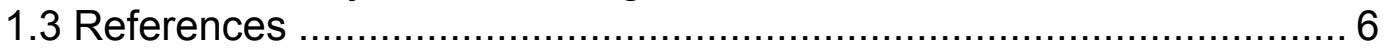

2. Juvenile hormone, life-history trade-offs and the reproductive endocrinology of Aedes aegypti.

2.1 Reproductive maturation, oogenesis and juvenile hormone synthesis

in the mosquito

2.2 Life-history trade-offs in insects are conflicts between germ and soma and are mediated by juvenile hormone .............................................. 13

2.3 Reproductive resources are reallocated through follicular

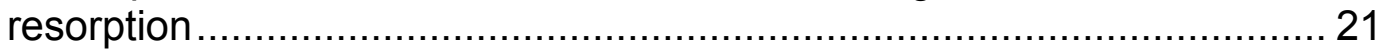

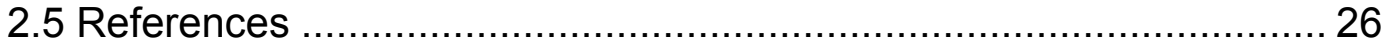

3. Nutrient limitation results in juvenile hormone mediated resorption of follicles

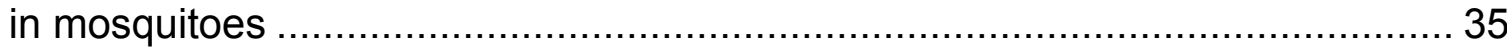

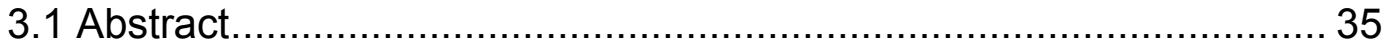

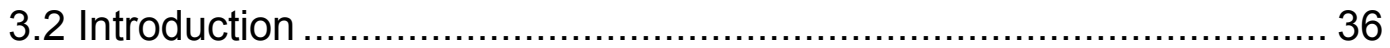

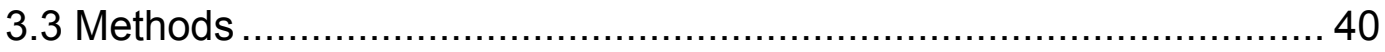

3.3.1 Insects........................................................................ 40

3.3.2 Observations of oosorption in previtellogenic ovaries .......... 40

3.3.3 Abdominal ligations .................................................... 41

3.3.4 Hormonal manipulations of intact mosquitoes.................... 42

3.3.5 Active caspase in situ assay .......................................... 42

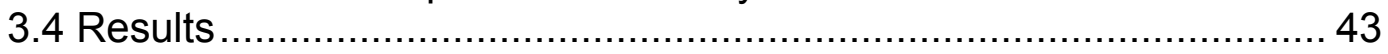

3.4.1 Nutrition affects previtellogenic follicular resorption ............. 43

3.4.2 Methoprene prevents follicular resorption in

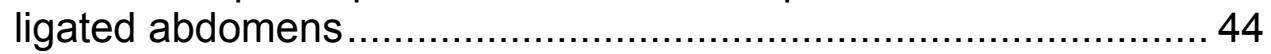

3.4.3 Methoprene prevents follicular resorption in

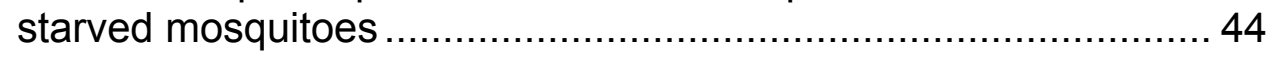

3.4.4 Active caspase in-situ assay ...................................... 45

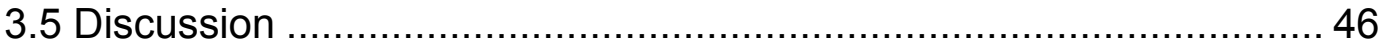

3.5.1 Nutrition, JH synthesis, and follicular resorption are linked .. 46

3.5.2 Follicular resorption as a resource allocation decision ......... 49

3.5.3 Follicular resorption as a life-history strategy ...................... 52

3.5.4 Progression of follicular resorption in previtellogenic

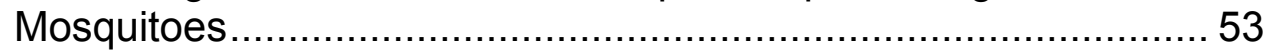

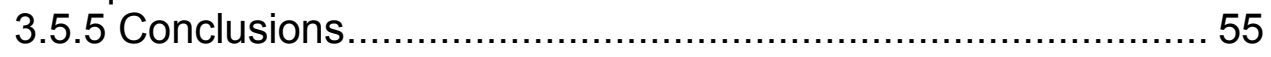

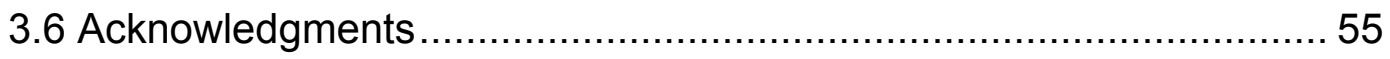


4. The fate of follicles after a blood meal is dependent on previtellogenic nutrition

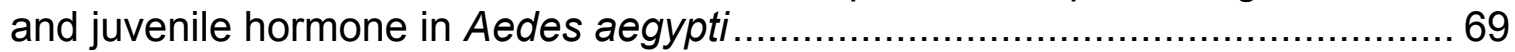

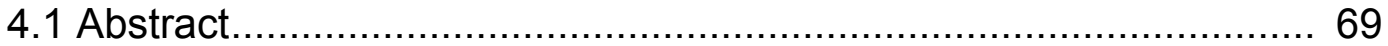

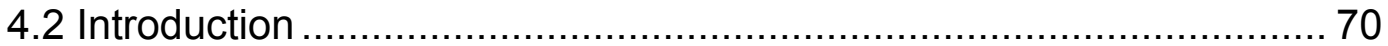

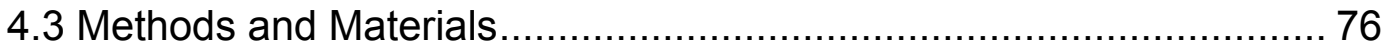

4.3.1 Nutritional manipulations of insects ................................... 76

4.3.2 Hormonal manipulations of insects .................................. 76

4.3.3 Ovarian triglyceride assay .............................................. 77

4.3.4 Ovarian micrographs and staining with Oil red-O ................. 77

4.3.5 Quantitative Realtime qPCR ........................................ 78

4.3.6 Determination of blood meal mass .................................... 79

4.3.7 Observations of ovarian resorption and final egg output ...... 80

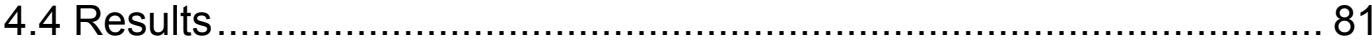

4.4.1 Ovary morphology reflects overall nutritional reserves......... 81

4.4.2 Previtellogenic nutrition alters neutral lipid content of

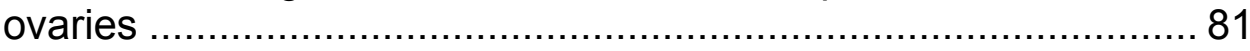

4.4.3 Previtellogenic nutrition alters expression of genes important for vitellogenesis .............................................................. 82

4.4.4 Previtellogenic methoprene application increases stored ovarian lipids

4.4.5 Previtellogenic methoprene application alters expression of genes important for vitellogenesis ......................................... 84

4.4.6 The fate of follicles after a blood meal is affected by previtellogenic nutrition ....................................................... 84

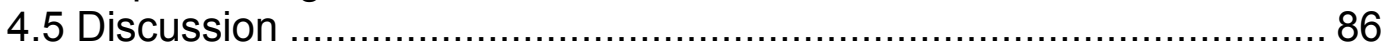

4.5.1 Defining oocyte and follicle quality .................................. 86

4.5.2 Oocyte lipids can be altered by previtellogenic nutrition as well as hormonal manipulation ............................................. 87 4.5.3 Previtellogenic expression of the endocytotic complex can be altered by nutrition and methoprene application .......................... 90 4.5.4 Low reserve mosquitoes take more blood but develop fewer eggs

4.5.5 A model for understanding the coordination of reproductive output with nutrition in the anautogenic mosquito ........................ 94

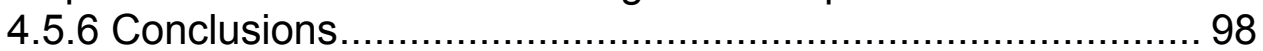

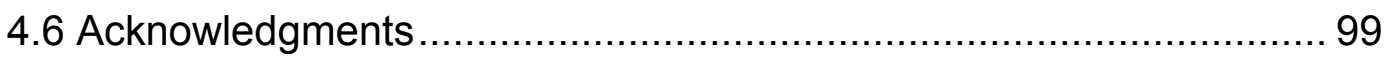

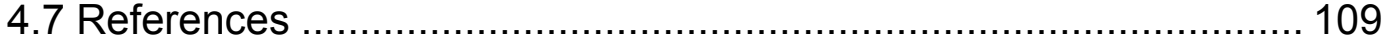

5. Male Aedes aegypti use JH III transferred during copulation to influence previtellogenic ovary physiology and affect the reproductive output of female

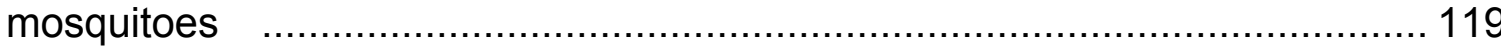

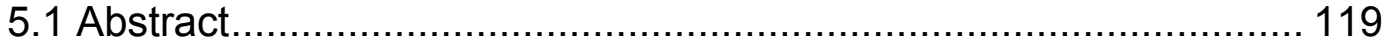

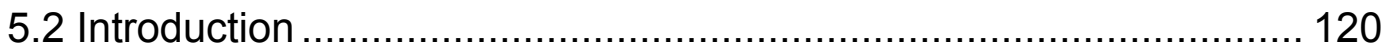




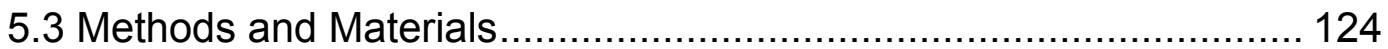

5.3.1 Insects and Mating …................................................. 124

5.3.2 Measurements of follicular resorption and ovarian lipids.... 125

5.3.3 Accessory gland injections ............................................ 125

5.3.4 Measurements of $\mathrm{JH}$ titers in male accessory glands, and female bursa copulatrix/spermathecae complexes ................... 126

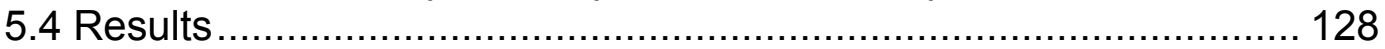

5.4.1 Mating reduces the rate of previtellogenic resorption......... 128

5.4.2 Mating increases previtellogenic lipid content of follicles ... 128

5.4.3 Male nutritional history affects previtellogenic ovary lipid content, the previtellogenic rate of resorption as well as reproductive output

5.4.4 Male accessory gland substances increase lipid content and

reduce resorption 130

5.4.5 Male mosquitoes transfer $\mathrm{JH}$ III to females during

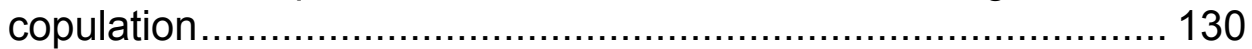

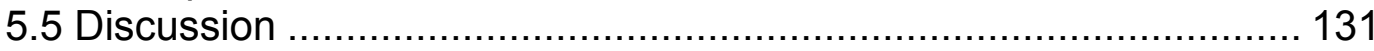

5.5.1 JH pleiotropy and resource allocation ............................ 131

5.5.2 Male accessory gland substances alter the resource allocation balance within the female insect .............................. 132 5.5.3 The male mosquitoes nutritional history alters the magnitude of the effect on female reproductive physiology ........................ 137

5.5.4 Conclusion ........................................................... 140

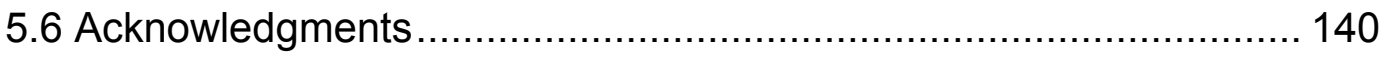

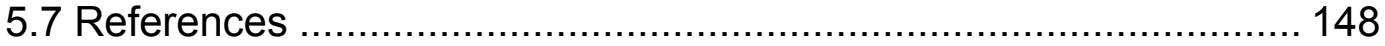

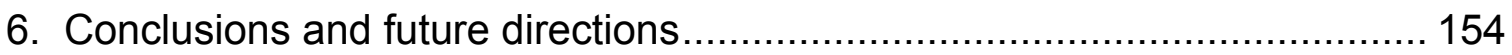

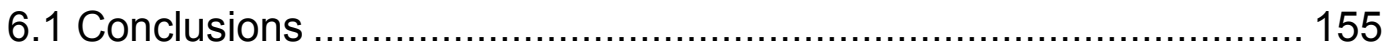

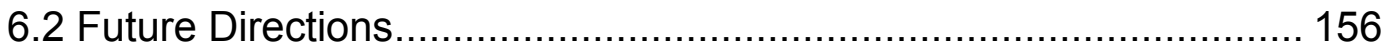

VITA 


\section{LIST OF FIGURES}

FIGURE PAGE

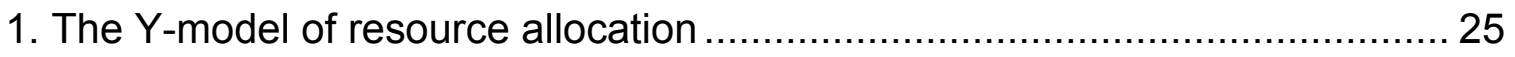

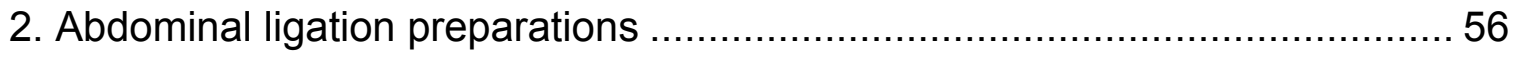

3. Normal and resorbing follicles stained with neutral red ............................. 57

4. Changes in the rate of previtellogenic follicular resorption .......................... 58

5. Effect of nutrition on the rate of previtellogenic follicular resorption................59

6. Methoprene prevents follicular resorption in ligated abdomens....................60

7. Methoprene prevents follicular resorption in starved mosquitoes .................. 61

8. Active caspase localization in previtellogenic follicles ................................ 62

9. Previtellogenic ovary morphology reflects previtellogenic nutritional

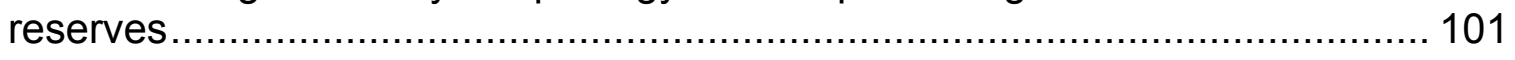

10. Lipid accumulation in the follicle increases in mosquitoes offered $20 \%$

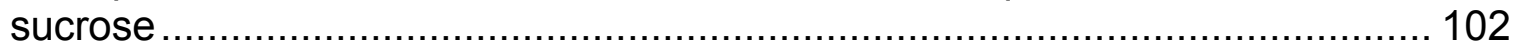

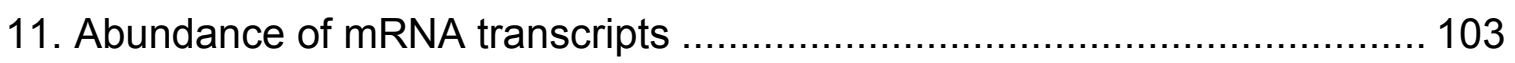

12. Lipid accumulation in the follicle increases when mosquitoes are treated

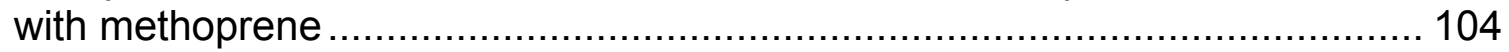

13. The abundance of mRNA transcripts in the ovaries increases when mosquitoes are treated with methoprene

14. Mosquitoes offered higher concentrations of sucrose take significantly smaller blood meals.

15. Sugar feeding or hormone treatment during the previtellogenic resting stage alters the rate of resorption 24 hours after a blood meal as well as total egg output.

16. A model that describes the effect of nutrition and hormones during the previtellogenic resting stage on follicular resorption after a blood meal. 
17. The average number of viable follicles decreases during previtellogenic development and vitellogenic development

18. Mating decreases the average rate of resorption in females maintained on $3 \%$ sucrose and $20 \%$ sucrose

19. The neutral lipid content of the previtellogenic ovary increases after mating in females maintained on $3 \%$ sucrose

20. Males maintained on $20 \%$ sucrose exert a larger effect on the rate of follicular resorption and neutral lipid content of ovaries

21. When females are mated to males maintained on $20 \%$ sucrose reproductive output is increased by $\sim 9$ eggs

22. Effect of injected accessory gland homogenates

23. $\mathrm{JH}$ III in the accessory gland of virgin males fed $3 \%$ sucrose and $20 \%$ sucrose as well as mated males fed $20 \%$ sucrose

24. The bursa copulatrix/ spermathecae complex contains more JH III in mated females than unmated females 


\section{LIST OF ABBREVIATIONS AND ACRONYMS}

mRNA Messenger ribonucleic acid

RNA Ribonucleic acid

DNA Deoxyribonucleic acid RER Rough endoplasmic reticulum $\mathrm{JH}$ Juvenile hormone CA Corpora allata DDT Dichlorodiphenyltrichloroethane WHO ...World Health Organization APS Aedes physiological saline SR-VAD-FMK sulforhodamine valylalanylaspartic acid fluoromethyl ketone PBS Phosphate buffered saline qPCR Real-time polymerase chain reaction OD Optical density ATP Adenosine triphosphate $A a V^{2}$ Aedes aegypti vitellogenin receptor AaLpRov Aedes aegypti ovarian lipophorin receptor $\mathrm{AaCHC}$ Aedes aegypti clathrin heavy chain rpL32 Ribosomal protein subunit L32 AeDronc Aedes aegypti homologue of Drosophila Nedd2-like caspase JHBP Juvenile hormone binding protein HPLC-FD. .High pressure liquid chromatography with fluorescent detection 
DBD-COCl 4-(n,n-Dimethylaminosulfonyl)

-7-(n-chloroformylmethyl-N-methylamino)-2,1,3-benzoxadiazole

BC-S …............................................ Bursa Copulatrix/ spermathecae complex

HEPES ........................................hydroxyethyl piperazineethanesulfonic acid 


\section{Chapter 1: Introduction}

\subsection{Dengue fever, insecticide resistance and the need for new vector management techniques}

It is estimated that nearly two-fifths of the world's population is now at risk for mosquito-borne dengue fever; a number which is likely to increase substantially as a consequence of climate, demographic and land-use changes (Hales et al., 2002; WHO, 2009). Some projections estimate that by 2055 , with a much warmer global average temperature and significant urbanization, more than a third of the world's future population, 3.2 billion people, will face an increased risk of dengue fever infection including large portions of the United States (Hales et al., 2002). As if to accentuate the public health threat to the United States, a health advisory was issued during the summer of 2010 by the Centers for Disease Control and local county health departments for Puerto Rico and Key West after sustained transmission of the disease was confirmed (CDC, $2010 a, b)$. These cases represent the first sustained transmission of this disease in Florida since 1934 (CDC, 2010a).

Measuring the economic impact of a disease like dengue fever is difficult because of the inadequate surveillance measures employed by the governments of afflicted nations. According to the World Health Organization, dengue fever's economic costs to society are comparable to tuberculosis, malaria, hepatitis or bacterial meningitis (Gubler, 2002). Surprisingly, this estimate does not consider the economic costs of controlling the vector Aedes aegypti, the environmental costs of insecticide application nor the personal and economic costs to 
individuals. As a result of the specific characteristics of dengue's mode of transmission (through a bite from an infected mosquito), dengue fever is often socioeconomically distributed with a greater incidence in those most likely to be outdoors, in dense urban areas with standing water and poor sanitation as well as those without access to adequate protection measures (Mondini and Chiaravalloti-Neto, 2007; Almeida et al., 2009).

Compounding the threat posed by dengue is the growing resistance of $A$. aegypti and other insects to many common classes of insecticides including DDT, pyrethroids, endocrine disruptors, carbamates and organophosphates (Hemmingway and Ranson, 2000; Pereira Lima et al., 2003; Hemmingway, 2007). Nearly one-hundred species of mosquitoes as well as species of fleas, ticks, lice, bedbugs and others have become resistant to insecticides (WHO, 1992). Many of the most commonly used insecticides have become diminished in their capacity to control populations of target species (Hemmingway and Ranson, 2000). To remain effective, many vector control programs must now monitor insect resistance while rotating between classes of insecticides to achieve results (WHO, 2009). Despite efforts to manage vector resistance, A. aegypti shows cross-resistance between classes of insecticides to the point where the use of one class of insecticide may exclude the later use of another (WHO, 2009). Coupled with dwindling industrial investment in new insecticides, the list of useable compounds for vector control is quickly narrowing (Zaim and Guillet, 2002). During the last twenty years, not one new class of insecticides has been 
made available for indoor spraying or mosquito net treatment (Zaim and Guillet, 2002).

Even relatively "safe" insecticides may have deleterious effects on nontarget organisms (i.e., bees) or may enter the food supply when applied near agricultural zones (Rose, 2001). Commonly used organophosphates, in particular, have been demonstrated to be linked to a variety of neurodevelopmental defects in animals and possibly other neurological conditions in children (For a review see Eskenazi et al., 1999). Other effects of long-term or complex synergistic exposures to insecticides or groups of insecticides are currently unknown but potentially dangerous. It is hoped that by specifically targeting future classes of insecticides with detailed endocrinology information, toxicology and off-target effects can be largely circumvented (Zaim and Guillet, 2002). New insecticides that are safe and effective are considered crucial in the battle against malaria and dengue and will also reduce our dependence on chemicals known to be toxic and damaging to the environment (Zaim and Guillet, 2002). However, these insecticides cannot be developed without a full understanding of the endocrinology of this disease vector.

The worldwide spread of dengue fever has demonstrated that conventional vector control strategies have not been adequate to stop the spread of the yellow fever mosquito, Aedes aegypti. The triad of disease, insect resistance, and insecticide toxicity has underscored the need for a new generation of vector management strategies based on detailed biochemical, endocrinological and physiological studies. Therefore, basic information about 
the reproductive endocrinology of mosquitoes and other vectors of disease has never been more important.

\subsection{Dissertation objectives and organization}

Juvenile hormone has been characterized as the central endocrine mediator of life-history and developmental trade-offs among insects. Because of this important role, many vector management strategies have relied on the disruption or manipulation of $\mathrm{JH}$ signaling to impair the reproductive or developmental physiology of insects. Although much is known about the function of $\mathrm{JH}$ in insects, there are still many aspects of the hormonal regulation of mosquito reproduction that have not been described. The role of $\mathrm{JH}$ during the previtellogenic resting period in particular has been consistently overshadowed as an object of study by other, more dramatic endocrinological events such as the maturation period directly following emergence or the period of intense oogenesis after a blood meal. Because of this, the previtellogenic resting stage in $A$. aegypti has been studied very little and has generally been thought of as a developmental quiescence. However, exploring this stage through the lens of life-history theory has revealed that $A$. aegypti possesses a uniquely complicated reproductive physiology and life-history strategy that is more closely connected to events before a blood meal than previously understood.

Since A. aegypti has emerged as an important laboratory model and also a widespread vector of disease, understanding the full reproductive cycle of this mosquito is vital to future management strategies and provides a valuable context for future scientific discoveries. Therefore, the aim of this dissertation is 
to determine the relationships between juvenile hormone, nutritional status, and the male contribution to reproduction and fecundity during a physiological period previously thought to be a developmental quiescence (i.e., "resting stage"). The completion of these aims has demonstrated that the previtellogenic resting stage in A. aegypti is not a "resting" stage at all, but is instead a period marked by a constant and dynamic coordination of reproductive output determined by nutritional, hormonal and other biotic factors.

My dissertation begins to describe the endocrine basis for the life-history, reproductive and resource allocation trade-offs that determine mosquito fecundity and ultimately contribute to the incidence of disease. Chapter 2 reviews the literature upon which this dissertation rests and describes both the reproductive endocrinology of Aedes aegypti as well as the broader work that explores the endocrinology of life-history trade-offs and resource allocations in insects. Chapter 2 ends with a section that examines the unique role follicular resorption likely plays in current resource allocation schemes. Chapters 3 and 4 are both reprints of articles previously published in the Journal of Insect Physiology. Chapter 3 explores the endocrinology of follicular resorption in previtellogenic mosquitoes and demonstrated that sugar feeding during the previtellogenic resting stage can prevent resorption and enhance fecundity. Chapter 4 examines how $\mathrm{JH}$ action and sugar feeding during the previtellogenic resting stage determines reproductive output after a blood meal. A model is also presented in chapter 4 that illustrates how previtellogenic nutrition and blood meal derived nutrition are integrated through $\mathrm{JH}$ signaling to determine 
reproductive output. Chapter 5 further examines reproductive output of the female mosquito in terms of the male contribution and the model presented in chapter 4. Finally, chapter 6 summarizes the important observations of the research presented in chapters 3-5 and describes the next investigative steps in this line of research.

\subsection{References}

Almeida, A.S., Andrade Mendronho, R., Ortiz-Valencia, L.I. 2009. Spatial analysis of dengue and the socioeconomic context of the city of Rio de Janeiro Southeastern Brazil). Rev. Saúde Pública. 43, 4.

Centers for Disease Control. 2010a. Locally Acquired Dengue-Key West, Florida, 2009-2010. MMWR 2010. 59, 577-581.

Centers for Disease Control. 2010b. Increased Potential for Dengue Infection in Travelers Returning from International and Selected Domestic Areas. CDC Health Advisory.

Eskenazi, B., Bradman, A., Castorina, R. 1999. Exposures of children to organophosphate pesticides and their potential adverse health effects. Environmental Health Perspectives. 07, 409-419.

Gubler, D.J. 2002. Epidemic dengue/dengue hemorrhagic fever as a public health, social, and economic problem in the 21st century. Trends in Microbiology. 10, 100-103.

Hales, S., de Wet, N., Maindonald, J., Woodward, A. 2002. Potential effect of population and climate changes on global distribution of dengue fever: an empirical model. Lancet. 360, 830-834.

Hemmingway, J., Ranson, H. 2000. Insecticide resistance in insect vectors of human disease. Annual Reviews. 45, 371-391.

Hemmingway, J. 2000. The molecular basis of two contrasting metabolic mechanisms of insecticide resistance. Insect Biochemistry and Molecular Biology. 30, 1009-1015.

Hemingway, J., Beaty, B.J., Rowland, M., Scott, T.W., Sharp, B.L. 2006. The Innovative Vector Control Consortium: improved control of mosquito-borne diseases. Trends in Parasitology. 22, 308-312. 
Mondini, A., Chiaravalloti, B.L. 2007. Socioeconomic variables and dengue transmission. Rev. Saúde Pública. 41(6).

Pereira Lima, J.B., Pereira Da-Cunha, M.P., Carneiro Da Silva Junior, R., Ribeiro Galardo, A.K., Da Silva Soares, S., Aparacida Braga, I., Pimentel Ramos, R., Valle, D. 2003. Resistance of Aedes aegypti to organophosphates in several municipalities in the state of Rio De Janeiro and Espirito Santo, Brazil. American Society for Tropical Medicine and Hygiene. 68, 329-333.

Rose, R.I. 2001. Pesticides and public health: Integrated methods of mosquito management. Emerging and Infectious Diseases. 7, 17-23.

World Health Organization. 2009. Dengue and dengue hemorrhagic fever. WHO fact sheet. No. 117.

World Health Organization 1992. Vector resistance to pesticides. 15th Report of the WHO Expert Committee on Vector Biology and Control. WHO/TRS/818.

Zaim, M., Guillet, P. 2002. Alternative insecticides: an urgent need. Trends in Parasitology. 18, 161-163. 


\section{Chapter 2: Juvenile hormone, life-history trade-offs and the reproductive}

endocrinology of Aedes aegypti

\subsection{Reproductive maturation, oogenesis and juvenile hormone synthesis in the mosquito}

Juvenile hormone $(\mathrm{JH})$ was first described by Sir Vincent Wigglesworth in 1934. Using Rhodnius spp. and surgical parabiosis techniques, Wigglesworth described the presence of a factor secreted by the corpora allata of insects that regulates development and metamorphosis (Gilbert et al., 2000). Wigglesworth called his factor juvenile hormone, and over 30 years later the chemical structure was elucidated by Röller (Röller et al., 1967; Gilbert et al., 2000). Later work by Nijhout and Williams (1974) established JH as the central gonadotropic hormone in the tobacco hornworm moth, Manduca sexta. Using purified JH as well as an array of bioactive $\mathrm{JH}$ analogs, researchers have reaffirmed $\mathrm{JH}$ 's primary roles as a developmental regulator and gonadotropin across a wide variety of insect taxa (Riddiford, 1996). The roles of $\mathrm{JH}$ as a developmental regulator and gonadotropin have been widely reviewed (Engelmann, 1968; Riddiford, 1980; Riddiford, 1996; Strambi et al., 1997; Truman and Riddiford, 2002).

Since the initial description of a juvenile hormone in $M$. sexta, additional $\mathrm{JH}$-like compounds have been described that differ structurally in the placement of methyl groups and/or epoxide rings. As a group, all juvenile hormones can be characterized as acyclic sesquiterpenoid compounds synthesized de novo by the corpora allatum (CA). However, $\mathrm{JH}$ III (referred to hereafter as only $\mathrm{JH}$ ) is the only juvenile hormone produced by the corpora allata in female Aedes aegypti. 
Although limited evidence exists that additional JH's (namely, JH I and $\mathrm{JH}$ III bisepoxide) may be produced by male mosquitoes, it is not clear how or if these additional $\mathrm{JH}$ compounds are involved in reproduction in Aedes aegypti (Borovsky et al., 1994).

Juvenile hormone synthesis proceeds through 13 synthetic steps culminating in the secretion of a final hormone product (Noriega et al., 2006). The first 8 steps in the synthesis of all JH's (early steps) follow the mevalonate pathway common to many higher eukaryotes (Bellés et al., 2005). The remaining 5 steps (late steps) in $\mathrm{JH}$ synthesis are specific to insects and differ slightly among taxa (Noriega et al., 2006). Strongly hydrophobic, JH is generally found bound to a specific binding protein (JHBP) that enables transport in the aqueous environment of the haemolymph (Trowell, 1992). While the exact receptor-protein for $\mathrm{JH}$ is currently the subject of debate, $\mathrm{JH}$ clearly functions at the transcriptional level in a manner similar to the steroid hormones to alter the expression of target genes (Jones, 1995).

In $A$. aegypti, $\mathrm{JH}$ functions primarily as the central regulator of reproductive maturation (Feinsod and Spielman, 1980a; Feinsod and Spielman, 1980b; Hagedorn et al., 1977; Lea, 1963; Gwadz and Spielman, 1973; Klowden, 1997). Within 24 hours after eclosion, JH synthesis by the corpora allatum peaks at over $30 \mathrm{fmol} /$ pair $\mathrm{CA} /$ hour and initiates a variety of developmental events ( $\mathrm{Li}$ et al., 2003). In response to elevated JH synthetic rates after emergence, the oocyte differentiates from the nurse cells, the follicular epithelial cells multiply and the total size of the ovarian follicles more than doubles (Feinsod and Spielman, 
1980a, Hagedorn et al., 1977; Briegel, 1990). In addition to an increase in follicle size, mitochondria and rough endoplasmic reticulum (RER) proliferate within follicular epithelial cells, and an endocytotic complex consisting of receptor proteins and clathrin coated pits capable of supporting yolk protein incorporation develops (Clements and Boocock 1984; Raikhel and Lea, 1985; Raikhel and Lea, 1991). The accumulation of mRNA's within the nurse cells and oocytes for proteins important to oogenesis occurs during this time (Kokoza and Raikhel, 1997; Cho and Raikhel 2001; Cheon et al., 2001; Seo et al., 2003). Under the direction of $\mathrm{JH}$, a variety of cytological and developmental changes occur outside the ovaries including ribosomal and RER changes in fat body trophocytes, and increases in the abundance of midgut mRNA's related to blood digestion (Klowden, 1997; Raikhel and Lea, 1990; Dhadialla and Raikhel, 1994; Noriega et al., 1997).

Each of these developmental features contributes to an adult phenotype capable of blood feeding and reproduction. Most importantly, these morphological and cytological changes do not occur in mosquitoes decapitated or allatectomized immediately following emergence which demonstrates that the corpora allata produces $\mathrm{JH}$, and this $\mathrm{JH}$ is responsible for developing reproductive and blood feeding physiology in newly emerged mosquitoes (Feinsod and Spielman, 1980b; Raikhel and Lea, 1985; Raikhel and Lea, 1991; Lea 1963).

By 72 hours post-emergence, initial reproductive maturation is complete, $\mathrm{JH}$ synthesis decreases to $\sim 10 \mathrm{fmol} / \mathrm{CA}$ pair/hour (Li et al., 2003) and the 
mosquito enters a host-seeking previtellogenic resting stage (Clements, 1992). During the previtellogenic resting stage the corpora allata maintains a lower rate of $\mathrm{JH}$ synthesis until a blood meal is found. The resting stage can potentially last many days although in the laboratory it is often artificially limited to only a day or two by offering a blood meal. The fuel for flight and host seeking behavior during this time is primarily provided by nectar feeding in the wild or sugar feeding in the laboratory (reviewed in Foster, 1995). Sugar feeding also induces the synthesis and storage of triglycerides within the fat body (Nayar and Van Handel, 1971; Briegel, 1990; Briegel et al., 2001). Therefore, female mosquitoes can significantly add to lipid reserves during the resting stage provided adequate sugar meals are present. However, further oogenesis is always restrained until a sufficient blood meal is found (Clements, 1992).

Oogenesis recommences after a blood meal is ingested marking the end of the previtellogenic resting stage and the beginning of the vitellogenic stage (Clements, 1992). Synthesis of JH rapidly decreases to the lowest levels found during a gonotrophic cycle (Li et al., 2003). Neuropeptides released in response to abdominal distension from the blood meal induce the ovaries to synthesize large amounts of ecdysteroids (Gwadz, 1969; Greenplate et al., 1985). In response to elevated amino acids from the partially digested blood meal, targetof-rapamycin kinase (TOR) is phosphorylated (Hansen et al., 2004) and in concert with high ecdysteroid titers (Martin et al., 2001) activates the downstream transcription of genes such as vitellogenin, vitellogenic carboxypeptidase and lipophorin (reviewed in Raikhel and Dhadialla, 1992). These yolk proteins are 
secreted into the haemolymph, ingested by the oocyte via receptor-mediated endocytosis and incorporated into vitellin (Raikhel and Lea, 1985; Raikhel and Lea, 1991). Stored lipids carried into adulthood from the larval stage as well as those synthesized from sugar meals are mobilized from the fat body and are also incorporated into the oocyte via receptor mediated endocytosis (Zeigler and Ibrahim, 2000; Briegel et al., 2002; Zhou et al., 2004a). Since the eggs of $A$. aegypti require immersion in rainwater to hatch (Clements, 1992), stored yolkproteins and lipids provide the energy and amino acids to maintain a $1^{\text {st }}$ instar larva during a potentially extended quiescence (Van Handel, 1993).

In concert with intense yolk-protein synthesis and lipid transfer by the fat body, an increase in the synthesis of endocytotic components by the ovarian follicles also occurs after a blood meal. In this way, the manufacture of yolk proteins occurs in parallel with the capacity to import yolk components into the oocyte (Kokoza and Raikhel, 1997; Cho and Raikhel 2001; Cheon et al., 2001; Seo et al., 2003). By 72 hours after a blood meal, the nurse cells undergo apoptosis and add their contents to the oocyte. For a review of nurse cell dumping see McCall, (2004). The follicular epithelium secretes chorion proteins to surround the oocyte, and the follicular epithelial cells begin apoptosis. After all follicular epithelial cells have been removed, a batch of eggs is ready for deposition (Clements, 1992). Juvenile hormone synthesis begins again and initiates the maturation of a new batch of follicles to a second previtellogenic resting stage (Li et al., 2003). 


\subsection{Life-history trade-offs in insects are conflicts between soma and germ and are mediated by juvenile hormone}

Juvenile hormone was previously described as the "status quo" hormone because of its inhibitory effect on larval development in many species (Williams, 1961; Sláma and Williams, 1965; Nijhout and Williams 1974; Nijhout and Riddiford, 1974; Nijhout, 1975; Riddiford, 1996; Truman et al., 2006). According to Nijhout nearly 30 years ago, "The status quo action of $\mathrm{JH}$ has, until recently, been the only well-established role for this hormone during larval life." (1975). More recent work by Riddiford describes the primary role of $\mathrm{JH}$ as "...to prevent metamorphosis and to regulate reproductive maturation" (1996). While these general interpretations of JH's function have been upheld across many insect taxa, other roles for $\mathrm{JH}$ have been described that redefine the framework laid down by earlier researchers. Viewing JH solely as a developmental "status quo" hormone or a gonadotropic factor is probably no longer adequate. Newer research has indicated that a wide variety of physiological, developmental, and phenotypic trade-offs are regulated by JH (Flatt et al., 2005). As Flatt et al. points out, "...JH now emerges as an endocrine regulator of development, life history, and fitness trade-offs" (2005). Interestingly, the endocrinological basis for reproduction in $A$. aegypti has rarely been studied under such a conceptual framework.

All organisms face simultaneous and competing physiological and developmental demands. The trade-offs made in response to these competing demands become especially apparent when nutrition is limited or other 
environmental factors are less than ideal. Life-history and fitness tradeoffs in insects such as flight-oogenesis syndrome (reviewed in Dingle and Winchell, 1997), developmental polyphenisms (i.e., horned beetle polyphenism ) (Emlen, 1997; Emlen and Nijhout, 1999) lifespan/fecundity trade-offs (Tatar and Carey, 1995; Flatt and Kowecki, 2007) as well as follicular resorption represent adaptive life-history manifestations of the critical need to balance resource allocation between somatic and reproductive physiology. Patterns of resource allocation between reproductive and somatic physiology have been described in the literature in a variety of ways including germ/soma conflict theory and source-sink models (Heininger, 2002). Regardless of the terminology used to describe the phenomena, the pattern of resource allocation between reproductive and somatic physiology forms the basis for many observed life-history and fitness tradeoffs in insects and also underpin theories of senescence (reviewed in Boggs, 2009).

The relationship between incoming nutrition, reproduction and survival has also been described using a "Y" model of resource allocation (Figure 1; Jong and van Noordwijk, 1995; Tatar and Carey, 1995; reviewed in Harshman and Zera, 2007). Under this model, acquired resources entering at the stem of the "Y" join a pool of resources which can be allocated towards somatic/survival physiology on one branch of the "Y" or towards germ/reproductive physiology on the other (Figure 1). Manipulations to various components of this model are expected to lead to trade-offs elsewhere. For example, excessive allocations towards reproductive output usually lead to a decreased lifespan (Flatt et al. 2005; Tu et al. 2006) and as expected, experimental reductions in reproductive output have 
been shown to extend lifespan (Clancy et al. 2001; Tatar et al. 2001; Tu et al. 2005). Although alternative models that incorporate and expand the "Y" model have been suggested in an attempt to more fully describe the lifelong resource allocation decisions an insect is likely to face (reviewed in Harshman and Zera, 2007; reviewed in Boggs, 2009), it is generally believed that the relationship between nutrition, reproductive output and somatic physiology as described by the "Y" model remains fundamentally valid (Harshman and Zera, 2007; Boggs, 2009). By viewing well-known examples of $\mathrm{JH}$-mediated life-history trade-offs under the context of the "Y" model, it is clear that $\mathrm{JH}$, in many cases, plays a critical role in mediating the balance between somatic and reproductive physiology in insects and begins to provide clues to the mechanisms that govern resource allocations (Figure 1; reviewed in Dingle and Winchell, 1997; reviewed in Zera and Harshman, 2007; reviewed in Boggs, 2009).

In some cases the trade-off between soma and reproductive physiology is obvious and its endocrinological basis is clear. For example, in the wingpolymorphic bug, Pyrrhocoris apterus, application of the $\mathrm{JH}$ analog methoprene was found to alter the allocation of resources between soma and germ line cells during development (Socha and Sula, 2007). More specifically, methoprene caused winged morphs of this species to histolyse flight muscle in favor of reproductive tissue (Socha and Sula, 2007). P. apterus was specifically chosen because it exists in a winged and wingless morph and can be used to test the trade-off between two energetically expensive physiological processes; reproduction and flight (i.e., flight-oogenesis syndrome) (Socha and Sula, 2007). 
Implied by these results is an important role for $\mathrm{JH}$ in determining the life-history strategy in this species by altering the relationship between adult dispersal and reproduction through alterations in the allocation of resources between soma and germ during development (Zera and Cisper, 2001; Socha and Sula, 2007).

Work done with, Gryllus firmus, has also demonstrated that $\mathrm{JH}$ can act as a mediator of resource allocation between soma and germ with overarching implications for the life-history strategy of an insect. Like $P$. apterus, the cricket G. firmus also exists in winged and wingless forms which differ in whole-body $\mathrm{JH}$ titer. Each morph also differs in its dispersal and reproductive ability (Zera and Cisper, 2001). Wingless forms have a high JH titer, a higher reproductive ability but cannot disperse as far. Winged forms have a low JH titer, can disperse further but have a lower total reproductive output. In this example as well, the trade-off between two costly physiological activities (dispersal/flight physiology and reproduction) is mediated by $\mathrm{JH}$ through alterations in the allocation of resources to opposing physiological processes during development.

In the horned beetles, Onthophagus taurus and $\mathrm{O}$. acuminatus, $\mathrm{JH}$ mediates the phenotypic decisions that are integral to these species life-history strategy. Horned beetles are diphenic with respect to horn growth and mating behavior; males with horns engage in fighting for a mate, those without engage in sneaking (Moczek and Emlen, 2000). Males with horns always win fights against males without horns and males without horns tend to favor sneaking past horned males to gain access to a female (Moczek and Emlen, 2004). Upon application of $\mathrm{JH}$, larval males nutritionally competent to grow horns were rendered unable to 
grow horns (Nijhout and Emlen, 1998). However, the compound eyes of treated males grew significantly larger similarly to the hornless phenotype (Nijhout and Emlen, 1998). In a later study, destruction of the precursor cells to male genitalia in larval O. taurus caused the over-expression of head horns (Moczek and Nijhout, 2004). These results suggest two levels where JH plays an active role. On the first level, $\mathrm{JH}$ is mediating the relationship between developing body parts in a clear example of a developmental soma/germ trade-off by mediating the allocation of resources between gonadal development and head horns. On another level, JH appears to mediating the larger polyphenic decision of whether to grow horns at all which would then determine the long-term reproductive strategy (fighting vs. sneaking). Presumably each reproductive strategy and phenotype carries its own costs and benefits which are determined through the action of $\mathrm{JH}$ during larval development.

The extent to which $\mathrm{JH}$ produces various developmental outcomes does not exist arbitrarily. In many cases, the relationship between $\mathrm{JH}$ and nutritional status underlies the trade-off decisions that insects make. In the horned beetles, Onthophagus spp. the nutritional state of the larva also determines whether or not horns will grow (Emlen, 1997; Emlen and Nijhout, 1999). Larger and better fed male larvae will grow horns if they reach a certain critical threshold for body size. Males below the threshold will not grow horns (Emlen, 1997; Emlen and Nijhout, 1999). The "decision" to grow horns is made during development using body size and nutritional status as the deciding factor and $\mathrm{JH}$ as the inducer of the decision (Emlen and Nijhout, 1999). Juvenile hormone acts as the critical 
linkage between nutritional status, final adult phenotype, and therefore the reproductive strategy employed (Nijhout and Williams, 1974; Emlen, 1997; Emlen and Nijhout, 2001).

A role for $\mathrm{JH}$ that links nutritional status with physiological, developmental or reproductive outcomes is not without precedent in other taxa as well. Lubber grasshoppers, Romalea microptera, require $\mathrm{JH}$ to synthesize yolk proteins (Fronstin and Hatle, 2007). The synthesis of $\mathrm{JH}$ in this species is dependent on nutritional status (Fronstin and Hatle, 2007). Under nutritional limitation, JH production is halted and yolk protein synthesis and incorporation into the oocyte cannot occur (Fronstin and Hatle, 2007). However, there is an additional layer of complexity to the interpretation of $\mathrm{JH}$ 's role in this species. Nutritionally deprived females exposed to $\mathrm{JH}$ were found to commit to oviposition much sooner than nutritionally competent females. In other words, females with low nutritional reserves favored current reproduction and those females with adequate reserves favored future reproduction and this decision was mediated by $\mathrm{JH}$ (Fronstin and Hatle, 2007). On one level, JH mediates the physiological relationship between nutrition and egg development through its effects on yolk protein synthesis. By mediating the pattern of yolk synthesis and egg development, $\mathrm{JH}$ is also mediating long-term reproductive decisions and fitness trade-offs. The adjustment of reproductive timing in response to nutritional status and $\mathrm{JH}$ synthesis illustrates the dual nature of $\mathrm{JH}$ to regulate specific physiological events while simultaneously mediating longer-term life-history strategies (Fronstin and Hatle, 2007). 
While mosquitoes such as $A$. aegypti most certainly face developmental and life-history trade-offs similarly to other insects, these have been mostly obscured because of the particulars of a hemophagic anautogenic life-history strategy and also the failure to view mosquitoes within such a context. However, there is evidence that $\mathrm{JH}$ mediates some aspects of mosquito reproductive tradeoffs in a way that is comparable to other insects. For example, in $A$. aegypti the extent of previtellogenic ovarian development is dictated by nutritional status through JH synthesis. Smaller, less-nourished females do not initiate previtellogenic maturation directly after eclosion to the extent that larger well-fed females will (Gwadz and Spielman, 1973; Hagedorn et al., 1977; Feinsod and Spielman, 1980; Noriega, 2004; Caroci et al., 2004; Hernandez-Martinez et al., 2006; Shin-Hong et al., 2007). Like other insects, JH is believed to signal this development on the basis of nutritional signals from throughout the body (Caroci et al., 2004; Telang et al., 2004). Topical application of exogenous JH will induce small mosquitoes to undergo inappropriate maximum previtellogenic development despite a nutritional deficiency (Feinsod and Spielman, 1980a; Feinsod and Spielman, 1980b; Caroci et al., 2004). The correct timing of ovarian development is likely important in this species because committing resources to ovarian development with inadequate nutritional reserves may have detrimental effects (Caroci et al., 2004; Noriega, 2004; Telang et al., 2004). Since female mosquitoes can emerge in two distinct phenotypes (large and small) determined by larval nutritional state and $\mathrm{JH}$ regulates initial maturation and the extent of resources committed to reproduction, it would seem that in $A$. aegypti, JH also 
regulates the reproductive strategy of this species by managing ovarian development until the proper nutritional goals are reached. In this species as well, the relationship between the soma and germ, at least during initial reproductive development, is mediated by juvenile hormone. However, little else is known about JH's role in reproductive, developmental or life-history trade-offs in mosquitoes.

Juvenile hormone is not the only factor that has the potential to alter the balance between somatic and reproductive physiology. Male accessory gland (AG) substances, passed to the female during copulation, are usually a complex mixture of peptides, proteins, hormones and other substances that have profound effects on female physiology, behavior and reproduction. In $D$. melanogaster, male AG substances (specifically sex-peptide (Acp-70) and ovulin (Acp-26a)) have been shown to exert a variety of effects on the female reproductive physiology which are congruent with alterations in the pattern of resource allocations as understood by the "Y" model. Drosophilid male AG substances, have been shown to increase the rate of oogenesis (Chapman et al., 2003) stimulate JH synthesis (Moshitsky et al., 1996) and also reduce longevity in female flies (Wigby and Chapman, 2005). In other insects as well, the effects of male AG substances (and also nuptial gifts) on female physiology generally manifest in ways that appear as alterations or manipulations to the "Y" model or investments in a female's endogenous resource allocation balance (Boggs, 1990, Karlsson, 1998). Therefore, it seems that the key to understanding the nature of the male reproductive investment in mosquitoes (and possibly also the nature of 
sexual conflict in insects), is by viewing the male reproductive effort as an attempt to alter the resource allocation balance within a female.

\subsection{Reproductive resources are reallocated through follicular resorption}

When trade-offs between two physiological processes include reproductive activities as one of the trade-off partners, the resorption of ovarian follicles is commonly the result. Follicular resorption (also called oosorption or follicular atresia) is a process by which reproductive output can be reduced by active degeneration of ovarian follicles. In insects, resorption is a widespread phenomenon which can occur for a variety of reasons including, seasonality, nutrition, ovipositional block, or other biotic and abiotic factors (Reviewed in Bell and Bohm, 1975). Follicular resorption represents the only described mechanism in which the flow of resources allocated towards reproduction seems to be stopped or even reversed towards somatic processes and may represent a unique complication to the traditional "Y" model of resource allocation. To remain congruent with existing models of resource allocation, there appears to be three main hypotheses that describe the function of resources recovered from resorbing follicles: 1) to support immediate physiological needs (survival, soma, maintenance) 2) to enhance the quality of other existing follicles in a quality vs. quantity trade-off or 3) to reduce expenditures on existing reproductive tissues.

Unfortunately, the few studies which have explored the reasons for follicular resorption have demonstrated that evidence exists to support each of these possibilities without necessarily excluding the others. For example, studies that explored the differences in male/female survivorship during starvation have 
shown that the ability to resorb ovarian tissues provides a survival advantage to females but for obvious reasons no survival advantage was afforded to males which suggests that reproductive tissue can support survival directly (Ohgushi, 1996; Osawa, 2005). Other work has shown that resorption directly extends survival and can benefit long-term fecundity by sacrificing short-term fecundity (Boggs and Ross, 1993; Ohgushi, 1996; Osawa, 2005; Richard and Casas, 2009; Rosenheim et al., 2000). On the surface, these examples seem to demonstrate that resorption represents a reversal of resources away from the germ and toward the soma. However, resorption may also be occurring to reduce the metabolic demands made by reproductive tissues. To date neither of these two possibilities have been explicitly tested.

Since in many insects multiple follicles exist simultaneously, the resorption of some inferior follicles to support the remaining follicles is also a possibility. Some evidence for this scenario does exist. In the cockroach, Periplaneta americana, starvation induces resorption of ovarian follicles and a release of intact yolk-proteins back into the hemolymph. Because of the release of intact yolk-proteins the vitellogenin content of the hemolymph increases during starvation despite the fat body (the synthetic site of yolk proteins) remaining inactive (Bell, 1971). The release of intact yolk-proteins suggests that follicles are dismantled in a way that retains certain reproductive resources for reuse in other follicles. However, this example also fails to address the possibility that a reduction of resource expenditures on excess reproductive tissue is the main reason for resorption. 
Better evidence for a scenario whereby some reproductive tissue is sacrificed for the benefit of the remainder comes from $C$. elegans. When apoptosis of oocytes is blocked in this species, average oocyte quality is severely impaired. The reduction of oocyte quality suggests that the pool of resources available to germ cells is limited and that some oocytes must be sacrificed to maintain the ability of those remaining to properly develop (Andux and Ellis, 2010).

Evidence from the cockroach Nauphoeta cinerea shows that the reasons for follicular resorption are not easily teased apart. In this species, starvation causes resorption of the terminal follicle. However, immature secondary follicles which do not represent a significant cache of resources are also resorbed which would impair future reproductive potential should nutritional conditions improve (Barrett et al., 2007). The impairment of future reproductive potential does not clearly conform to either a resorption-for-survival scenario nor one in which recovered resources are being reused for reproduction and may represent the best example of an attempt to reduce the maintenance expenditures on reproductive tissues during nutritional limitation.

Strategies where resources recovered from resorption are returned to the soma or are reused for reproduction are not necessarily mutually exclusive. It is conceivable for each of the aforementioned resorption strategies to occur at the same time or to occur at different times within the same animal in response to different stimuli. It is also possible that the specific class of resources recovered from resorption may be treated in different ways. Since resorption is the only 
known mechanism by which reductions in reproductive output are made (as opposed to a cessation of oogenesis when food is limited), the extent or rate of follicular resorption provides a uniquely observable indicator or window to view the balance of resource allocation between somatic and reproductive physiology. 


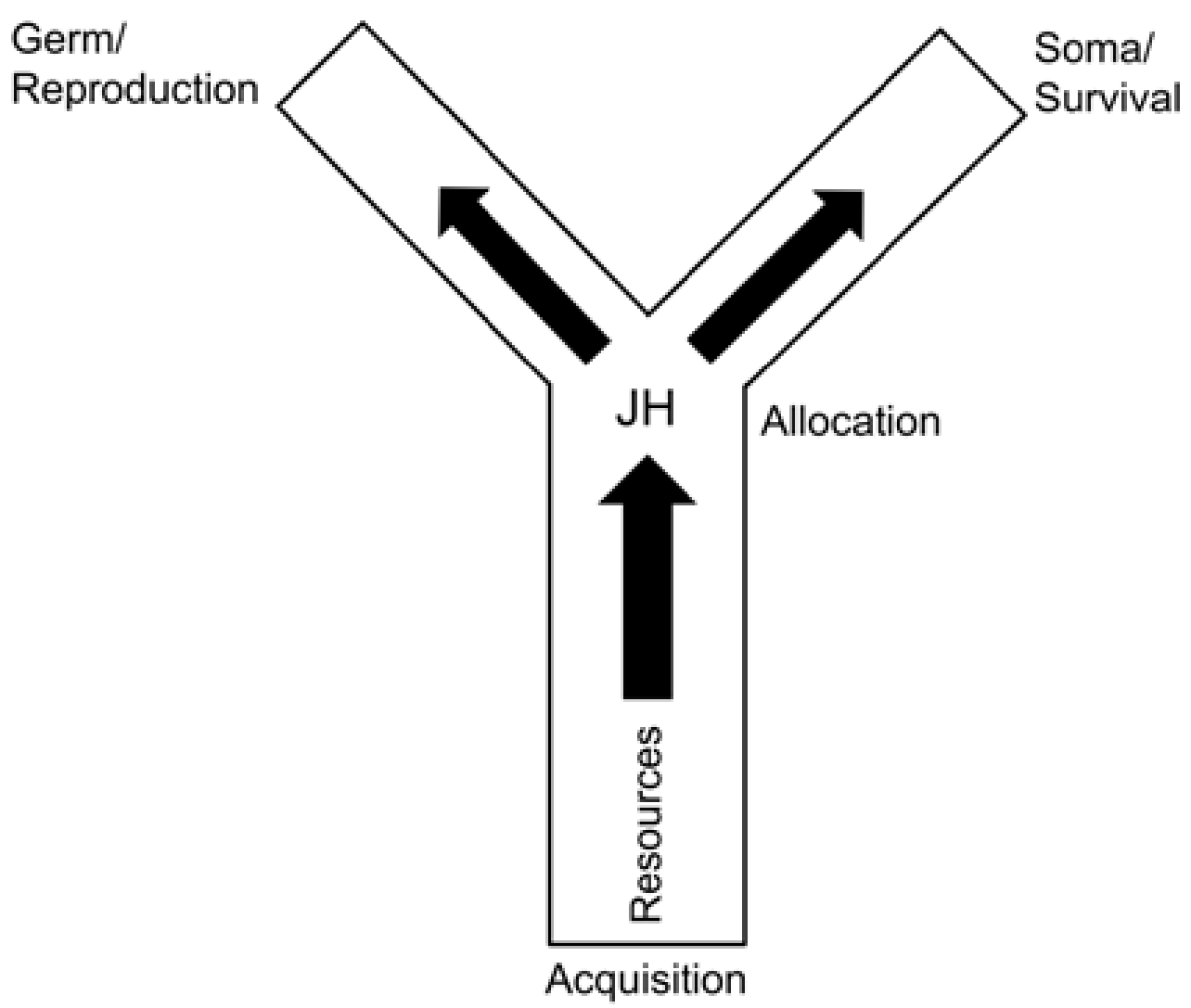

Figure 1: The Y-model of resource allocation. Acquired resources are allocated towards either soma/survival or germ/reproduction physiology. Many life-history and fecundity trade-offs in insects are mediated by $\mathrm{JH}$ in a way that is compatible with the Y-model. Adapted from de Jong and van Noordwijk (1992) and Tatar and Carey (1995). 


\subsection{References}

Almeida, A.S., Andrade Mendronho, R., Ortiz Valencia, L.I. 2009. Spatial analysis of dengue and the socioeconomic context of the city of Rio de Janeiro Southeastern Brazil). Rev. Saúde Pública. 43, 4.

Belles, X., Martin, D., Piulachs, M.-D., 2005. The mevalonate pathway and the synthesis of juvenile hormone in insects. Annual. Review Entomology. 50, 181-199.

Boggs, C.L. 1990. A general model of the role of male-donated nutrients in female insects reproduction. American Naturalist. 136, 598-617.

Boggs, C.L. 2009. Understanding insect life histories and senescence through a resource allocation lens. Functional Ecology. 23.

Borovsky D., Carlson, D.A., Hancock, R.G., Rembold, H., van Handel, E. 1994. De novo biosynthesis of juvenile hormone III and I by the accessory glands of the male mosquito. Insect Biochemistry and Molecular Biology. $24,437-444$.

Briegel, H., 1990. Metabolic relationship between female body size, reserves, and fecundity of Aedes aegypti. Journal of Insect Physiology. 36, 165172.

Briegel, H., Knüsel, I., Timmermann, S.E., 2001. Aedes aegypti: size, reserves, survival, and flight potential. Journal of Vector Ecology. 26, 2131.

Briegel, H., Hefti, M., Di Marco, E. 2002. Lipid metabolism during sequential gonotrophic cycles in large and small females of Aedes aegypti. Journal of Insect Physiology. 48, 547-554.

Carwardine, S.L., Hurd, H., 1997. Effects of Plasmodium yoelii nigeriensis infection on Anopheles stephensi egg development and resorption. Medical and Veterinary Entomology. 11, 265-269.

Caroci, A.S., Li, Y., Noriega, F.G. 2004. Reduced juvenile hormone synthesis in mosquitoes with low teneral reserves reduces ovarian previtellogenic development in Aedes aegypti. Journal of Experimental Biology. 207: 2685-2690.

Clements, A.N., 1992. The Biology of Mosquitoes, Chapman \& Hall, London. 
Clements, A.N., Boocock M.R. 1984. Ovarian development in mosquitoes stages of growth and arrest and follicular resorption. Physiological Entomology. 9, $1-8$.

Chapman, T., Bangham, J., Vinti, G., Seifried, B., Lunch, O.,Wolfner, M.F., Smith, H.K., Partridge, L. 2003. The sex peptide of Drosophila melanogaster: female post-mating responses analyzed by using RNA interference. Proceedings of the National Academies Science. 100: 99239928.

Cheon, H.M., Seo, S.J., Sun, J.X., Sappington, T.W., Raikhel, A.S. 2001. Molecular characterization of the VLDL receptor homolog mediating binding of lipophorin in oocyte of the mosquito Aedes aegypti. Insect Biochemistry and Molecular Biology. 31, 753-60.

Cho, K.H., Raikhel, A.S. 2001. Organization and developmental expression of the mosquito vitellogenin receptor gene. Insect Molecular Biology and Biochemistry. 10, 465-474.

Clancy, D.J., Gems, D. Harshman, L.G. Oldham, S., Stocker, H., Hafen, E., Leevers, S.J., Partridge, L. 2001. Extension of life-span by loss of $\mathrm{CHICO}$, a Drosophila insulin receptor substrate protein. Science. 292, 104-106.

Clements, A.N., Boocock, M.R. 1984. Ovarian development in mosquitoes: stages of growth and arrest and follicular resorption. Physiological Entomology. 9, 1-8.

de Jong, G., van Noordwijk, A.J. 1992. Acquisition and allocation of resources: Genetic (co)variances, selection, and life-histories. The American Naturalist. 4, 749-770.

Dhadialla, T.S., Raikhel, A.S. 1994. Endocrinology of mosquito vitellogenesis. In: Davey, K.G., Peter, R.E., Tobe, S.S. (Eds.), Perspectives in Comparative Endocrinology. National Research Council of Canada. 275-281.

Dingle, H., Winchell, R. 1997. Juvenile hormone as a mediator of plasticity in insect life histories. Archives of Insect Biochemistry and Physiology. $35,359-373$ 
Emlen, D.J., Nijhout, H.F. 1999. Hormonal control of male horn length dimorphism in the horned beetle Onthophagus taurus. Journal of Insect Physiology. 45, 45-53.

Emlen, D.J., Nijhout, H.F. 2001. Hormonal control of male horn length dimorphism in the dung beetle Onthophagus taurus (Coleoptera: Scarabaeidae): a second critical period of sensitivity to juvenile hormone. Journal of Insect Physiology. 47, 1045-1055.

Engelmann, F. 1968. Endocrine control of reproduction in insects. Annual Reviews. 13, 1-26.

Feinsod, F.M., Spielman, A. 1980a. Nutrient-mediated juvenile hormone secretion in mosquitoes. Journal of Insect Physiology. 26, 113-117.

Feinsod, F.M., Spielman, A. 1980b. Independently regulated juvenile hormone activity and vitellogenesis in mosquitoes. Journal of Insect Physiology. 26, 829-832.

Flatt, T., Kawecki, T.J. 2007. Juvenile hormone as a regulator of the trade-off between reproduction and life span in Drosophila melanogaster. Evolution. 61, 1980-1991.

Flatt, T., Tu, M.P. Tatar, M. 2005. Hormonal pleiotropy and the juvenile hormone regulation of Drosophila development and life history. Bioessays. 27, 999-1010.

Foster, W.A. 1995. Mosquito sugar feeding and reproductive energetics. Annual Review of Entomology. 40, 443-474.

Fronstin, R.B., Hatle, J.D. 2008. A cumulative feeding threshold required for vitellogenesis can be obviated with juvenile hormone treatment in lubber grasshoppers. Journal of Experimental Biology. 211, 79-85.

Gilbert, L.I., Granger, N.A., Roe, R.M. 2000. The juvenile hormones: Historical facts and speculations on future research directions. Insect Biochemistry and Molecular Biology. 30, 617-644.

Greenplate, J.T., Glaser, R.L., Hagedorn, H.H. 1985. The role of factors from the head in the regulation of egg development in the mosquito Aedes aegypti. Journal of Insect Physiology. 31, 323-329

Gwadz, R.W. 1969. Regulation of blood meal size in the mosquito. Journal of Insect Physiology. 15, 2039-2044. 
Gwadz, R.W., Spielman, A. 1973. Corpus allatum control of ovarian development in Aedes aegypti. Journal of Insect Physiology. 19, 14411448.

Hagedorn, H.H., Turner, S., Hagedorn, E.A., Pontecorvo, D., Greenbaun, P., Pfeiffer, D., Wheelock, G., Flanagan, T.R. 1977. Post emergence growth of the ovarian follicles of Aedes aegypti. Journal of Insect Physiology. 23, 203-206.

Hansen, I.A., Attardo, G.M., Park, J., Peng, Q., Raikhel, A.S. 2004. Target of rapamycin-mediated amino acid signaling in mosquito anautogeny. Proceedings of the National Academy of Sciences of the United States of America. 101, 10626-10631.

Harshman L.G., Zera, A. 2007. The cost of reproduction: the devil in the details. Trends in Ecology and Evolution. 22, 80-86.

Hernandez-Martinez, S., Mayoral, S.G., Li, Y. Noriega, F.G. 2007. Role of juvenile hormone and allatotropin on nutrient allocation, ovarian development and survivorship in mosquitoes. Journal of Insect Physiology. 53, 230-234.

Jones, G. 1995. Molecular mechanisms of action of juvenile hormone. Annual Review of Entomology. 40, 147-169.

Karlsson, B., 1998. Nuptial gifts, resource budgets and reproductive output in a polyandrous butterfly. Ecology. 79, 2931-2940

Klowden, M.J. 1997. Endocrine aspects of mosquito reproduction. Archives of Insect Biochemistry and Physiology. 35, 491-512.

Kokoza, V.A., Raikhel, A.S., 1997. Ovarian- and somatic-specific transcripts of the mosquito clathrin heavy chain gene generated by alternative 5-exon splicing and polyadenylation. Journal of Biological Chemistry. 272, 11641170.

Lea, A.O. 1963. Some relationships between environment, corpora allata, and egg maturation in Aedine mosquitoes. Journal of Insect Physiology. 9, 793-809.

Li Y., Hernandez-Martinez, S., Unnithan, G.C., Feyereisen, R., Noriega, F.G. 2003. Activity of the corpora allata of adult female Aedes aegypti: effects of mating and feeding. Insect Biochemistry and Molecular Biology. 33, 1307-1315. 
Martin, D., Wang, S.F., Raikhel, A.S. 2001. The vitellogenin gene of the mosquito Aedes aegypti is a direct target of ecdysteroid receptor. Molecular and Cellular Endocrinology. 173, 75-86.

McCall, K. Eggs over easy: cell death in the Drosophila ovary. 2004. Developmental Biology. 274, 3-14.

Miner, A.L., Rosenberg, A. J., and H.F. Nijhout. 2000. Control of growth and differentiation of the wing imaginal disk of Precis coenia (lepidoptera: Nymphalidae). Journal of Insect Physiology. 46, 251-258.

Moczek, A.P. Emlen, D.J. 2000. Male horn dimorphism in the scarab beetle, Onthophagus taurus: do alternative reproductive tactics favour alternative phenotypes? Animal Behavior. 59, 459-466.

Moczek, A.P., Nijhout, H.F. 2004. Trade-offs during the Development of Primary and Secondary Sexual Traits in a Horned Beetle. American Naturalist. 163, 184-191.

Moshitzky, P., Fleischmann, I., Chaimov, N.,Saudan, P., Klauser, S., et al. 1996. Sex peptide activates juvenile hormone biosynthesis in the Drosophila melanogaster corpus allatum. Archives of Insect Physiology and Biochemistry. 32, 363-74.

Nayar, J.K., van Handel, E., 1971. The fuel for sustained mosquito flight. Journal of Insect Physiology. 17, 471-481.

Nijhout, H.F., Emlen, D.J. 1998. Competition among body parts in the development and evolution of insect morphology. Proceedings of the National Academy of Sciences of the United States of America. 95, 3685-3689.

Nijhout, H.F., Williams, C.M. 1974. Control of molting and metamorphosis in the tobacco hornworm Manduca sexta cessation of juvenile hormone secretion as a trigger for pupation. Journal of Experimental Biology. 61, 493-502.

Nijhout, M.M., Riddiford, L.M. 1974. The control of egg maturation by juvenile hormone in the tobacco hornworm moth Manduca sexta. Biological Bulletin. 146, 377-392.

Nijhout, M.M. 1975. A threshold size for metamorphosis in the Tobacco Hornworm (Manduca sexta). Biological Bulletin. 149, 214-225. 
Noriega, F.G., Shah, D.K. Wells, M.A. 1997. Juvenile hormone controls early trypsin gene transcription in the midgut of Aedes aegypti. Insect Molecular Biology. 6, 63-66.

Noriega, F.G. 2004. Nutritional regulation of JH synthesis: a mechanism to control reproductive maturation in mosquitoes? Insect Biochemistry and Molecular Biology. 34, 687-693.

Noriega, F.G., Ribeiro, J.M.C. Koener, J.F. Valenzuela, J.G. HernandezMartinez, S., Pham, V.M., Feyereisen, R. 2006. Comparative genomics of insect juvenile hormone biosynthesis. Insect Biochemistry and Molecular Biology. 36, 366-374.

Raikhel, A.S., Dhadialla, T. 1992. Accumulation of yolk proteins in insect oocytes. Annual Reviews of Entomology. 37, 217-251.

Raikhel, A.S. Lea, A.O. 1985. Hormone-mediated formation of the endocytic complex in mosquito oocytes. General and Comparative Endocrinology. $57,422-433$.

Raikhel, A.S., Lea, A.O. 1991. Control of follicular epithelium development and vitelline envelope formation in the mosquito; role of juvenile hormone and 20-hydroxyecdysone. Tissue \& Cell. 23, 577-591.

Raikhel, A.S., Lea, A.O. 1990. Juvenile hormone controls previtellogenic proliferation of ribosomal RNA in the mosquito fat body. General and Comparative Endocrinology. 77, 423-434.

Rantala, M.J., Vainikka, A., Kortet, R. 2003. The role of juvenile hormone in immune function and pheromone production tradeoffs: a test of the immunocompetence handicap principle. Proceedings of the Royal Society of London Series B. 270, 2257-2261.

Riddiford, L.M. 1980. Insect Endocrinology: Action of Hormones at the Cellular Level. Annual Reviews. 42, 511-528.

Riddiford, L.M. 1996. Juvenile hormone: The status of its "Status quo" Action. Archives of Insect Biochemistry and Physiology. 32, 271-286.

Röller, H., Dahm, K.H., Sweeley, C.C., Trost, B.M. 1967. The structure of the juvenile hormones. Angew. Chemie. 4, 190-191. 
Seo, S.J., Cheon, H.M., Sun, J. Sappington, T.W., Raikhel. A.S. 2003. Tissueand stage-specific expression of two lipophorin receptor variants with seven and eight ligand- binding repeats in the adult mosquito. Journal of Biological Chemistry. 278, 41954-41962.

Shin-Hong, S., Hansen, I.A., Zhu, J., Sieglaff, D., Raikhel, A.S. 2008. Juvenile hormone connects larval nutrition with target of rapamycin signaling in the mosquito Aedes aegypti. Journal of Insect Physiology. 54, 231-239.

Sláma, K., Williams, C.M. 1965. Juvenile hormone activity for the bug Pyrrhocoris apterus. Proceedings of the National Academies of Science. $54,411-414$.

Socha, R., Sula, J. 2007. Differential allocation of protein resources to flight muscles and reproductive organs in the flightless wing-polymorphic bug, Pyrrhocoris apterus (L.) (Heteroptera). Journal of Comparative Physiology. B, Biochemical Systemic and Environmental Physiology. 178, 179-188.

Strambi, A., Strambi, C., Cayre, M. 1997. Hormonal control of reproduction and reproductive behavior in crickets. Archives of Insect Biochemistry and Physiology. 35, 393-404.

Tatar, M., J.R., Carey 1995. Nutrition mediates reproductive trade-offs with age specific mortality in the beetle Callosobruchus maculatus. Ecology. 76, 2066-73

Tatar, M., Kopelman, A., Epstein, D., Tu, M.-P., Yin, C.-M. Garofalo, R. S. 2001 A mutant Drosophila insulin receptor homolog that extends life- span and impairs neuroendocrine function. Science. 292, 107-110.

Telang, A., Wells, M.A. 2004. The effect of larval and adult nutrition on successful autogenous egg production by a mosquito. Journal of Insect Physiology. 50, 677-685.

Trowell, S.C. 1992. High affinity juvenile hormone carrier proteins in the hemolymph of insects. Comparative Biochemistry and Physiology 103B, 795-808.

Truman, J.W., Riddiford, L.M. 2002. Endocrine insights into the evolution of metamorphosis in insects. Annual Reviews. 47, 467-500. 
Truman, J.W., Hiruma, K., Allee, J.P. 2006. Juvenile hormone is required to couple imaginal disc formation with nutrition in insects. Science. 312, 1385-1388.

Tu, M.P., Flatt, T., Tatar, M. 2006. Juvenile and steroid hormones in Drosophila melanogaster longevity. In E. J. Masoro and S. N. Austad, eds. Handbook of the biology of aging, 6th ed. Academic Press San Diego, CA. $415-448$.

Tu, M.P., Yin, C.M., Tatar, M. 2005. Mutations in insulin signaling pathway alter juvenile hormone synthesis in Drosophila melanogaster. General and Comparative Endocrinology. 142, 347-356.

Uchida, K., Nishizuka, M., Ohmori, D., Ueno, T., Eshita, Y., Fukunaga, A. 2004. Follicular epithelial cell apoptosis of atretic follicles within developing ovaries of the mosquito Culex pipiens pallens. Journal of Insect Physiology. 50, 903-912.

Van Handel, E. 1993. Fuel metabolism of the mosquito (Culex quinquefasciatus) embryo. Journal of Insect Physiology. 39, 831-33.

Van Noordwijk, A.J., de Jong, G., 1986. Acquisition and allocation of resources: their influence on variation in life history tactics. American Naturalist. 128, 137-142.

Wigby, S., Chapman, T. 2005. Sex peptide causes mating costs in female Drosophila melanogaster. Current Biology. 15, 316-321.

Wigglesworth, V.B. 1934. The physiology of ecdysis in Rhodnius prolixus. 11. Factors controlling moulting and metamorphosis. Quarterly Journal of Microscopic Science. 77, 191-222.

Wigglesworth, V.B. 1970. Insect Hormones. W.H. Freeman and Company. San Francisco.

Williams, C.M. 1961. The juvenile hormone II: its role in the endocrine control of molting, pupation, and adult development in the cecropia silkworm. Biological Bulletins. 121, 572-585.

Zhou, G., Pennington, J.E., Wells, M.A., 2004. Utilization of pre-existing energy stores of female Aedes aegypti mosquitoes during the first gonotrophic cycle. Insect Biochemistry and Molecular Biology 34, 919-25. 
Ziegler, R., Ibrahim, M.M. 2001. Formation of lipid reserves in fat body and eggs of the yellow fever mosquito, Aedes aegypti. Journal Insect Physiology $47,623-27$.

Zera, A.J., Cisper, G. 2001. Genetic and diurnal variation in the juvenile hormone titer in a wing-polymorphic cricket: implications for the evolution of life histories and dispersal. Papers in the Biological Sciences. 293-306. 


\section{Chapter 3: Nutrient limitation results in juvenile hormone-mediated resorption of previtellogenic ovarian follicles in mosquitoes}

\subsection{Abstract}

Juvenile hormone $(\mathrm{JH})$ is a central hormonal regulator of previtellogenic development in female Aedes aegypti mosquitoes. Juvenile hormone levels are low at eclosion and increase during the first day after adult emergence. This initial rise in $\mathrm{JH}$ is essential for female reproductive maturation. After previtellogenic maturation is complete, the mosquito enters a 'state-of-arrest' during which $\mathrm{JH}$ synthesis continues at a slower pace and further ovary development is repressed until a blood meal is consumed. By examining the relationships between juvenile hormone, follicular resorption and nutrition in A. aegypti, we were able to define a critical role of $\mathrm{JH}$ during the previtellogenic resting stage. The rate of follicular resorption in resting stage mosquitoes is dependent on nutritional quality. Consumption of water alone caused the rate of follicular resorption to reach over $20 \%$ by day 7 after emergence. Conversely, feeding a $20 \%$ sucrose solution caused resorption to remain below $5 \%$ during the entire experimental period. Mosquitoes fed a $3 \%$ sucrose solution showed rates of resorption intermediate between water and $20 \%$ sucrose and only reached $10 \%$ by day 7 after emergence. Follicular resorption is related to $\mathrm{JH}$ levels. Ligated abdomens separated from a source of $\mathrm{JH}$ (the corpora allata) showed an increase in resorption comparable to similarly aged starved mosquitoes $(16 \%)$. Resorption in ligated abdomens was reduced to $6 \%$ by application of methoprene. The application of methoprene was also sufficient to prevent 
resorption in intact mosquitoes starved for 48 hours (14\% starved vs. $4 \%$ starved with methoprene). Additionally, active caspases were localized to resorbing follicles indicating that an apoptotic cell-death mechanism is responsible for follicular resorption during the previtellogenic resting stage. Taken together, these results indicate that $\mathrm{JH}$ mediates reproductive trade-offs in resting stage mosquitoes in response to nutrition.

\subsection{Introduction}

The appropriate allocation of nutrients between competing demands such as reproduction, growth, maturation or flight is a critical component of an insect's life-history strategy (Boggs, 1992; Stevens et al., 2000; Wheeler 1996). Insects must not only allocate nutrients properly within each developmental stage, but must also consider the effects of immediate resource allocations on future reproduction and overall fitness (Boggs, 1981; Pianka and Parker, 1975; Rivero et al., 2001). Any over-allocation of resources to one specific activity will have adverse effects on other physiological activities. These life-history and resource allocation trade-offs can be best uncovered with examples of competition between nutrient intensive processes such as flight and reproduction (flightoogenesis syndrome) (Oliveira et al., 2006), sexual advertisement and immunity (immuno-competence handicap hypothesis) (Rantala et al., 2003), nutrition and adult phenotype (horned beetle polyphenism) (Moczek, 1998; Moczek and Nijhout, 2004) or with examples where nutrient limitation forces developmental, reproductive or other nutrient allocation decisions to be made (Nijhout and Wheeler, 1982; Noriega, 2004; Wheeler 1996). 
Reproductive trade-offs in adult insects occur primarily through the process of oosorption. By resorbing excess reproductive tissues, insects can alter previous reproductive decisions by redirecting resources away from reproduction in favor of competing physiological activities (Boggs and Ross, 1993; Ohgushi, 1996; Osawa, 2005). The redirection of reproductive nutrients to support alternative processes is supported by studies that explored the differences in female/male survivorship under starvation conditions which have demonstrated that the resources contained within the ovary can be a valuable nutrient store for females (and one unavailable to males) when dealing with environmental constraints (Ohgushi, 1996; Osawa, 2005). Other work has suggested that the nutritional resources recovered from the ovary can be used to improve survival, extend search time for resources or hosts, and maximize overall fitness (Boggs and Ross, 1993; Ohgushi, 1996; Osawa, 2005; Richard and Casas, 2009; Rosenheim et al., 2000). Like many resource allocation decisions, the balance between nutrition, reproduction, and survival is likely to be mediated hormonally (Flatt et al., 2005; Trumbo and Robinson, 2004)

Juvenile hormone $(\mathrm{JH})$ has been suggested as the main hormonal factor that controls follicular resorption in some insects, a role that conveniently meshes with $\mathrm{JH}$ 's broader participation in mediating resource allocation trade-offs (Reviewed in Bell and Bohm, 1975). In many insects, yolk protein production is dependent on $\mathrm{JH}$ synthesis and overall nutritional status. Nutritional limitation often leads to cessation of $\mathrm{JH}$ synthesis (Caroci et al., 2004), a reduction of yolk protein synthesis and increased oosorption (Barrett et al., 2008; Bell and Bohm, 
1975). The developmental pauses, unique pattern of hormonal secretions and discrete feeding habits of anautogenic mosquitoes have made a regulatory scheme based on nutrition production and $\mathrm{JH}$ synthesis more difficult to define. Thus, oosorption in mosquitoes has only been well-described after a blood meal (Carwardine and Hurd 1997; Clements and Boocock, 1984; Uchida et al., 2004); the highest rates of oosorption are seen during this time when up to $27 \%$ of follicles will become resorbed despite advanced vitellogenic development (Clements and Boocock, 1984). Follicular resorption after a blood meal can be greatly increased by Plasmodium infection in Anopheline spp. mosquitoes (Ahmed and Hurd, 2006; Carwardine and Hurd 1997; Hopwood et al., 2001).

Studies exploring the relationship between sugar feeding and reproduction in mosquitoes have clearly demonstrated that sugar feeding improves fecundity in some circumstances but have failed to provide a mechanistic or hormonal reason for the observed alterations in reproductive allocations. (Nayar and Sauerman, 1971; Briegel, 1990; Naksathit et al., 1999; Foster, 1995). In adult $A$. aegypti, JH's most clearly defined role is as the hormone responsible for regulating previtellogenic reproductive development. A role for $\mathrm{JH}$ in coordinating nutrition and reproduction by regulating the resorption of follicles before a blood meal has not been described in any mosquito.

Descriptions of apoptosis and oosorption have rarely been described in mosquitoes. Reports by Hopwood et al. (2001), Ahmed and Hurd (2006), and Uchida et al. (2004) have all attempted to clarify the progression of resorption following a blood meal. The description by Uchida et al. (2004) identifies active 
caspases solely in the follicular epithelium. Hopwood et al. (2001) and Ahmed and Hurd (2006) have described markers of apoptosis occurring in patches of the follicular epithelium as preceding resorption of the follicle in Plasmodium-infected mosquitoes. From these observations, a regulatory role for the follicular epithelium in resorption has been surmised. It is not clear if resorption, in sugar fed $A$. aegypti, also occurs through apoptotic mechanisms and if follicular resorption is mediated by the follicular epithelium.

In this study, we specifically investigated the relationships between nutrition, juvenile hormone and reproduction in $A$. aegypti during the previtellogenic resting stage by exploring three main questions: 1) Does nutrition during the resting stage affect follicular resorption and overall fecundity? 2) Does JH during the previtellogenic resting stage prevent follicular resorption? And finally, 3) Which cell-death mechanisms are responsible for follicular resorption in a previtellogenic mosquito? Each of these interrelated factors is an important component of the fitness and life-history strategy of A. aegypti which also makes them important for understanding the relationship between this disease vector and its environment. The conspicuous and observable nature of the insect ovary in conjunction with its close dependence on hormonal and nutritional status makes oosorption an ideal experimental mechanism for exploring resource and reproductive trade-offs. By answering these questions it is clear that $\mathrm{JH}$ synthesis during the resting stage participates in the regulation of fecundity by preventing apoptosis and resorption in previtellogenic follicles. 
Furthermore, the resorption of follicles is dependant, at least in part, on the quality of nutrition during the resting stage.

\subsection{Methods}

\subsubsection{Insects}

A colony of Aedes aegypti of the Rockefeller strain was maintained at $28^{\circ} \mathrm{C}$ with $80 \%$ relative humidity under a 16 hour day- 8 hour night regime. Males and females emerging during the same 15 hour overnight period were collected and transferred to a screened container. Mated adults were offered a cotton pad soaked in $3 \%$ sucrose solution.

\subsubsection{Observations of oosorption in previtellogenic follicles}

Adult mosquitoes were fed $3 \%$ sucrose from eclosion until 2 days after emergence to allow normal previtellogenic follicular maturation. After the second day post- emergence, mosquitoes were transferred to one of three nutritional regimes: 1) $20 \%$ sucrose 2 ) water only (starved) or 3 ) continued on $3 \%$ sucrose. Ten female mosquitoes were collected each day for 5 days for each nutritional regime beginning on day 3 , post-emergence. Mosquitoes within each feeding regime were collected for at least three independent replicates. All mosquitoes were anesthetized by chilling for $5-10$ minutes at $4^{\circ} \mathrm{C}$ prior to dissection. Ovaries from these mosquitoes were removed, rinsed in Aedes physiological saline (APS)( $\mathrm{MgCl}_{2}$ 0.6mM; KCl 4.0mM NaHCO 3 1.8mM; NaCl 150.0 mM; HEPES 25.0 $\mathrm{mM} ; \mathrm{CaCl}_{2} 1.7 \mathrm{mM}$ ) and stained with $0.5 \%$ neutral red solution in acetate buffer at pH 5.2 (Sigma Aldrich, St. Louis, MO) for 10 seconds to visualize resorbing follicles. Neutral red stains the lysosomes associated with resorbing follicles and 
can clearly indicate follicle status (Winckler, 1974; Bell and Bohm, 1975; Clements and Boocock, 1984; Hopwood et al., 2001). The ovaries were rinsed a second time in APS and placed under a coverslip. Photographs were taken of the previtellogenic ovaries using a DM 5500 B Leica fluorescence microscope, a Leica DFC 310 FX mounted camera and Leica LAS imaging software. Ovaries were later scored using Leica LAS imaging software for total follicle count and also for the presence of any resorbing follicles.

\subsubsection{Abdominal ligations}

Fifteen female mosquitoes were randomly selected from a mixed-gender population fed $3 \%$ sucrose until 3 days old. After being cold anesthetized for 5-10 minutes a fine Kevlar thread (Stren Powerbraid, Wilmington, DE) was firmly tied around the mosquito anterior to abdominal segment I and posterior to the halters (Figure 2). The abdomen was removed and the anterior end sealed with wax (Boekel tackiwax, Philadelphia, PA). The loose end of the tied Kevlar thread was hung from a strip of parafilm (Pechiney Plastic Packaging; Chicago, Illinois) and

placed inside of a humid glass chamber $\left(\sim 700 \mathrm{~cm}^{3}\right)($ Figure 2). The hanging abdomens were treated with either $500 \mathrm{ng}$ of the juvenile hormone analogue Methoprene (Zoecon Co., Palo Alto, CA) in $0.5 \mu \mathrm{l}$ acetone or $0.5 \mu \mathrm{l}$ of acetone alone. After 48 hours, viable abdomens were dissected, and the ovaries rinsed with APS. The ovaries were stained with neutral red for 10 seconds, rinsed again and placed under a coverslip. Four independent replicates were conducted. Photographs were taken of the previtellogenic ovaries and scored as previously described. 


\subsubsection{Hormonal manipulations of intact mosquitoes}

At 3 days post-emergence, randomly selected mosquitoes from a mixed gender population were cold anesthetized and topically treated with either $500 \mathrm{ng}$ of methoprene dissolved in $0.5 \mu \mathrm{l}$ acetone or with $0.5 \mu \mathrm{l}$ acetone alone. Insects were then transferred to a water-only diet for 2 days before being dissected and assayed with neutral red, photographed and scored as previously described. Two independent replicates were conducted.

\subsubsection{Active caspase in-situ assay}

Active caspases were detected in the ovaries of previtellogenic mosquitoes using a sulforhodamine multi-caspase activity kit (AK-115, Enzo Life Sciences, Plymouth Meeting, PA). In this kit, sulforhodamine labeled valylalanylaspartic acid fluoromethyl ketone (SR-VAD-FMK) enters the cell and acts as a specific inhibitor of apoptosis by covalently binding to the reactive cysteine residue indicative of an active caspase. The sulforhodamine label allows detection and localization of active caspases by fluorescence microscopy. Ovaries were removed from 4 day old previtellogenic mosquitoes that were fed $3 \%$ sucrose, rinsed in APS, and incubated in the $1 / 3 \mathrm{X}$ reaction medium $(10 \mu \mathrm{l}$ of 30x SR-VAD-FMK in $290 \mu \mathrm{l}$ of PBS) for 1.5 hours at $26^{\circ} \mathrm{C}$. The labeled ovaries were washed first for 6 hours at $26^{\circ} \mathrm{C}$ and then overnight in $1 \mathrm{X}$ wash solution at $4^{\circ} \mathrm{C}$, rinsed with $1 \mathrm{X}$ PBS and placed under a coverslip. Photographs were taken of the previtellogenic ovaries as described. Visible light and florescence images taken with a Leica Texas red filter set were merged. 


\subsection{Results}

\subsubsection{Nutrition affects previtellogenic follicular resorption}

The nutritional factors that affect follicular resorption were explored by rearing adult mosquitoes under three nutritional regimes: 1) water alone 2) $3 \%$ sucrose in water or 3) $20 \%$ sucrose in water. Follicles were scored as resorbing by possessing one of the two following indicators: 1) Condensed chromatin (pyknotic nuclei) in nurse cells in conjunction with intense staining of cytoplasmic regions of nurse cells or oocyte with neutral red (early resorbing) (Figure 3B) or 2) Intense staining of follicular epithelial cells with neutral red combined with absence of identifiable interior cells types (late resorbing) (Figure 4A). Follicles not conforming to either of the two criteria were not counted as resorbing. The rate of resorption increases daily in mosquitoes fed $3 \%$ sucrose reaching a rate of nearly $10 \%$ on day 7 (Figure 4B). The increase in the number of resorbing follicles was inversely proportional to the decrease in JH synthesis (Figure 4B).

Resorbing follicles at day 3 already comprised between $3 \%$ and $7 \%$ of the total follicles in mosquitoes fed either $3 \%$ sucrose, $20 \%$ sucrose or water alone, indicating that the process of follicular resorption begins prior to day 3 and may be concurrent with previtellogenic development (Figures 4 and 5). An even steeper increase in resorption is seen in mosquitoes fed water only. By day 7 over $20 \%$ of the follicles are resorbing in starved mosquitoes. The increase in resorption seen in mosquitoes fed water is significantly greater than mosquitoes fed $3 \%$ and $20 \%$ sucrose on every day examined (Figures 4 and 5 ). In conjunction with the overall upward trend in starved mosquitoes, this result 
indicates that an increase in the rate of resorption begins quickly under starvation conditions.

The rate of follicular resorption in mosquitoes fed $20 \%$ sucrose declines slowly until day 5 . On days 6 and 7 the rate of follicular resorption begins increasing again but always remains below the rate of resorption for either $3 \%$ sucrose fed or water only mosquitoes (Figures 4 and 5 ). By day 7 only $5 \%$ of follicles are resorbing; a rate that is only half of the rate found in mosquitoes fed $3 \%$ sucrose.

\subsubsection{Methoprene prevents follicular resorption in ligated abdomens}

To establish that juvenile hormone can prevent follicular resorption, abdomens from 3 day old mosquitoes were ligated, treated with $500 \mathrm{ng}$ of methoprene or acetone and incubated for 48 hours. Abdominal ligation is a wellestablished experimental technique to test the effect of $\mathrm{JH}$ deprivation on abdominal tissues (Noriega et al., 1997). By isolating the ovaries from the corpora allata, their endogenous source of juvenile hormone was removed and the rate of follicular resorption increased to over $16 \%$ (Figure 6), a level only seen in intact but starved mosquitoes (Figure 5). Topical application of $500 \mathrm{ng}$ of methoprene was sufficient to prevent the increase in follicular resorption seen in ligated abdomens and limit the rate of follicular resorption to 6\% (Figure 6), a level comparable to 5 day old intact mosquitoes (Figure 5 ).

\subsubsection{Methoprene prevents follicular resorption in starved mosquitoes}

To investigate the connection between nutritional status, hormonal levels and follicular resorption in intact animals, we treated mosquitoes with either 500 
ng of methoprene or acetone alone. These mosquitoes were subsequently starved (fed water only) for two days and assayed as previously described. By applying hormone to starved mosquitoes, we were able to prevent follicular resorption and alter the normal resource allocation response to starvation. Mosquitoes treated with acetone alone and starved for 48 hours showed levels of follicular resorption (14\%) (Figure 7 ) comparable to similarly aged (5 days old) starved mosquitoes (Figure 5). Mosquitoes treated with methoprene showed only a $3.4 \%$ rate of resorption (Figure 7 ). This was comparable to similarly aged mosquitoes fed $20 \%$ sucrose (Figure 5 ).

\subsubsection{Active caspase in-situ assay}

An active caspase in-situ assay was conducted on ovaries from 4 day-old mosquitoes to explore the cell-death mechanisms of follicular resorption in $A$. aegypti. Follicles undergoing resorption contained active caspases as indicated by the sequestration and concentration of a sulforhodamine-labeled caspase inhibitor probe (SR-VAD-FMK) (Figure 8A). Those follicles that were not undergoing resorption in the same ovary did not sequester the probe (Figure 8A).

In follicles that still contained identifiable cellular structures, the intracellular spaces between cell types generally contained the most intense caspase activity (Figure 8B). As interior cellular structures became less welldefined, caspase localization became more diffuse throughout the interior of the follicle (Figure $8 \mathrm{C}$ ). Follicles that no longer contained identifiable internal structures had active caspases localized throughout the follicle but most intensely in the remains of the follicular epithelium (Figure 8D). The pattern of 
caspase localization in resorbing follicles matches closely the pattern of neutral red staining. Resorption appears to begin first within the follicle followed by the follicular epithelium. The follicular epithelium in the final stages of resorption had lost integrity as a covering and also contained the most intense caspase signal. At this late stage, the interior of these follicles contained no remaining cellular structures and did not contain active caspases (not shown).

\subsection{Discussion}

\subsubsection{Nutrition, JH synthesis, and follicular resorption are linked}

In $A$. aegypti, the nutritional regulation of $\mathrm{JH}$ synthesis has been described as a mechanism to control reproductive maturation (Noriega, 2004). Immediately following emergence, a peak of $\mathrm{JH}$ synthesis (nearly $35 \mathrm{fmol} / \mathrm{CA}$ pair/hour) directs the maturation of the ovary and other tissues to support an adult phenotype capable of blood-feeding and reproduction (Caroci et al., 2004; Li et al., 2003; Noriega, 2004; Raikhel and Lea, 1984; Raikhel and Lea, 1991). After 60 hours, maximum previtellogenic development is reached and the mosquito enters a developmental resting stage that feeds exclusively on sugar or nectar until a host is found (Clements, 1992; Hagedorn et al., 1977). From day 3 until day 7 post-emergence, $\mathrm{JH}$ synthesis declines from $15 \mathrm{fmol} /$ pair $\mathrm{CA} /$ hour to 5 fmol/pair CA/ hour (Li et al., 2003) while follicular resorption increases from $3 \%$ of total follicles per female to nearly $10 \%$ per female during the same period in mosquitoes fed $3 \%$ sucrose (Figure 4). Results from hormonal manipulations in isolated abdomens confirmed that the increase in follicle resorption during the resting stage is probably the result of falling $\mathrm{JH}$ titers. This hypothesis was further 
validated since methoprene was able to prevent follicular resorption in intact but starved mosquitoes. Taken together, these results provide strong evidence that follicular resorption is under the control of juvenile hormone and ultimately, nutrition during the resting stage.

Reproduction and nutrition are closely connected through $\mathrm{JH}$ titers. In many insects, starvation or other nutritional limitation leads to reduced $\mathrm{JH}$ synthesis, which in turn, leads to resorption, arrest or other reproductive tradeoffs (Schal et al., 1993; Tobe and Chapman, 1979; Trumbo and Robinson, 2004; Yin et al., 1999). The connection of reproduction with nutrition through $\mathrm{JH}$ synthesis is also true in $A$. aegypti, albeit this relationship has only been previously demonstrated immediately following emergence. Hormonal and nutritional manipulations have shown that females emerging with low teneral reserves will fail to develop ovaries properly (Caroci et al., 2004; HernandezMartinez et al., 2007; Feinsod and Spielman, 1980). A clear function for JH during the resting stage in previtellogenic mosquitoes has, to our knowledge, been previously overlooked (Hagedorn et al., 1977; Lea, 1963; Raikhel and Lea, 1984; Raikhel and Lea, 1991).

The experiments described here indicate that $\mathrm{JH}$ synthesis during the resting stage is an integral component of the allocation and reproductive decisions made as part of the life-history strategy of $A$. aegypti. These results are generally in agreement with data obtained by Söller et al., (1999) that showed methoprene application reduces the apoptosis of nurse cells in the follicles of Drosophila melanogaster and others who have shown the hormone-dependent 
nature of follicular resorption (Bell and Bohm, 1975). The hormonal basis for follicular resorption may not depend on $\mathrm{JH}$ alone. Work with $D$. melanogaster showed starvation-induced apoptosis of follicular cells to be caused by an increased ecdysteroid titer and methoprene prevented this apoptosis by reducing ecdysteroid titers; a result which appears contradictory to observations made in mosquitoes (Terashima et al., 2005). In A. aegypti, ecdysteroid titers are very low prior to a blood meal and are not reduced further by methoprene application at that time (Borovsky et al., 1986). However, many differences exist in the way that $\mathrm{JH}$ and ecdysteroid signaling pathways interact, making comparisons between species difficult (reviewed in Spindler et al., 2009).

Work that explored the interaction between methoprene, ecdysteriods and gene expression changes during midgut remodeling and metamorphosis in $A$. aegypti has shown that methoprene blocks apoptosis in midgut cells by modulating the expression of ultraspiracle A (Usp A) and ecdysone receptor B (Ecr B) as well as genes important to apoptosis (Wu et al., 2006). Later work showed that transcription of AeDronc (an initiator caspase and key component of apoptosis) as well as overall caspase activity were both increased by ecdysone treatment in larval mosquitoes (Cooper et al., 2007). In the adult mosquito ovary, ecdysone treatment caused a nearly 3 -fold increase in AeDronc transcription (Cooper et al., 2007). It is not yet clear if ecdysone increases apoptosis and follicular resorption in previtellogenic ovaries of $A$. aegypti but we cannot reject the idea that the balance of the two hormones may be an important factor in determining the fate of the follicles (Terashima and Bownes, 2004). 


\subsubsection{Follicular resorption as a resource allocation decision}

Previous studies on the effects of sugar feeding on reproduction in $A$. aegypti have shown that sugar can improve fecundity under some circumstances (reviewed in Foster, 1995); although the mechanism for an improvement (or reduction) in fecundity was not elucidated in previous studies. Our results demonstrate that sugar-feeding can prevent follicular resorption during the previtellogenic resting stage. By manipulating the feeding regime in resting stage mosquitoes, we were able to show that the rate of follicular resorption increased as the quality of the sugar meal decreased.

A high sucrose meal was sufficient to prevent a reduction in fecundity as there were significantly more follicles remaining at the end of 7 days in those fed the higher concentration food ( 148.5 follicles vs. 139.8 follicles; $P<.05 ; n=60$; $\mathrm{df}=58$; t-test). These results clearly demonstrate that reproductive trade-offs are made specifically in response to feeding on carbohydrates. More specifically these results demonstrate that sugar feeding can prevent follicular resorption and enhance fecundity.

While we did not explore how the nutrients from resorbed follicles were metabolically allocated, other studies exploring starvation may help explain how these nutrients are reused. Sugar feeding in mosquitoes primarily supports flight activity and lipid synthesis. The majority of lipids (teneral and newly synthesized) are stored in the fat body and are exclusively used to provision eggs after the first blood meal (Briegel, 1990; Briegel et al., 2002; Foster, 1995; Zeigler and Ibrahim, 2001; Zhou et al., 2004). The synthesis of lipids from sugar feeding has been 
reported many times and generally occurs whenever excess carbohydrates are available (Briegel, 1990; Briegel et al., 2001; Foster, 1995; Nayar and Van Handel, 1971). Briegel (1990) found that starvation leads to drastic reductions in whole body lipid and carbohydrate content and much smaller reductions in protein content. Since lipids are primarily stored in the fat body and very little are stored or synthesized in the ovary of previtellogenic mosquitoes (Troy et al., 1975; Zeigler and Van Antwerpen, 2006; Zeigler and Ibrahim, 2001), it does not seem likely that during starvation ovaries are resorbed exclusively for their lipid content. The lack of a clear relationship between resorption and lipid metabolism is further supported by observations demonstrating a limited role for lipids in supporting flight activities (Briegel et al., 2002). If lipids cannot be used for anything but egg provisioning and sugar feeding only supports flight and/or lipid synthesis, then it is hard to see the immediate linkage between sugar feeding and reproduction. Briegel et al., (2001) found anything above a $0.5 \%$ sucrose solution adequate to extend survivorship and stimulate reserve lipid synthesis in nutrient-deficient mosquitoes. When we fed mosquitoes $3 \%$ sucrose, resorption increased on each day examined until reaching nearly $10 \%$ on day 7 (Figure 4). This indicates that $3 \%$ sucrose (or even $20 \%$ sucrose), while potentially adequate to stimulate synthesis of lipids is not adequate to stop resorption and further suggests that resorption in $A$. aegypti is not occurring because of a shortage of lipids and/or carbohydrates.

Another possible reason for follicular resorption in previtellogenic mosquitoes may be the result of a nitrogen/protein limitation. Support for a 
protein-limitation scenario can be found in a report by Judson and DeLumen (1976), who described that treatment of mosquitoes with synthetic analogues of $\mathrm{JH}$ (Cecropia hormone and methoprene) causes increases in ovarian protein content and an increase in overall ovarian size as compared to controls. They did not report the specific nature of the protein increase nor did they explore any effect of juvenoids or juvenile hormone analogues on resorption. An increase in oocyte and follicle size after methoprene application has been subsequently confirmed in our lab (unpublished results). Conversely, starvation until death was found to reduce whole body protein levels by $14 \%$. Although it is unknown what proportion of this protein reduction belongs to the ovaries, it is possible that part of the reduction in protein content is a consequence of resorption (Briegel, 1990). Taken together with our results indicating a role for $\mathrm{JH}$ in preventing resorption, it is conceivable that $\mathrm{JH}$ mediates resource allocations between soma and reproduction during the previtellogenic resting stage in response to protein deficiency. Therefore, it is possible that ovarian follicles are resorbed exclusively for their protein content and this allocation is mediated by juvenile hormone.

Further support for this possibility comes from a basic examination of the life-history strategy of $A$. aegypti. The majority of proteins/nitrogen must be carried over from larval feeding as sugar feeding mosquitoes have limited access to nitrogenous compounds. Qualitative nutritional deficiencies (especially protein) have been reported before as a cause of resorption (Bell and Bohm, 1975). Although the synthesis of some non-essential amino acids does occur from 
sugars, it is not clear how sugar feeding would dictate protein and reproductive allocations (Zhou et al., 2004).

\subsubsection{Follicular resorption as a life-history strategy}

The resorption of follicles represents a reversal of nutrients away from reproduction and towards alternative activities and reflects the need to balance present and future reproduction to maximize fitness (Boggs and Ross, 1993; Ohgushi, 1996; Rosenheim et al., 2000). Studies exploring reproductive tradeoffs and life-history strategies may offer some clues to explain the reasons for the follicular resorption we observed in previtellogenic mosquitoes. In the nectivorous butterfly, Speyeria mormonia, nutritional limitation causes reductions in fecundity via follicular resorption but does not cause reductions to life span, suggesting survival needs get "first claim" on any resources (Boggs and Ross, 1993). Studies exploring oosorption and survival in the lady beetle, Epilachna niponica, found that females were able to tolerate periods of starvation better than males because of their ability to resorb follicles and may realize significant future benefits to fitness by resorbing eggs in the present (Ohgushi, 1996). Theoretical models using the wasp, Aphytis aonidiae, demonstrated that the resorption of follicles only made sense fitness-wise if it increased life-span and more specifically, increased host-seeking time (Rosenheim et al., 2000). The translation of reproduction into flight (i.e., host-seeking time) is supported by observations in the blood-sucking bug, Rhodnius prolixus. Fecundity was significantly reduced in bugs subjected to daily exhaustive flight as a result of increased lipid oxidation (Oliveira et al., 2006). However, in Aedine mosquitoes, 
lipids are generally not used to support flight (Briegel et al., 2002; Nayar and Handel, 1971). Despite this, flight and host-seeking are metabolically costly in the mosquito and the resorption of follicles to support these costs might make sense from a life-history strategy viewpoint even if the biochemical nature of this trade-off is not immediately obvious (Foster, 1995).

Another explanation for the pattern of follicular resorption we observed in A. aegypti may be better illustrated by work that examined the adaptive and nutritional value of resorption in the parasitoid wasp, Eupelmus vuilleti. In this species, the resorption of a single egg is only able to cover less than $10 \%$ of daily energy needs (Casas et al., 2005). In E. vuilleti as well as the mosquito, a simple relationship between nutrition and resorption is not clearly evident as resorbed eggs do not seem to be able to provide the kinds of benefits to survival that one would expect (Richard and Casas, 2009). Richard and Casas (2009) explain resorption as a buffer against stochasticity in the environment whereby a low value resource (ovarian follicles) can confer a fitness advantage primarily because of the controllability of this resource. While many of the observations stated here have not been specifically described in mosquitoes, they do demonstrate that additional layers of decision-making are likely to exist which need to be considered when explaining resorption in mosquitoes.

\subsubsection{Progression of follicular resorption in previtellogenic mosquitoes}

The progression of resorption in previtellogenic follicles supports the assertion by Uchida et al. (2004) that the follicular epithelium participates in the regulation of resorption in mosquitoes. Additional observations about the 
progression of resorption and localization of caspases in mosquito follicles were possible in this study because of a lack of vitellogenin synthesis and visibility of interior cells in sugar-fed mosquitoes. In all follicles examined that contained identifiable structures (i.e., nurse cells and oocytes) active caspases were localized to the interior portion of the follicle, especially at the interior interfaces between epithelial and nurse cells as well as the interfaces between nurse cells and oocyte (Figure 8; panel B). In many cases, caspases could be visualized within the remains of the oocyte (Figure 8; panel C). From these observations, it seems that apoptosis (as indicated by active caspase localization) occurs first in the interior cells of the follicle, followed later by the epithelial cells. These observations represent a new detail in the understanding of the process of resorption as previous descriptions were unable to describe events occurring within the follicle possibly because of the obfuscation of interior structures by dense yolk proteins in follicles after a blood-meal (Uchida et al., 2004).

Previous reports have described apoptosis in patches of follicular epithelium cells as preceding resorption of the entire ovarian follicle in Anopheline spp. mosquitoes infected with Plasmodium or artificial immuneelicitors (Ahmed and Hurd, 2006; Hopwood et al., 2001). These observations conflict somewhat with observations made here as well as with previous reports of resorption in Culex pipiens pallens by Uchida et al., (2004). It is possible that the progression of apoptosis and resorption seen in infected Anopheles spp. is a unique feature of Plasmodium spp. infection, is a unique feature of immune response, or is a species-specific response. Also, each of the previous reports 
explored only vitellogenic follicles. It is possible that vitellogenic resorption progresses differently than previtellogenic resorption. More than likely, the discrepancies reported here reflect a more complicated relationship between various cells of the follicle than was previously supposed.

\subsubsection{Conclusions}

By combining abdominal ligations, hormone application and nutritional manipulations we were able to determine that $\mathrm{JH}$ likely mediates reproductive trade-offs in $A$. aegypti during the resting stage by preventing apoptosis and resorption of reproductive tissues in a nutrition-dependant manner. However, some key questions remain: 1) How are the resources from resorbed follicles

being used? 2) Why does sugar feeding that is adequate for lipid synthesis and metabolism not prevent resorption? And finally 3) How does JH prevent follicular resorption during the resting stage?

\subsection{Acknowledgments}

We thank Dr. Marcela Nouzova and Dr. Crisalejandra Rivera for rearing mosquitoes as well as Dr. Jaime Mayoral, Dr. Marcela Nouzova and Mario Perez for critical reading of the manuscript. This work was supported by $\mathrm{NIH}$ grant $\mathrm{Al}$ 45545 to FGN and NIH/NIGMS R25 GM061347 to MEC. 


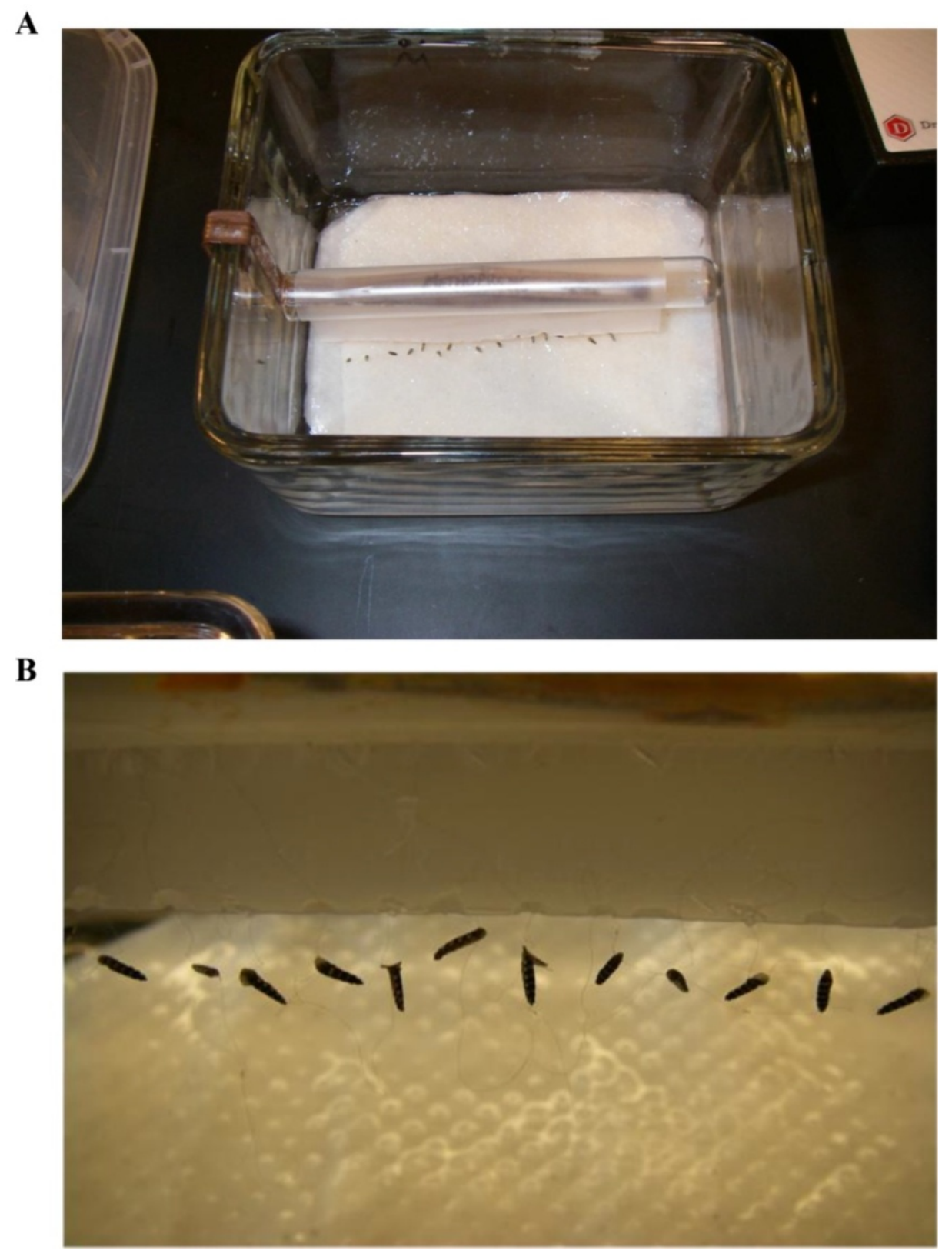

Figure 2: Abdominal ligation preparations. (A) Photograph of the humid glass chamber showing the abdomens hanging from the strip of parafilm. (B) Detail of the abdomen preparations hanging from the strip of parafilm. 

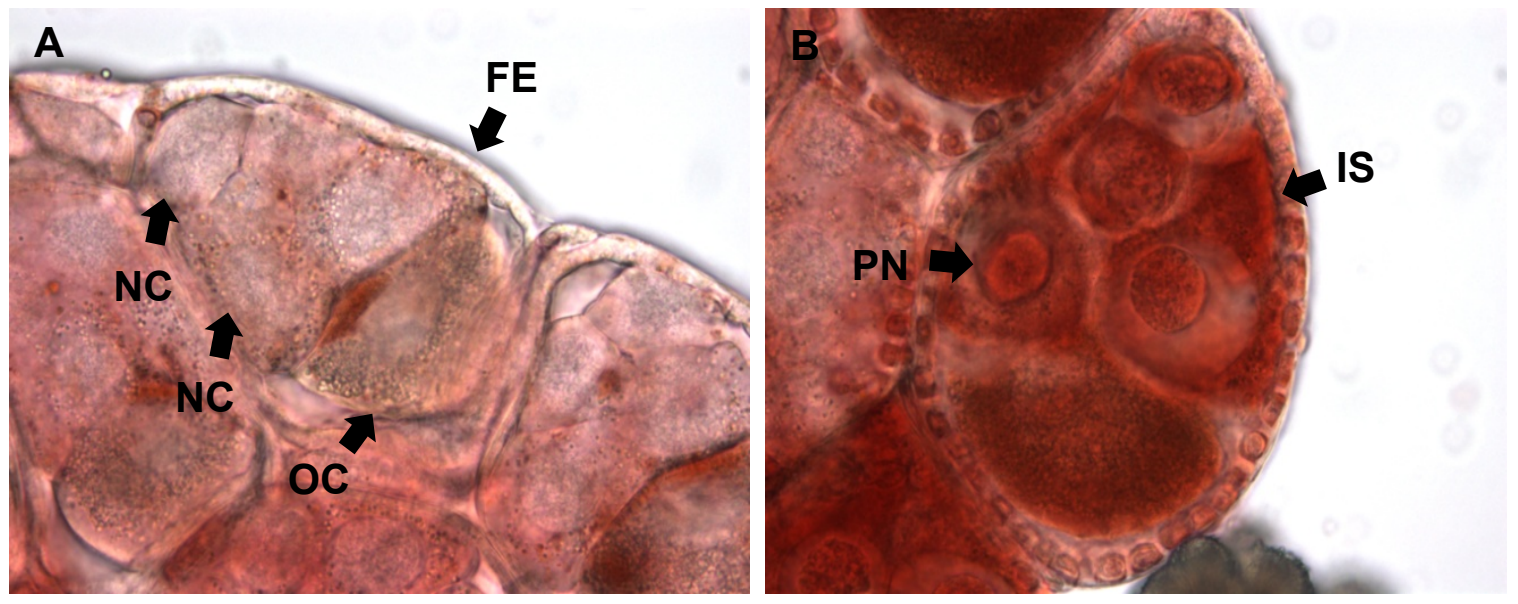

Figure 3: Normal and resorbing follicles stained with neutral red. (A) Image of a normal follicle stained with neutral red: NC: nurse cell, FE: follicular epithelium, OC: oocyte. (B): Image of an apoptotic follicle stained with neutral red: PN: pyknotic nucleus showing condensed chromatin. IS: intercellular space. 
A
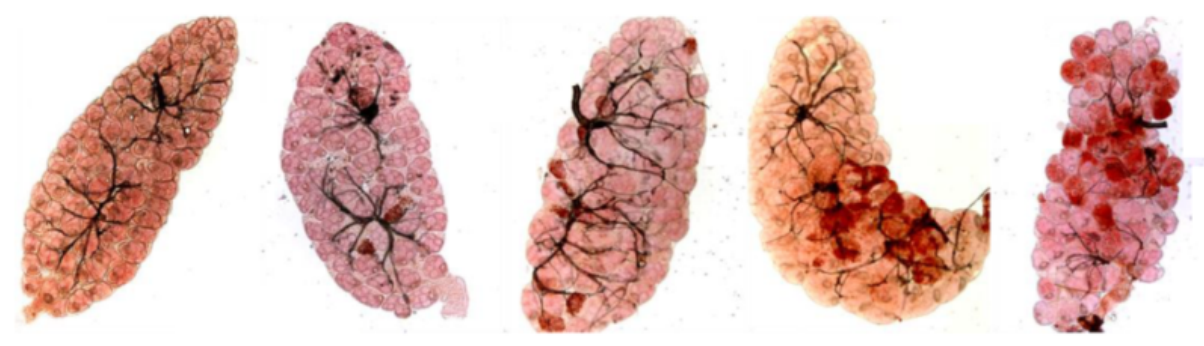

B

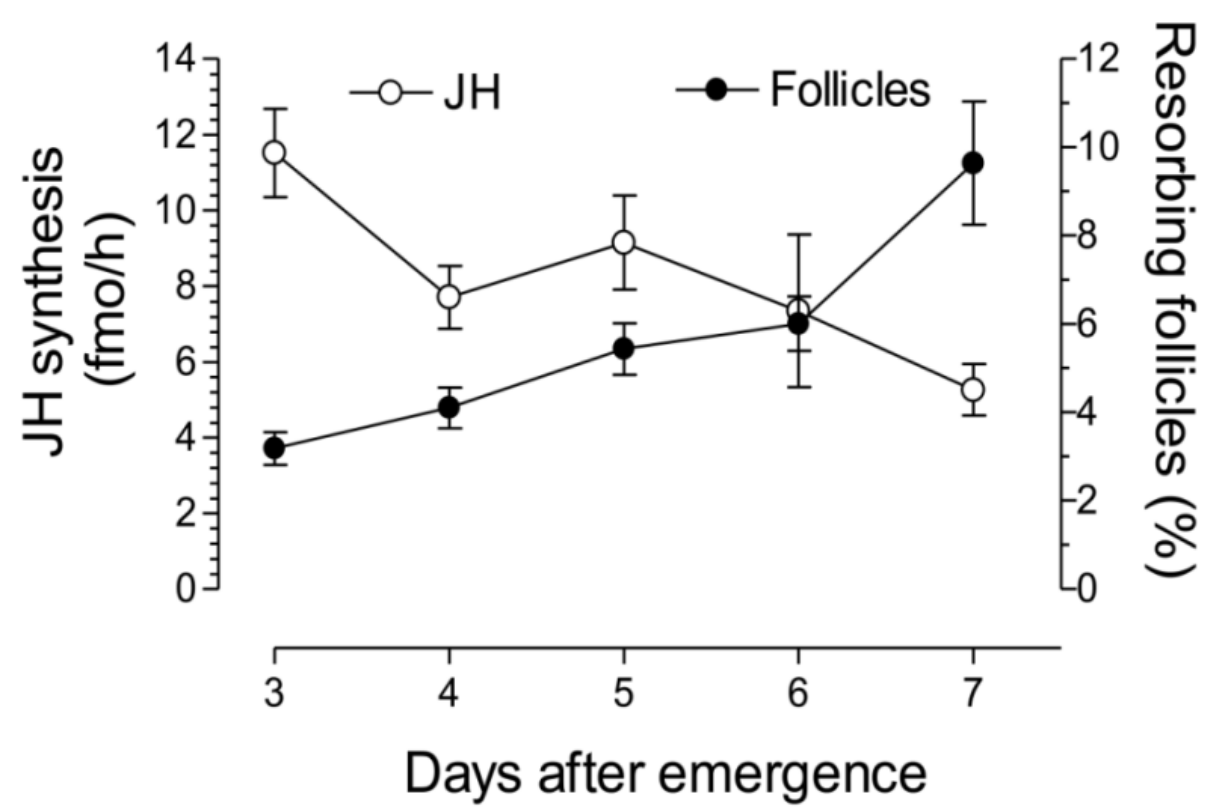

Figure 4: Changes in the rate of previtellogenic follicular resorption. (A) Representative images of the progression of resorption in ovaries from day 3 until day 7 after emergence. Ovaries are stained with neutral red. (B) Average percent of resorbing follicles per day in female mosquitoes fed $3 \%$ sucrose (filled circles) (Mean \pm SEM of five independent replicates of groups of at least 10 ovaries). JH biosynthesis values (empty circles) are from Li et al., 2003, and are expressed as femtomole of JH III per hour. 


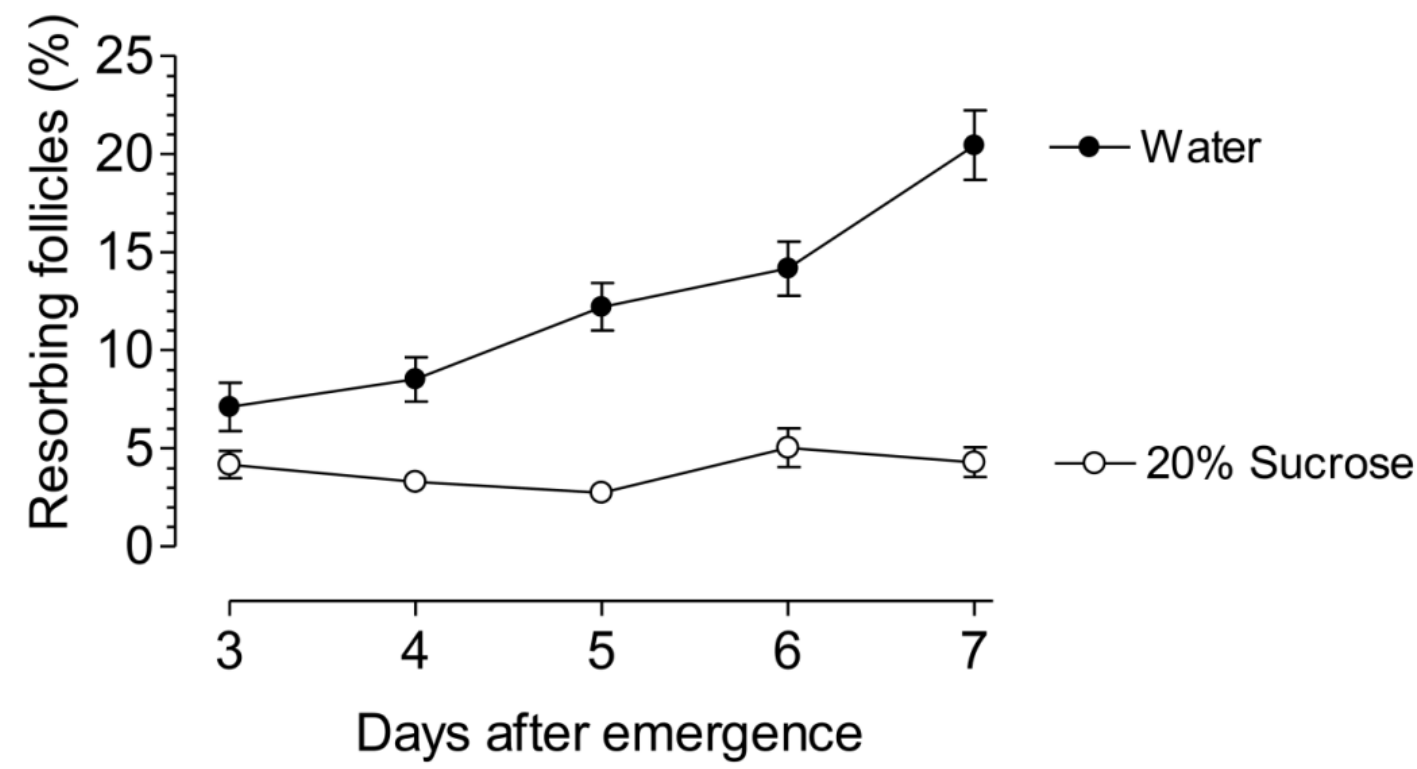

Figure 5: Effect of nutrition on the rate of previtellogenic follicular resorption. Average percent of resorbing follicles from day 3 to day 7 after emergence in females fed with either $20 \%$ sucrose solution (empty circles) or water alone (filled circles). (Mean \pm SEM of three independent replicates of groups of 10 ovaries; unpaired $t$-test; ${ }^{* * *} \mathrm{p} \leq$ 0.001). 


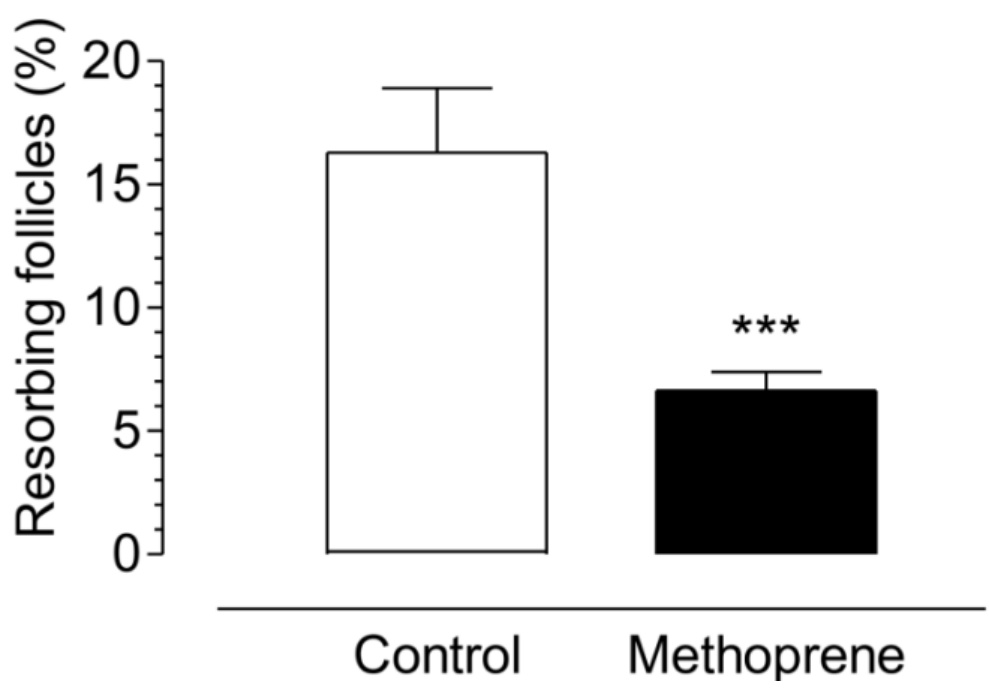

Figure 6: Methoprene prevents follicular resorption in ligated abdomens.. Abdomens were ligated 3 days after emergence and treated with either acetone as a control (empty bar) or $500 \mathrm{ng}$ of methoprene dissolved in acetone (filled bar). Follicular resorption was evaluated after 48 hours. Bars represent the means \pm SEM of four independent replicates of groups of at least 10 ovaries (unpaired $t$-test; ${ }^{* * *} p \leq 0.001$ ). 


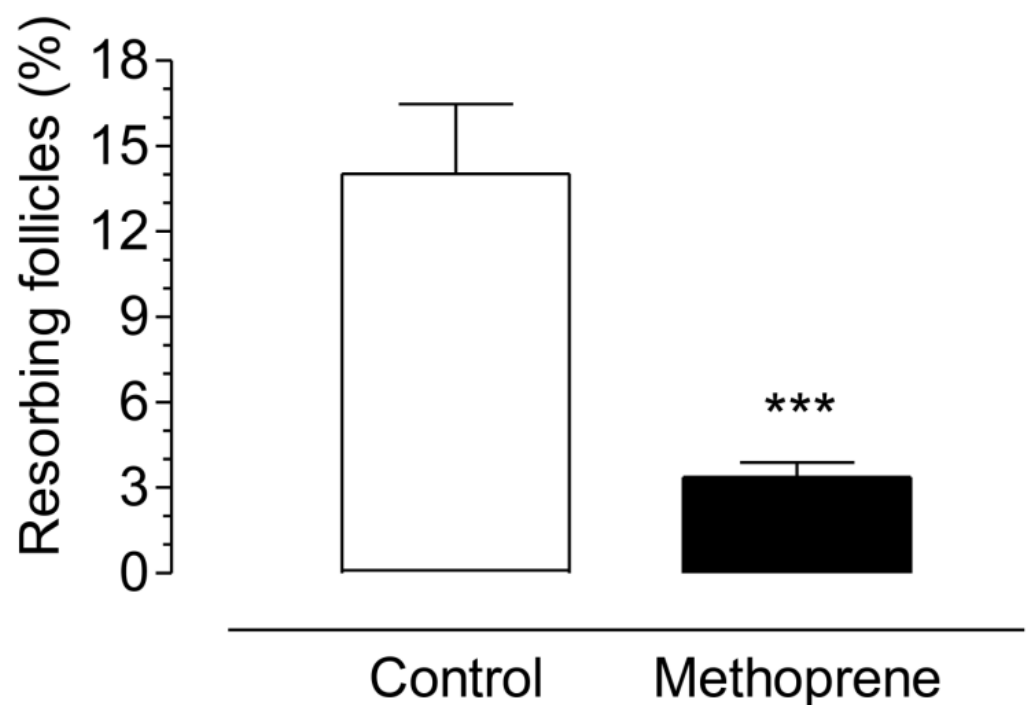

Figure 7: Methoprene prevents follicular resorption in starved mosquitoes. Mosquitoes were fed $3 \%$ sucrose until being treated with either acetone as a control (empty bar) or $500 \mathrm{ng}$ of methoprene dissolved in acetone (filled bar) and then starved. Follicular resorption was evaluated after 48 hours. Bars represent the means \pm SEM of two independent replicates of groups of 30 ovaries (unpaired t-test; ${ }^{* * *} \mathrm{p} \leq$ $0.001)$. 

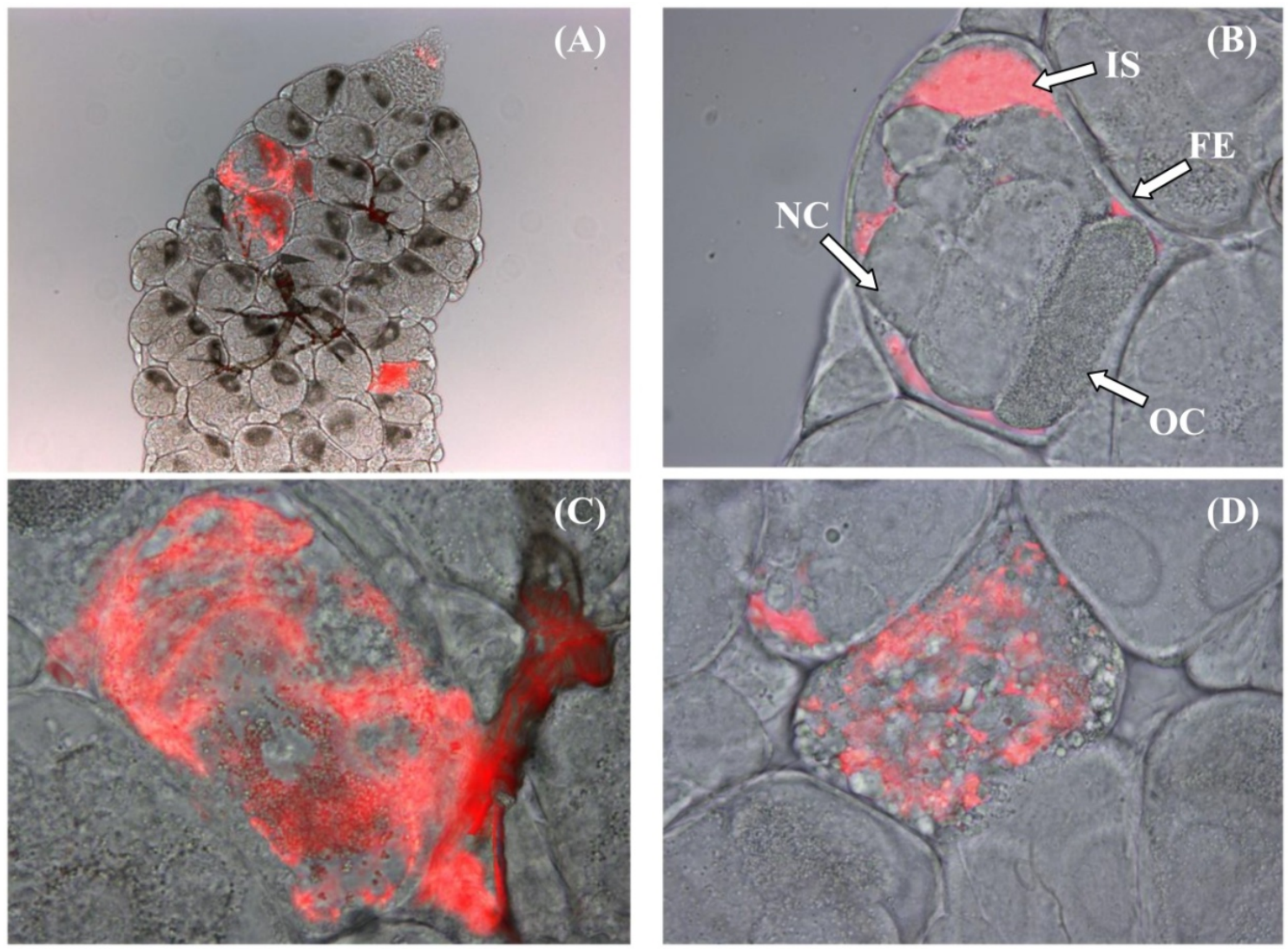

Figure 8: Active caspase localization in previtellogenic follicles. Active caspases were visualized by merging a light micrograph with a fluorescent micrograph. (A) Resorbing follicles can be identified by the presence of pyknotic nuclei and accumulation of a sulforhodamine-labeled caspase inhibitor probe (SR-VAD-FMK). Normally developing follicles fail to accumulate significant amounts of SR-VAD-FMK and show uncondensed nurse cell nuclei. (B) Early resorbing follicles with definable interior structures had active caspases localized solely to intercellular spaces and not within follicle cells, nurse cells or the oocyte. (C) As resorption progresses, interior cell types become degraded and caspases can be visualized throughout the interior of the follicle. (D) In late resorbing follicles all interior structures have been degraded and the remaining cells of the follicular epithelium contain active caspases. FE, Follicular epithelium; NC, Nurse cell; OC, Oocyte; PN, Pyknotic nuclei; IS, Intercellular space. 


\subsection{References}

Ahmed, A.M., Hurd, H. 2006. Immune stimulation and malaria infection impose reproductive costs in Anopheles gambiae via follicular apoptosis. Microbes and Infection. 8, 308-315.

Barrett, E.L.B., Preziosi, R.F., Moore, A.J., Moore, P.J. 2008. Effects of mating delay and nutritional signals on resource recycling in a cyclically breeding cockroach. Journal of Insect Physiology. 54, 25-31.

Bell, W.J., Bohm, M.K. 1975. Oosorption in insects. Biological Reviews. 50, 373396.

Boggs, C.L. 1981. Nutritional and life-history determinants of resource allocation in holometabolous insects. The American Naturalist. 117, 692-709.

Boggs, C.L. 1992. Resource allocation: exploring connections between foraging and life history. Functional Ecology. 6, 508-18.

Boggs, C.L., Ross. C.L. 1993. The effect of adult food limitation on life-history traits in Speyeria mormonia (Lepidoptera, Nymphalidae). Ecology. 74, 433-441.

Borovsky, D., Whisenton, L. R., Thomas, B. R., Fuchs. M.S. 1986. Biosynthesis and distribution of ecdysone and 20-OH-ecdysone in Aedes aegypti. Archives of Insect Biochemistry and Physiology. 3, 19-30.

Briegel, H. 1990. Metabolic relationship between female body size, reserves, and fecundity of Aedes aegypti. Journal of Insect Physiology. 36, 165-172.

Briegel, H., Knüsel, I., Timmermann. S.E. 2001. Aedes aegypti: size, reserves, survival, and flight potential. Journal of Vector Ecology. 26, 21-31.

Briegel, H., Hefti, M., Dimarco, E. 2002. Lipid metabolism during sequential gonotrophic cycles in large and small female Aedes aegypti. Journal of Insect Physiology. 48, 547-554.

Casas, J., Pincebourde, S., Mandon, N., Vannier, F., Poujol, R., Giron, D. 2005. Lifetime nutrient dynamics reveal simultaneous capital and income breeding in a parasitoid. Ecology. 86, 545-554.

Caroci, A., Li, Y., Noriega, F.G. 2004. Reduced juvenile hormone synthesis in mosquitoes with low teneral reserves prevents ovarian previtellogenic development in Aedes aegypti. Journal of Experimental Biology. 207, 2685-2690. 
Carwardine, S.L., Hurd., H. 1997. Effects of Plasmodium Yoelii nigeriensis infection on Anopheles stephensi egg development and resorption. Medical and Veterinary Entomology. 11, 265-269.

Clements, A.N. 1992. The Biology of Mosquitoes, Chapman \& Hall, London.

Clements, A.N., Boocock, M.R., 1984. Ovarian development in mosquitoes: stages of growth and arrest and follicular resorption. Physiological Entomology. 9, 1-8.

Cooper D.M., Thi, E.P., Chamberlain C.M., Pio, F., Lowenberger, C. 2007. Aedes Dronc: a novel ecdysone-inducible caspase in the yellow fever mosquito, Aedes aegypti. Insect Molecular Biology. 16, 563-572.

Feinsod, F.M., Spielman, A. 1980. Nutrient-mediated juvenile hormone secretion in mosquitoes. Journal of Insect Physiology. 26, 113-117.

Flatt T., Tu M.P., Tatar, M. 2005. Hormonal pleiotropy and the juvenile hormone regulation of Drosophila development and life history. BioEssays. 27,9991010.

Foster, W.A. 1995. Mosquito sugar feeding and reproductive energetics. Annual Review of Entomology. 40, 443-474.

Gwadz, R.W., Spielman, A. 1973. Corpus allatum control of ovarian development in Aedes aegypti. Journal of Insect Physiology. 19, 1441-1448.

Hagedorn, H.H., Turner, S., Hagedorn, E.A., Pontecorvo, D., Greenbaun, P., Pfeiffer, D., Wheelock, G. Flanagan, T.R. 1977. Post emergence growth of the ovarian follicles of Aedes aegypti. Journal of Insect Physiology. 23, 203-206.

Hernández-Martínez, S., Mayoral, J.G, Li, Y., Noriega, F.G. $2007 . \quad$ Role of juvenile hormone and allatotropin on nutrient allocation, ovarian development and survivorship in mosquitoes. Journal of Insect Physiology. 53, 230-234.

Hopwood, J.A., Ahmed, A.M., Polwart, A., Williams, G.T., Hurd, H. 2001. Malariainduced apoptosis in mosquito ovaries: a mechanism to control vector egg production. Journal of Experimental Biology. 204, 2773-2780.

Judson, C.L., de Lumen, H.Z. 1976. Some effects of juvenile hormone and analogues on ovarian follicles of the mosquito Aedes aegypti (Diptera: Culicidae). Journal of Medical Entomology. 13, 197-201. 
Lea, A.O. 1963. Some relationships between environment, corpora allata, and egg maturation in Aedine mosquitoes. Journal of Insect Physiology. 9, 793-809.

Lea, A.O., Briegel, H., Lea, H.M. 1978. Arrest, resorption, or maturation of oocytes in Aedes aegypti: dependence on the quantity of blood and the interval between blood meals. Physiological Entomology. 3, 309-316.

Li, Y., Hernandez-Martinez, S., Unnithan, G.C., Feyereisen, R., Noriega. F.G. 2003. Activity of the corpora allata of adult female Aedes aegypti: effects of mating and feeding. Insect Biochemistry and Molecular Biology. 33, 1307-1315.

Moczek, A.P. 1998. Horn polyphenism in the beetle Onthophagus taurus: larval diet quality and plasticity in parental investment determine adult body size and male horn morphology. Behavioral Ecology. 9, 636-641.

Moczek, A.P., Nijhout, H.F. 2004. Trade-offs during the development of primary and secondary sexual traits in a horned beetle. The American Naturalist. 163, 184-191.

Naksathit, A.T., Edman, J.D., Scott, T.W. 1999. Amounts of glycogen, lipid, and sugar in adult female Aedes aegypti (Diptera: Culicidae) fed sucrose. Journal of Medical Entomology. 36, 8-12.

Nayar, J.K., Sauerman, J.D.M. 1971. The effects of diet on life-span, fecundity and flight potential of Aedes taeniorhynchus adults. Journal of Medical Entomology. 8, 506-513.

Nayar, J.K., van Handel, E., 1971. The fuel for sustained mosquito flight. Journal of Insect Physiology. 17, 471-481.

Nijhout, H.F., Wheeler. D.E. 1982. Juvenile hormone and the physiological basis for insect polymorphisms. The Quarterly Review of Biology. 57, 109-133.

Noriega, F.G. Shah, D.K. Wells, M.A., 1997. Juvenile hormone controls early trypsin gene transcription in the midgut of Aedes aegypti. Insect Molecular Biology. 6, 63-66.

Noriega, F.G., 2004. Nutritional regulation of JH synthesis: a mechanism to control reproductive maturation in mosquitoes? Insect Biochemistry and Molecular Biology. 34, 687-693.

Ohgushi, T., 1996. A reproductive tradeoff in an herbivorous lady beetle: egg resorption and female survival. Oecologia.106, 345-351. 
Oliveira, G.A., Baptista, D.L., Guimaraes-Motta, H., Almeida, I.C., Masuda, H., Atella, G.C. 2006. Flight-oogenesis syndrome in a blood sucking bug: biochemical aspects of lipid metabolism. Archives of Insect Biochemistry and Physiology. 62,164-175.

Osawa, N., 2005. The effect of prey availability on ovarian development and oosorption in the ladybird beetle Harmonia axyridis (Coleoptera: Coccinellidae). European Journal of Entomology. 102, 503-511.

Parker, E.R., Pianka, W.S., 1975. Age specific reproductive tactics. The American Naturalist. 109, 453-464.

Raikhel, A.S., Lea, A.O., 1984. Hormone-mediated formation of the endocytic complex in mosquito oocytes. General and Comparative Endocrinology. $57,422-433$.

Raikhel, A.S., Lea, A.O., 1991. Control of follicular epithelium development and vitelline envelope formation in the mosquito: role of juvenile hormone and 20-hydroxyecdysone. Tissue \& Cell. 23, 577-591.

Rantala, M.J., Vainikka, A., Kortet, R., 2003. The role of juvenile hormone in immune function and pheromone production trade-offs: a test of the immuno-competence handicap principle. Proceedings of the Royal Society B. $270,2257-2261$.

Richard, R., Casas., J. 2009. Stochasticity and controllability of nutrient sources in foraging: host-feeding and egg resorption in parasitoids. Ecological Monographs. 79, 465-483.

Rivero, A., Giron, D., Casas, J. 2001. Lifetime allocation of juvenile and adult nutritional resources to egg production in a Holometabolous insect. Proceedings of National Academy of Sciences. 268, 1231-1237.

Rosenheim, J.A., Heimpel, G.E., Mangel, M. 2000. Egg maturation, egg resorption and the costliness of transient egg limitation in insects. Proceedings of the Royal Society B. 267, 1565-1573.

Schal, C., Chiang, A.-S., Burns, E.L., Gado, M., Cooper, R.A. 1993. Role of the brain in juvenile hormone synthesis and oocyte development: effects of dietary protein in the cockroach Blattella germanica (L.). Journal of Insect Physiology. 39, 303-313.

Shapiro, A.B., Wheelock, G.D., Hagedorn, H.H., Baker, F.C, Tsai, L.W., Schooley, D.A. 1986. JH and juvenile esterase in adult females of the mosquito A. aegypti. Journal of Insect Physiology. 32, 867-877. 
Söller, M., Bownes, M., Kubli. M.E., 1999. Control of oocyte maturation in sexually mature Drosophila females. Developmental Biology. 208, 337351.

Spindler, K.D., Honl, C., Tremmel, C.H. Braun, S., Ruff, H., Spindler-Barth, M. 2009. Ecdysteroid hormone action. Cellular and Molecular Life Sciences. $66,3837-3850$.

Stevens, D.J., Hansell, M.H., Monaghan, P. 2000. Developmental trade-offs and life histories: strategic allocation of resources in caddis flies. Proceedings of the Royal Society B, 267, 1511-1515.

Telang, A., Li, Y., Noriega, F.G., Brown, M.R. 2006. Effects of larval nutrition on the endocrinology of mosquito egg development. Journal of Experimental Biology. 209, 645-655.

Terashima, J., Bownes, M. 2004. Translating available food into the number of eggs laid by Drosophila melanogaster. Genetics. 167, 1711-1719.

Terashima, J., Takaki, K., Sakurai, S., Bownes, M. 2005. Nutritional status affects 20-hydroxyecdysone concentration and progression of oogenesis in Drosophila melanogaster. Journal of Endocrinology. 187, 69-79.

Tobe, S.S., Chapman, C.S. 1979. The effects of starvation and subsequent feeding on juvenile hormone synthesis and oocyte growth in Schistocerca americana gregaria. Journal of Insect Physiology. 25, 701-708.

Troy, S., Anderson, W.A., Spielman, A. 1975. Lipid content of maturing ovaries of Aedes aegypti mosquitoes. Comparative Biochemistry and Physiology B. $50,457-461$.

Trumbo, S.T., Robinson, G.E. 2004. Nutrition, hormones and life history in burying beetles. Journal of Insect Physiology. 50, 383-391.

Uchida, K., Nishizuka, M., Ohmori, D., Ueno, T., Eshita, Y., Fukunaga, A. 2004. Follicular epithelial cell apoptosis of atretic follicles within developing ovaries of the mosquito Culex pipiens pallens. Journal of Insect Physiology. 50, 903-912.

Wheeler, D.E. 1996. The role of nourishment in oogenesis. Annual Review of Entomology. 41, 345-379.

Winckler, J. 1974. Vital staining of lysosomes and other cell organelles of the rat with neutral red. Progress in histochemistry and cytochemistry. 6, 1-91. 
Wu, Y., Parthasarathy, R., Bai, H. Palli., S.R. 2006. Mechanisms of midgut remodeling: juvenile hormone analog methoprene blocks midgut metamorphosis by modulating ecdysone action. Mechanisms of Development. 123, 530-547.

Yin, C.M., Qin, W.H., Stoffolano Jr., J.G. 1999. Regulation of mating behaviour by nutrition and the corpus allatum in both male and female Phormia regina (Meigen). Journal of Insect Physiology. 45, 815-822.

Ziegler, R., 1997. Lipid synthesis by ovaries and fat body of Aedes aegypti (Diptera: Culicidae). European Journal of Entomology. 94, 385-391.

Ziegler, R., Van Antwerpen., R. 2006. Lipid uptake by insect oocytes. Insect Biochemistry and Molecular Biology. 36, 264-272.

Ziegler, R., Ibrahim, M.M. 2001. Formation of lipid reserves in fat body and eggs of the yellow fever mosquito, Aedes aegypti. Journal of Insect Physiology. $47,623-627$.

Zhou, G., Flowers, M., Friedrich, K., Horton, J., Pennington, J., Wells, M.A. 2004. Metabolic fate of [14C]-labeled meal protein amino acids in Aedes aegypti mosquitoes. Journal of Insect Physiology. 50, 337-349. 


\section{Chapter 4: The fate of follicles after a blood meal is dependent on previtellogenic nutrition and juvenile hormone in Aedes aegypti.}

\subsection{Abstract}

Juvenile hormone $(\mathrm{JH})$ mediates the relationship between fecundity and nutrition during the gonotrophic cycle of the mosquito in three ways: 1) by regulating initial previtellogenic development 2) by mediating previtellogenic resorption of follicles 3) and by altering intrinsic previtellogenic follicle "quality", physiology, and competitiveness thereby predetermining the fate of follicles after a blood meal. To support a role for $\mathrm{JH}$ in mediating the response of ovarian follicles after a blood meal, we explored three main questions: (1) Do changes in nutrition during the previtellogenic resting stage lead to relevant biochemical and molecular changes in the previtellogenic ovary? (2) Do hormonal manipulations during the previtellogenic resting stage lead to the same biochemical and molecular changes? (3) Does nutrition and hormones during the previtellogenic resting stage affect vitellogenic resorption and reproductive output? We examined the accumulation of neutral lipids in the previtellogenic ovary as well as the previtellogenic expression of genes integral to endocytosis and oocyte development such as the: vitellogenin receptor $(A a \vee g R)$, lipophorin receptor (AaLpRov), heavy-chain clathrin $(\mathrm{AaCHC})$, and ribosomal protein L32 (rpL32) under various previtellogenic nutritional and hormonal conditions. The abundance of mRNA's as well as neutral lipid content increased within the previtellogenic ovary as previtellogenic mosquitoes were offered increasing sucrose concentrations. Methoprene application mimicked the effect of offering 
the highest sucrose concentrations on mRNA abundance and lipid accumulation in the previtellogenic ovary. These same nutritional and hormonal manipulations altered the extent of vitellogenic resorption. Mosquitoes offered $20 \%$ sucrose during the previtellogenic resting stage had nearly 3 times less vitellogenic resorption than mosquitoes offered $3 \%$ sucrose despite taking smaller blood meals and developed $\sim 10 \%$ more eggs during the first gonotrophic cycle. Mosquitoes treated with $\mathrm{JH}$ III during the previtellogenic resting stage and then offered a blood meal had a $\sim 40 \%$ reduction in the amount of vitellogenic resorption and developed $\sim 12 \%$ more eggs than control females. Taken together, these results suggest that previtellogenic nutrition alters the extent and pattern of resorption after a blood meal through the effect of $\mathrm{JH}$ on mRNA abundance and lipid accumulation in previtellogenic follicles.

\subsection{Introduction}

A broad examination of the various life history strategies among insects repeatedly demonstrates that reproductive output and nutritional status are coordinated to ensure survival and maximize long-term fitness. When nutrition is limited or environmental conditions are not ideal, reproductive trade-offs frequently manifest through the resorption of reproductive tissue (also described as: oosorption, follicular resorption or follicular atresia) (Reviewed in Bell and Bohm, 1975). Previous work has indicated that juvenile hormone $(\mathrm{JH})$ is a key endocrine participant in the mediation of follicular resorption in response to nutritional limitation (Highnam et al., 1963a; Bell, 1971; Bell and Bohm, 1975; Tobe and Chapman, 1979; Weaver and Pratt, 1981; Clifton and Noriega, 2011). 
In insects that produce eggs continuously and sequentially, the relationship between $\mathrm{JH}$, follicular resorption, and nutrition is usually direct and causal. Yolk protein production and oogenesis are often dependent on $\mathrm{JH}$ synthesis and overall nutritional status. When nutrition is limited, JH synthesis ceases, yolk protein synthesis is reduced and ovarian oosorption increases (Bell, 1971; Bell and Bohm, 1975; Tobe and Chapman, 1979; Weaver and Pratt, 1981). By coordinating the relationship between nutritional status and reproductive output through follicular resorption, $\mathrm{JH}$ participates directly in the reproductive decisions that inform an insect's life-history strategy (reviewed in Flatt, 2005; Zera and Zhao, 2004; Harshman and Zera, 2007; Fronstin and Hatle, 2008). However, a conceptual framework that depends on a close linkage between $\mathrm{JH}$, nutrition, and resorption cannot fully explain how fecundity and nutrition are coordinated in mosquitoes such as Aedes aegypti where oogenesis is discontinuous, dependent on a blood meal, and controlled by alternating titers of $\mathrm{JH}$ and ecdysone.

In anautogenic mosquitoes, reductions in fecundity through follicular resorption are known to occur during two main developmental stages: (1) a sugar-feeding previtellogenic stage governed primarily by $\mathrm{JH}$ (Gwadz and Spielman, 1973; Feinsod and Spielman 1980; Raikhel and Lea 1985; reviewed in Klowden, 1997) (2) and a blood-meal dependent vitellogenic stage governed primarily by ecdysteroids (Spielman et al., 1971; Fallon et al., 1974; Clements, 1992; reviewed in Klowden, 1997). After initial ovarian maturation is complete (60 hours post eclosion), the mosquito enters a previtellogenic resting stage that lasts until a blood meal is found (Clements, 1992). During this period, JH 
synthetic rates slowly decline from their peak while ovarian follicles are resorbed through an apoptosis-like mechanism (Li et al., 2003; Clifton and Noriega, 2011). Resorption during this time is dependent on the quality of nutrition obtained through sugar feeding as well as $\mathrm{JH}$. Sugar feeding and nutrition have important implications for fecundity during the previtellogenic resting stage as up to $20 \%$ of follicles can be resorbed in starved mosquitoes (Clifton and Noriega, 2011).

The first day after a blood meal in mosquitoes is marked by a rapid decrease in $\mathrm{JH}$ synthesis to less than $5 \mathrm{fmol} /$ pair CA/hour, the lowest level seen during a gonotrophic cycle in $A$. aegypti (Li et al., 2003). By 20 hours after a blood meal, ecdysteroid titers in the whole body of the mosquito have increased at least 5-fold from their original levels (Greenplate et al., 1985). In response to amino acids from the blood meal (Hansen et al., 2004) and high ecdysteroid titers (Martin et al., 2001) the fat body synthesizes vitellogenin and other yolk proteins (Raikhel and Lea, 1983; Cho et al., 1991, 1999; Sun et al., 2000) and mobilizes between $\sim 50-80 \%$ of previtellogenic fat body lipid reserves for energy production and for incorporation into the oocytes (Briegel et al., 2002; Zeigler and Ibrahim, 2000; Zhou et al., 2004a). Significant nutritional partitioning of resources occurs after a blood meal as previtellogenic nutrition is not utilized in the same manner and cannot be considered interchangeable with blood meal derived nutrition during oogenesis (Zhou et al., 2004a; 2004b). In Aedes aegypti, 19\% of radiolabeled amino acids within the blood meal are deaminated and returned to the fat body to replace lipids transferred to the oocytes, while only $10 \%$ of the amino acids in a blood meal are directly incorporated into eggs (Zhou et al., 
2004a; 2004b). The substantial remaining portion of the blood meal is used in energy production (43\%) or is excreted as waste (29\%) (Zhou et al., 2004b). Since the nutritional content of eggs is substantially derived from previtellogenic nutritional reserves, it is not clear how reproductive output during vitellogenesis can be coordinated (via vitellogenic resorption) with these previtellogenic nutritional reserves in the absence of any significant $\mathrm{JH}$ synthesis.

After a blood meal, the oocytes and nurse cells show rapid increases in the synthesis of components required for the endocytosis of yolk proteins and lipids such as the: lipophorin receptor (Cheon et al., 2001; Seo et al., 2003; Sun et al., 2000), vitellogenin receptor (Sappington et al., 1996; Cho and Raikhel, 2001), and heavy-chain clathrin (Kokoza and Raikhel, 1997a; Kokoza et al., 1997). The synthesis of endocytotic components in the follicles occurs in parallel with the synthesis and transfer of yolk components by the fat body (Roth and Porter, 1964; Koller and Raikhel, 1991; reviewed in Raikhel and Dhadialla, 1992). Nearly all follicles that are remaining at the time of a blood meal will begin incorporating yolk proteins and lipids (Clements and Boocock, 1984). However, up to $27 \%$ of follicles will later become resorbed despite sometimes advanced vitellogenic development (Lea et al., 1978; Clements and Boocock, 1984). The mechanism or factors that allows one follicle to develop to maturity while another resorbs in the same ovary has not been described but has been suggested to involve "competition" between developing follicles for limited yolk components with the disadvantaged follicles eventually being resorbed (Highnam et al., 1963b; Schlaeger et al., 1974; Bell and Bohm, 1975) and their yolk components 
possibly returned to the pool of available nutrients (Bell, 1971). By 72 hours post blood meal, a synchronous batch of eggs, adjusted over two developmental stages according to nutritional status, is ready for oviposition.

It is well documented that the final quantity of eggs produced (and as a corollary, the amount of vitellogenic resorption) is dependent in part on the quantity of blood ingested when comparing mosquitoes with equal previtellogenic nutritional reserves (Woke et al., 1956, Colless and Chellapah 1960, Jalil 1974; Lea et al., 1978). Other work has shown that mosquitoes with low previtellogenic reserves will take substantially larger blood meals than high reserve mosquitoes but will still fail to develop an equal number of eggs. Conversely, mosquitoes with high reserves will take smaller blood meals but will always develop more eggs than low reserve mosquitoes (Nayar and Sauerman, 1975; Mostowy and Foster 2004). Although a large blood meal can affect fecundity, it cannot prevent all vitellogenic resorption (Lea et al., 1978; Clements and Boocock, 1984) nor can it rescue the fecundity of mosquitoes with low nutritional reserves (Mostowy and Foster, 2004). These results, when taken together, suggest that most decisions about final reproductive output are made in response to nutrition during the previtellogenic resting stage and this information is integrated with blood feeding behavior to determine final fecundity as previously suggested in a conceptual model by Mostowy and Foster (2004). However, no hormonal or molecular mechanism has been elucidated which can explain how nutrition during the previtellogenic resting stage can affect blood feeding behavior or vitellogenic resorption. 
Previous work in our laboratory has demonstrated that $\mathrm{JH}$ likely mediates follicular resorption in response to nutrition during the previtellogenic resting stage (Clifton and Noriega, 2011). During the course of that work it became apparent that nutritional and hormonal manipulations resulted in clear morphological changes to the previtellogenic ovary beyond only follicular resorption. These observations suggested that $\mathrm{JH}$ may mediate the relationship between fecundity and nutrition during the gonotrophic cycle of the mosquito in two ways: 1) through immediate previtellogenic resorption of follicles as previous work has shown (Clifton and Noriega, 2011), 2) and by altering intrinsic previtellogenic follicle "quality", physiology, and competitiveness during vitellogenesis thereby predetermining the fate of follicles after a blood meal. A hypothetical relationship between follicle fate and $\mathrm{JH}$ provides an endocrine mechanism whereby $\mathrm{JH}$ can mediate the full course of reproductive trade-offs in anautogenic mosquitoes during the previtellogenic and vitellogenic stages despite being nearly completely absent when many of these reproductive tradeoffs are actually made (i.e., during vitellogenesis) (Li et al., 2003). To support this hypothesis, we explored three main questions: (1) Do changes in nutrition during the previtellogenic resting stage lead to relevant biochemical and molecular changes in the previtellogenic ovary? (2) Do hormonal manipulations during the previtellogenic resting stage lead to the same biochemical and molecular changes? (3) Does nutrition and hormones during the resting stage affect vitellogenic resorption and reproductive output? Together these questions demonstrate how the nutritional and hormonal conditions before a blood meal 
affect events after a blood meal and begin to explain one endocrine mechanism whereby previtellogenic nutrition (sugar feeding) is integrated with blood meal derived nutrition to determine fecundity.

\subsection{Methods}

\subsubsection{Nutritional manipulations of insects}

A colony of $A$. aegypti of the Rockefeller strain was maintained at $28^{\circ} \mathrm{C}$ with $80 \%$ relative humidity under a $16 \mathrm{~h}$ day $-8 \mathrm{~h}$ night regime. To control for any possible mating effects and ensure complete mating, males and females

emerging during the same 15 hour overnight period were collected and transferred to enclosures containing a $\sim 2: 1$ excess of male mosquitoes. All mosquitoes were offered a cotton pad soaked in $3 \%$ sucrose solution for 2 days. On the third day post-eclosion, all mosquitoes were switched to one of three conditions: water only, $3 \%$ sucrose or $20 \%$ sucrose solution before being assayed.

\subsubsection{Hormonal manipulations of insects}

At 3 days post-emergence, randomly selected mosquitoes reared as previously described were cold anesthetized and topically treated with either 500 ng of methoprene (Zeocon Co., Palo Alto, CA) dissolved in $0.5 \mu \mathrm{L}$ acetone or with $0.5 \mu \mathrm{L}$ acetone alone. Mosquitoes were returned to a cotton pad soaked in $3 \%$ sucrose solution for 2 days until being dissected for qPCR or lipid quantification. Mosquitoes treated with JH III (Sigma-Aldrich, St. Louis, MO) were cold anesthetized at 3 days old and topically treated with $316 \mathrm{ng}$ of $\mathrm{JH}$ III dissolved in $1 \mu \mathrm{L}$ acetone or with $1 \mu \mathrm{L}$ acetone alone. At 4 days old mosquitoes 
were treated a second time with $\mathrm{JH}$ III or acetone. Mosquitoes treated with $\mathrm{JH}$ III or acetone were blood fed on day 5 and the extent of resorption during oogenesis as well as final reproductive output was determined.

\subsubsection{Ovarian triglyceride assay}

Lipids were detected in the ovaries of mosquitoes maintained under the described experimental conditions using a triglyceride quantification kit (K622100, Biovision, Mountain View, CA). In this kit, mono-, di- and triglycerides are hydrolyzed into glycerol and free fatty acids. Glycerol is then oxidized to generate a product which reacts with a colormetric probe. The absorbance of the colormetric probe can then be read at OD 570nm. For each experimental treatment, 5 pairs of ovaries were dissected in Aedes physiological saline (APS) $\left(\mathrm{MgCl}_{2} 0.6 \mathrm{mM} ; \mathrm{KCl} 4.0 \mathrm{mM} \mathrm{NaHCO}{ }_{3} 1.8 \mathrm{mM} ; \mathrm{NaCl} 150.0\right.$ mM; HEPES 25.0 $\mathrm{mM} ; \mathrm{CaCl}_{2} 1.7 \mathrm{mM}$ ). The ovaries were thoroughly cleaned of any contaminating fat body tissue, rinsed with PBS and placed in $100 \mu \mathrm{L}$ of $5 \%$ nonidet P-40 detergent solution. After brief sonication, any remaining ovary lysate on the probe of the sonicator was rinsed back into the sample with $400 \mu \mathrm{L}$ of $d \mathrm{dd}_{2} \mathrm{O}$. The ovarian lysate was then processed according to the kit instructions. Absorbance was read in a cuvette with a Nanodrop 2000c spectrophotometer (Thermo Fisher Scientific, Waltham, MA). The lipase-negative glycerol control was used to blank the Nanodrop thereby subtracting background glycerol.

\subsubsection{Ovarian micrographs and staining with Oil red-O}

Ovaries from mosquitoes were dissected in APS before being fixed in $4 \%$ paraformaldehyde for 1 hour. The formaldehyde was replaced with $100 \mu \mathrm{L}$ of Oil 
red-O working solution for 1 hour. Oil red-O specifically stains triglycerides and cholesteryl oleate and no other lipids (Ramirez-Zacarias et al., 1992). The ovaries were removed from the stain and rinsed thoroughly in PBS before being placed under a coverslip and photographed. Photographs were taken of the previtellogenic ovaries using a DM 5500 B Leica fluorescence microscope, a Leica DFC 310 FX mounted camera and Leica LAS imaging software.

\subsubsection{Quantitative Realtime qPCR}

Total RNA was isolated from ovaries using RNA-binding glass powder as previously described (Noriega and Wells, 1993). Contaminating genomic DNA was removed using the DNA-free ${ }^{\mathrm{TM}}$ kit (Ambion, Austin, TX). Reverse transcription was carried out using the Verso cDNA Kit (Thermo Fisher Scientific Inc., Waltham, MA) by an oligo dT priming method according to the manufacturer's recommendations, using $300 \mathrm{ng}$ of total RNA in $20 \mu \mathrm{l}$ reactions. Real time qPCR was performed with the 7300 Real Time PCR System using $\operatorname{TaqMan}^{\circledR}$ Gene Expression Assays together with TaqMan ${ }^{\circledR}$ Universal PCR Master Mix (Applied Biosystems, Foster City, CA). The primer probes sequences for the 60S ribosomal protein L32 (rpL32, AAEL003396, VectorBase) Ovaryspecific Lipophorin receptor (AaLpRov, AF355595, VectorBase) Heavy-chain clathrin (AaCHC, AAEL013614-RA, VectorBase) and the vitellogenin receptor (AaVgR, AAEL007657, VectorBase) genes are provided in table 1.

Primer/probes were synthesized by Applied Biosystems and reactions were done in a $20 \mu \mathrm{l}$ volume according to manufacturer recommendations for Custom TaqMan ${ }^{\circledR}$ Gene Expression Assays. Reactions were run in triplicate 
using $1 \mu \mathrm{l}$ of cDNA per reaction. Standard curves to quantify relative gene copy number were made from serial dilutions of plasmids containing rpL32, AaVgR, AaLpRov, and AaCHC $(300,000,30,000,3000,300$ and 30 copies of a plasmid per reaction). Real time data were collected by 7300 System SDS Software and analyzed in Microsoft Excel. Because many aspects of ovary and follicle physiology are heavily dependent on nutrition and hormones, traditional methods of normalization (i.e. normalizing to ribosomal, actin, or other housekeeping genes) biases the expression changes observed in previtellogenic oocytes (reviewed in Wrenzycki and Niemann, 2007). Therefore, an alternative approach to normalizing mRNA abundance is required when working with oocytes (Wrenzycki and Niemann, 2007). All genes were normalized to the average total follicle count of mosquitoes collected in triplicate of the same age and treatment to represent gene copy per average follicle.

\subsubsection{Determination of blood meal mass}

Mosquitoes from a common cohort were reared under the nutritional conditions previously described until 5 days old and divided into two groups. Female mosquitoes in the blood fed group were starved for 4-5 hours before being offered porcine blood equilibrated to $37{ }^{\circ} \mathrm{C}$ with ATP added to a final concentration of $1 \mathrm{mM}$ immediately before use. After feeding, mosquitoes from both the fed and unfed group were dried in an incubator at $65^{\circ} \mathrm{C}$ for 5 days before measurement. The dry mass of the blood meal was calculated as the average dry mass of mosquitoes before a blood meal subtracted from the 
average dry mass of mosquitoes after a blood meal. At least 20 mosquitoes per treatment were dried and weighed.

\subsubsection{Observations of ovarian resorption and final egg output}

Mosquitoes within each feeding and hormonal regime were blood fed as previously described. Mosquitoes from at least two independent replicates were anesthetized by chilling for $5-10 \mathrm{~min}$ at $4^{\circ} \mathrm{C}$ prior to dissection. At 24 hours after a blood meal, ovaries were dissected, rinsed in APS and stained with $0.5 \%$ neutral red solution in acetate buffer at pH 5.2 (Sigma-Aldrich, St. Louis, MO) for 10 s to visualize resorbing follicles. Neutral red stains the lysosomes associated with resorbing follicles and can clearly indicate follicle status (Winckler, 1974; Bell and Bohm, 1975; Clements and Boocock, 1984; Hopwood et al., 2001). The ovaries were rinsed a second time in APS and placed under a coverslip. Photographs were taken as described in section 2.4. Ovaries were later scored using Leica LAS imaging software for total follicle count and also for the presence of any resorbing follicles. To determine total egg output, mosquitoes fed $20 \%$ sucrose and $3 \%$ sucrose as well as mosquitoes treated with acetone and $\mathrm{JH}$ III were blood fed in triplicate as previously described and allowed to lay eggs on paper. The total number of eggs was counted by dissecting microscope and divided by the number of females observed to have blood fed to determine average number of eggs per female. 12 days after oviposition, eggs from all treatments were hatched and the total number of unhatched eggs was counted to determine egg viability. 


\subsection{Results}

\subsubsection{Ovary morphology reflects overall nutritional reserves}

The effect of nutrition on previtellogenic ovaries was explored my maintaining mosquitoes under three nutritional regimes: (1) water alone, (2) 3\% sucrose in water, and (3) $20 \%$ sucrose in water. Ovaries from mosquitoes reared under such conditions show differences in follicle size, oocyte size, oocyte composition (as indicated by oil red-O staining of neutral lipids), and overall morphology (Figure 9A). Ovarian morphology appears to be reflective of the overall nutritional reserves of mosquitoes as indicated by the dry mass of mosquitoes kept under varying nutritional regimes (Figure 9B). Dry mass can be a suitable proxy for indicating nutritional reserves (Mostowy and Foster, 2004). Mosquitoes kept under increasing sucrose concentrations show statistically significant increases in dry body mass for each concentration offered (unpaired ttest; $\mathrm{P}<.001)$. Mosquitoes offered $20 \%$ sucrose accumulated nearly double the dry mass of those mosquitoes offered water alone (Figure 9B).

\subsubsection{Previtellogenic nutrition alters neutral lipid content of ovaries}

To investigate the apparent differences in ovarian and follicle morphology more thoroughly, a neutral lipid specific stain (Oil red-O) was used to stain the ovaries and visualize the accumulation of lipids in the oocytes. Ovaries from mosquitoes fed $20 \%$ sucrose concentrations show increased lipid accumulation as compared to those fed water or $3 \%$ sucrose (Figure $10 \mathrm{~A}$ ). The densest accumulation of neutral lipids appears to be in the oocyte followed by the proximal nurse cells (Figure 10A). The nurse cells furthest from the oocyte show 
less association with accumulated lipids. Accumulations of lipids within the oocyte appear to be most pronounced in mosquitoes fed the highest sucrose concentrations (Figure 10A).

Since the content of lipids in the ovary appeared to change depending on previtellogenic nutrition, ovarian lipids may be an important energy source for transcriptional, translational and endocytotic activities during vitellogenesis. To begin exploring this possibility, the neutral lipid content of ovaries was quantified through the modification of a blood serum triglyceride quantification kit. Ovaries from mosquitoes fed $20 \%$ sucrose contained the highest content of stored neutral lipids at 581 pmol./ female (Figure 10B). This two-fold increase in ovarian lipids is significantly higher than mosquitoes fed either $3 \%$ sucrose or water alone (Figure 10B; $P<.0001$; Mann-Whitney test). Feeding $3 \%$ sucrose did not significantly increase stored ovarian lipids over those fed water alone (Figure 10B).

\subsubsection{Previtellogenic nutrition alters expression of genes important for vitellogenesis}

In conjunction with an examination of the energetic capacity of the follicles (i.e. stored lipids) we hypothesized that the expression of other cellular components important during vitellogenesis would be dependent on previtellogenic nutritional status. We chose to examine the expression of a ribosomal protein (60s subunit, rpL32) required for translation and three additional components required for the massive endocytosis associated with vitellogenesis but also expressed in lower levels during the previtellogenic resting stage: (1) The vitellogenin receptor $(A a \vee g R),(2)$ the ovary-specific isoform of the 
lipophorin receptor (AaLpRov), and (3) an ovary-specific form of Heavy-Chain Clathrin $(\mathrm{AaCHC})$. For almost every gene and treatment, the number of transcripts per follicle increased as sucrose concentration increased (Figure 11 A, B, C and D). These differences were always significant when female mosquitoes fed water alone and those fed $20 \%$ sucrose were compared (Figure $11 \mathrm{~A}, \mathrm{~B}, \mathrm{C}, \mathrm{D})$

\subsubsection{Previtellogenic methoprene application increases stored ovarian lipids}

Previous work indicated that $\mathrm{JH}$ during the resting stage was an important regulator of fecundity by preventing follicular resorption (Clifton and Noriega, 2011). To investigate a possible role for $\mathrm{JH}$ in regulating the biochemical and molecular characteristics of the ovary during the resting stage, hormonal manipulations with methoprene were performed. Methoprene application alters the morphology of the follicle similar to the effect seen with $20 \%$ sucrose (Figures $10 \mathrm{~A}$ and 12A). Oocytes of mosquitoes treated with methoprene appear to contain massive increases in lipid content over acetone controls after staining with oil red-O (Figure 12A). Quantification of ovarian lipids revealed that methoprene application causes a 8-fold increase in lipid content over acetone treatment alone in mosquitoes fed $3 \%$ sucrose $(2760$ pmol. vs. 333 pmol. respectively; Figure 12B; Mann-Whitney test; $p<.01$ ). In mosquitoes fed $20 \%$ sucrose, methoprene treatment causes a similar increase in the lipid content of the oocyte as compared to acetone controls (4180 vs. 702 pmol.; Figure 12B; Mann-Whitney test; $p \leq .05)$. Mosquitoes fed water alone and treated with 
methoprene also show a significant increase in the lipid content of oocytes when compared to acetone controls (1906 vs. 257 pmol.; Figure 12B; Mann-Whitney test; $p \leq .05)$.

\subsubsection{Previtellogenic methoprene application alters expression of genes important for vitellogenesis}

A comparison of the effect of methoprene on the expression of genes important to translation and endocytosis during vitellogenesis (rpL32, AaVgR, AaLpRov, $A a C H C)$ reveals that the effect of methoprene is similar to that of

nutrition (Figures $13 \mathrm{~A}, \mathrm{~B}, \mathrm{C}$ and D). Ribosomal protein L32of the 60 s subunit showed a significant $38 \%$ increase in expression over controls with methoprene treatment (Figure 13A). AaLpRov also increased significantly $76 \%$ compared to acetone controls (Figure 13B). $\mathrm{AaCHC}$ increased significantly $51 \%$ compared to acetone controls (Figure 13D) and although not statistically significant due to the high variability in previtellogenic expression observed with this receptor, $A a V g R$ increased $61 \%$ on average (Figure 13C; $p=.09$; unpaired t-test). In each case, the effect of methoprene mimicked the effect of a $20 \%$ sucrose feeding regime.

\subsubsection{The fate of follicles after a blood meal is affected by previtellogenic nutrition}

To discount the possibility that reproductive decisions made during the resting stage can be rescued by a blood meal and confirm that previtellogenic nutrition (and its associated effects on gene expression and ovarian lipid content) affects the rate of resorption after a blood meal, the dry mass of a blood meal was determined for mosquitoes fed water alone, $3 \%$ sucrose and $20 \%$ sucrose 
(Figure 14). As sugar concentration increased, (and nutritional reserves increased as shown by figure 9B) the mass of the blood meal decreased (Figure 14). Mosquitoes that were maintained on $20 \%$ sucrose had a blood meal mass that was nearly $70 \%$ less than mosquitoes fed water alone (Figure 14). The decrease in dry blood meal mass was statistically significant for each treatment $(p<.001 ;$ unpaired t-test).

To confirm the effect previtellogenic nutrition and hormones have on the fate of follicles after a blood meal, mosquitoes were maintained on $3 \%$ sucrose and $20 \%$ sucrose concentrations and were fed blood. Ovaries from mosquitoes maintained on $3 \%$ sucrose and then examined 24 hours after a blood meal showed a significantly higher rate of follicular resorption (Figures 15A; $p<.001$; unpaired t-test) and those follicles that were not resorbing were smaller on average than follicles from mosquitoes fed $20 \%$ sucrose $(197.8 \mu \mathrm{m}$ vs. $203.2 \mu \mathrm{m}$; $p<.001$; unpaired $\mathrm{t}$-test). Mosquitoes that were fed $20 \%$ sucrose before a blood meal had nearly three times less resorption than those fed $3 \%$ sucrose $(13.6 \%$ vs. $4.8 \%$; Figure $15 \mathrm{~A}$ ). Mosquitoes fed $20 \%$ sucrose also laid on average 10.4 more eggs per female (117.3 vs. 106.9; $p<.05$; unpaired t-test; Figure 15B). The effect of previtellogenic $\mathrm{JH}$ III treatment on vitellogenic resorption is similar to the effect of offering high sucrose concentrations. Mosquitoes fed $3 \%$ sucrose and treated with $\mathrm{JH}$ III had $40 \%$ less resorption 24 hours after a blood meal (12.6\%vs. 7.6\%; Figure 15C). Mosquitoes treated with $\mathrm{JH}$ III also laid on average 11.2 more eggs per female than those mosquitoes treated with acetone alone (104.4 vs. 93.2; $\mathrm{P}<.01$; unpaired t-test; Figure 15D). However, the proportion of eggs 
laid that successfully hatched was not significantly altered by either JHIII treatment or feeding $20 \%$ sucrose over controls (results not shown).

\subsection{Discussion}

\subsubsection{Defining oocyte and follicle quality}

Central to any hypothesis that relies on the differential survival of oocytes during oogenesis is the idea that oocytes vary in their inherent ability to develop and so exist as part of a heterogeneous population. Therefore, the "quality" of an oocyte is likely to determine its potential for further development (reviewed in Gilchrist et al., 2008). While oocyte quality may mean different things depending on the observational context as well as the specific developmental features of an organism, Thomson et al., define oocyte quality broadly as "...[the] intrinsic genetic, epigenetic and cytoplasmic characteristics required for the completion of development and capability of producing a normal offspring" (2010). We examined likely indicators of a follicles ability to complete vitellogenic development (i.e., "quality") such as: oocyte neutral lipid content, lipophorin receptor expression (AaLpRov) (Cheon et al., 2001; Seo et al., 2003; Sun et al., 2000), vitellogenin receptor expression (AaVgR) (Sappington et al., 1996; Cho and Raikhel, 2001), heavy-chain clathrin expression ( $\mathrm{AaCHC}$ ) (Kokoza and Raikhel, 1997; Kokoza et al., 1997) as well as 60s ribosomal protein expression subunit L32 (rpL32). These markers of mosquito oocyte quality, while certainly not meant to be an exhaustive inventory, begin to illustrate how previtellogenic nutrition and $\mathrm{JH}$ synthesis can affect vitellogenic resorption by altering an oocyte's “...intrinsic developmental potential" (Gilchrist et al., 2008). Each of 
these indicators of follicle quality was altered in response to nearly all of the nutritional and hormonal manipulations conducted on previtellogenic mosquitoes. Furthermore, these same nutritional and hormonal manipulations clearly altered the rate of resorption after a blood meal as well as final egg output (Figures 15 $A, B, C$ and $D)$. The biochemical and transcriptional changes that occur in response to manipulations of previtellogenic nutritional and hormonal status (Figures 10, 11, 12 and 13) strongly suggests that the rate of resorption after a blood meal is dependent on the quality of follicles beginning vitellogenesis and also that the quality of follicles is mediated through $\mathrm{JH}$ in response to nutrition. These results also suggest that the intrinsic development potential of a follicle (i.e., follicle quality) can be defined in $A$. aegypti by examining aspects of oocyte function that are likely to be important during vitellogenesis.

\subsubsection{Oocyte lipids can be altered by previtellogenic nutrition as well as hormonal manipulation}

Previtellogenic oocyte lipids may play an important and overlooked role in the reproductive physiology of $A$. aegypti. To our knowledge, only a few studies have been conducted that include any information about previtellogenic ovarian lipids. Tadkowski and Jones suggested that all lipid yolk contained within the oocyte is synthesized within the oocyte both before and after a blood meal (1978). Troy et al., concluded that the principal previtellogenic ovarian lipids are hydrocarbons and fatty esters (1975). However, both of these results were challenged in later work by Zeigler who found that while the ovary can synthesize and store triglycerides and phospholipids, they make up only $1 / 1000^{\text {th }}$ of the final 
lipid content of mature eggs under in vitro culturing conditions (1997). Zeigler and Ibrahim (2001) later described the amount of lipid stored in previtellogenic ovaries as a "trace". To our knowledge, all previous studies have been specifically focused on the origins of the microgram quantities of lipids transferred to eggs after a blood meal and do not describe any potential role for previtellogenic oocyte lipids. The small quantity of previtellogenic triglycerides we examined in the follicles of the ovary are possibly the "minor" or "trace" proportion capable of being synthesized by the ovary itself as described by Zeigler and Ibrahim (2001). However, viewing previtellogenic oocyte-derived lipids as an interchangeable proportion of final egg lipid reserves is probably incorrect and overly simplistic.

The presence of stored lipids in oocytes is widespread among animals, and has been correlated with oocyte "quality" and reproductive success (Nagano et al., 2006a; 2006b; Angel-Dapa, 2010). The exact role of stored previtellogenic lipids in mosquito oocytes is unknown but current evidence suggests that oocyte lipids, at least in those vertebrate animals which have been studied, are an important substrate for energy production in oocytes (Sturmey and Leese, 2003; Ferguson and Leese, 2006; Reviewed in Sturmey et al., 2009). Furthermore, the composition of lipids synthesized or stored by oocytes is generally unique and enriched in specific saturated fatty acids and likely reflects the "...highly specialized requirements" of an oocyte (Homa et al., 1986; Matorras et al., 1998). Similar to vertebrate organisms, Zeigler's work with ovarian lipid synthesis in $A$. aegypti shows that lipids synthesized in the mosquito ovary are also specifically 
enriched in saturated fatty acids (1997). While the exact purpose of stored oocyte lipids and fatty acid enrichment is unclear, the work presented here in conjunction with existing literature does suggest that previtellogenic lipids synthesized and stored in the oocyte have a unique reproductive function which may not be the same as lipids derived from the fat body, which do not show saturated fatty acid enrichment (Zeigler, 1997).

The period after a blood meal in mosquitoes is marked by intense vitellogenin receptor, lipophorin receptor and heavy-chain clathrin synthesis by the oocyte and nurse cells (Sappington et al., 1996; Kokoza and Raikhel, 1997; Kokoza et al., 1997; Sun et al., 2000; Cheon et al., 2001; Cho and Raikhel, 2001; Seo et al., 2003), as well as massive endocytosis of yolk components (Reviewed in Dhadialla and Raikhel, 1992). Since endocytosis and protein synthesis are both energy-dependent processes, it is not unreasonable to suggest that a pool of previtellogenic oocyte lipids supports the energetic demands of the growing oocyte during at least the beginning stages of vitellogenesis. This pool of oocyte lipids is clearly affected by methoprene (Figures $12 \mathrm{~A}$ and $\mathrm{B}$ ) as well as nutritional status (Figures $10 \mathrm{~A}$ and $\mathrm{B}$ ) during the resting stage and may provide the energetic basis for an endocytotic "competition" between follicles for yolk components during vitellogenesis as suggested by Bell and Bohm (1975) as well as provide the basis for understanding why one follicle resorbs besides another which is developing (Lea et al., 1978; Clements and Boocock, 1984). While we did not specifically investigate the composition of ovarian lipids, the total quantity of neutral lipids stored in the ovary seems to reflect overall nutritional reserves 
(Figures 10A, B). This result suggests that the pool of ovary lipids is dynamically regulated and integral to the coordination of fecundity with previtellogenic nutrition during vitellogenesis.

\subsubsection{Previtellogenic expression of the endocytotic complex can be altered by nutrition and methoprene application}

We also examined the previtellogenic mRNA levels of three proteins integral to the rapid receptor-mediated endocytosis that occurs during vitellogenesis. Much previous work has demonstrated that the post-eclosion formation of an endocytotic complex is dependent on a peak of $\mathrm{JH}$ synthesis (Raikhel and Lea, 1985) and that the endocytotic complex is composed of vitellogenin receptors ( $A a V g R)$ (Sappington et al., 1996; Cho and Raikhel, 2001), lipophorin receptors (AaLpRov), (Cheon et al., 2001; Seo et al., 2003; Sun et al., 2000), as well as heavy-chain clathrin ( $\mathrm{AaCHC}$ ) (Kokoza and Raikhel, 1997; Kokoza et al., 1997). Since the initial formation of an endocytotic complex in mosquito follicles is dependent on $\mathrm{JH}$, and stored ovarian lipids were clearly altered during the previtellogenic resting stage in response to methoprene (Figures 12A, B), we hypothesized that the transcription and accumulation of mRNA's for these genes would also be similarly modulated in response to hormones and nutrition. As our results suggest, $\mathrm{JH}$ not only induces the formation of an endocytotic complex, but may also maintain the expression of the components of the endocytotic complex in response to previtellogenic nutritional status. 
Although limited evidence exists, Cho and Raikhel have suggested that transcripts of genes important to vitellogenesis are made by the nurse cells, transferred to the oocytes, and tend to accumulate during the previtellogenic resting stage (2001). In situ hybridizations performed in previous studies for each of these endocytotic genes demonstrates substantial mRNA accumulation in the cytoplasm of the oocyte (Kokoza and Raikhel, 1997; Cho and Raikhel 2001; Cheon et al., 2001; Seo et al., 2003). Presumably, the large-scale translation of these components to support endocytosis and vitellogenesis would then depend on a blood meal (stored oocyte lipids during this time would be especially useful in providing the energy for rapid up-regulation of the endocytotic complex) (Kokoza and Raikhel, 1997). Studies in vertebrate and invertebrate animals have indicated that in addition to neutral lipids, storage of mRNA and protein components necessary for development by the oocyte is a ubiquitous and vitally important determinant of oocyte quality that is often correlated with an oocyte's likelihood of successful fertilization and development (Brandhorst, 1980; Reviewed in Eichenlaub-Ritter and Peschke, 2002; Leoni et al., 2006; reviewed in Wrenzycki and Niemann, 2007; reviewed in Mermillod et al., 2008).

In addition to endocytotic components, we also examined the abundance of mRNA's for the protein L32 of the ribosomal 60s subunit. In each treatment, the abundance of rpL32 mRNA behaved similarly to genes necessary for vitellogenesis. The abundance of ribosomal mRNA's in the ovary is likely related to the developmental capacity of the oocyte and reflects the capacity of the oocyte to synthesize proteins after a blood meal or during embryogenesis. In 
those animals which have been studied, the majority of ribosomal proteins (Bachvarova and Deleon, 1977) and ribosomal mRNA's (Burkholder et al., 1971; Reviewed in Eichenlaub-Ritter and Peschke, 2002) are stored in the oocyte for later use. While we did not study the function of the accumulations of ribosomal mRNA's it seems likely that the translational capacity of the oocyte would be coregulated to match available energy (oocyte lipid stores) and available template material (mRNA's related to vitellogenesis).

In this study we attempted only to quantify the abundance of mRNA's for genes likely to be important after a blood meal in order to define and characterize the morphological changes we observed in previtellogenic ovaries (Figure 9A). Because we only examined mRNA abundance on a per follicle basis we cannot be certain how translational events before or after a blood meal affect the expression of these endocytotic components. Despite this, the dependence of neutral lipids (Figures 10 and 12) and endocytotic mRNA's (Figures 11 and 13) on previtellogenic nutrition and hormones does suggest that a relationship between the pattern of accumulation of endocytotic mRNA's and a follicle's fate does exist. While we did not specifically explore the individual differences between follicles of the same ovary, it is likely that differences in mRNA accumulation do exist between follicles of the same ovary which would provide the basis for a vitellogenic "competition" for yolk components.

\subsubsection{Low reserve mosquitoes take more blood but develop fewer eggs}

A major factor that must be included in any explanation of vitellogenic resorption in mosquitoes is the role of a blood meal. Sugar feeding has been 
shown to affect blood meal size such that recently sugar fed mosquitoes with full crops can experience reductions in fecundity as a consequence of blood meal volume limitations in the abdomen (Mostowy and Foster, 2004). Additionally, a proportion of any reduction in fecundity can be attributed to previtellogenic resorption and is therefore completely independent of a blood meal (Figure 17; Clifton and Noriega, 2011). Despite crop-volume effects and previtellogenic resorption, the relationship between fecundity, blood meal size and previtellogenic nutrition still appears to be counterintuitive in A. aegypti, primarily because large blood meals cannot rescue low reserve mosquitoes even when crop volume is controlled (Mostowy and Foster, 2004).

To explain this incongruence, we would like to suggest that stored previtellogenic fat body reserves are not only important for direct incorporation into eggs, but may also be critically important in providing the energy to digest a blood meal and manufacture yolk proteins. The digestion of blood likely requires significant energy as evidenced by the increased rate of respiration after a blood meal (Gray and Bradley, 2003; Sarfati et al., 2005). Work by Zhou et al., (2004a) showed that $\sim 29 \%$ of the blood meal is excreted as waste and $\sim 43 \%$ is used as energy suggesting that there is some limit to the amount of nutrition that can actually be recovered from a blood meal for use in eggs. The same work has also shown that up to $\sim 50 \%$ of mobilized fat body lipid reserves are utilized as energy after a blood meal (Zhou et al., 2004a). Therefore, the extent of previtellogenic nutritional reserves may partially dictate how much of the nutrition contained within a blood meal is ultimately recoverable and convertible into yolk 
components. The ubiquity of resorption after a blood meal in mosquitoes of all nutritional conditions strongly suggests that blood can never prevent all resorption and that yolk components are probably always in short supply during vitellogenesis (Lea et al., 1978; Clements and Boocock, 1984; Nayar and Sauerman, 1975; Mostowy and Foster 2004). Taken together with the multitude of studies that have shown a clear relationship between the magnitude of resorption and blood meal size (Woke et al. 1956, Colless and Chellapah 1960, Jalil 1974; Lea et al., 1978; Mostowy and Foster, 2004), a limit to the nutrition that can be derived from a blood meal would explain the high levels of vitellogenic resorption seen in low reserve mosquitoes despite the acquisition of a large blood meal. In this context, previtellogenic lipid reserves may be doubly important by: (1) determining the extent of utilization of a blood meal and/or the extent of synthesis of yolk proteins and (2) for direct incorporation into follicles during oogenesis. Although we did not specifically explore how previtellogenic nutrition affects blood feeding behavior or blood meal utilization, it is clear that blood feeding behavior, previtellogenic nutritional status and fecundity are intricately related.

\subsubsection{A model for understanding the coordination of reproductive output with nutrition in the anautogenic mosquito}

The study presented here attempts to answer two lingering questions posed in the literature about the regulation of resorption and the reproductive physiology of anautogenic mosquitoes: 1) How does the mosquito determine the extent of resorption (i.e. coordinate fecundity) based on blood meal size and 
previtellogenic reserves? (Lea et al., 1978; Browne, 2001; Mostowy and Foster, 2006) and also 2) how does the mosquito decide which follicles to resorb? (Lea et al.,1978; Clements and Boocock, 1984). When the results of this study are integrated with existing literature, a regulatory scheme emerges that can potentially answer these questions and provide a conceptual framework with which to understand the coordination of reproductive output with nutrition in anautogenic mosquitoes (Figure 16).

The work presented here suggests that $\mathrm{JH}$ during the resting stage regulates the physiology of a presumably heterogeneous population of previtellogenic follicles in response to previtellogenic nutrition. After a blood meal, all primary follicles begin depositing yolk components in the oocyte (Clements and Boocock, 1984; Lea et al., 1978). As a "competition" for these yolk components commences (as suggested by Bell and Bohm, 1975), those follicles which are lagging in development or otherwise unable to compete are ultimately resorbed in favor of those follicles which possess a better ability to develop (Figure 16). The best support for such a competition comes from experiments by Schlaeger et al., (1974) and confirmed in our laboratory in which high doses of 20-hydroxyecdysone injected into previtellogenic mosquitoes caused all follicles to begin depositing yolk, only later to be resorbed ( $\sim 8$ hours) in favor of just a few highly developed follicles comprised solely of whatever endogenous nutritional reserves were available for incorporation at the time of ecdysteroid treatment (results not shown). 
Although ecdysteroid injection induced massive resorption and the development of a few eggs, other work with Caenorhabditis elegans showed the consequences of preventing apoptosis and resorption in oocytes. When apoptosis was prevented, remaining oocytes showed smaller sizes and lower viability implying that the pool of reproductive resources was limited and dividing this pool among more oocytes had negative consequences. The authors concluded that a "competition" for resources among aging oocytes maintains oocyte quality by resorbing those of inferior quality (Andux and Ellis, 2008). In Nauphoeta cinerea, the same coordination between the pool of reproductive resources and egg number was shown when resources recovered from resorbing follicles were reinvested into the remaining follicles and were specifically not returned to the pool of somatic resources (Barrett et al., 2009). In Periplaneta americana, this phenomenon was taken a step further when yolk proteins from resorbing follicles were shown to be released into the haemolyph intact and therefore readily available to developing follicles (Bell, 1971). To our knowledge, Bell's (1971) results have not been repeated in other insects. Although we did not specifically explore the fate of the resources recovered from resorbing follicles or the effects of blocking resorption, our results do suggest that during oogenesis a competition occurs between follicles, based in some part, on the intrinsic quality factors described here.

In addition to initiating further development, it is also likely that ecdysteroids provide the hormonal stimulus for resorption in developmentally inferior follicles (Figure 16). Previous work has shown that an integral 
component of programmed cell death, the effector caspase AeDronc, is most highly expressed during pulses of ecdysteroids, especially during metamorphosis (Cooper et al., 2007). In the adult mosquito ovary, 20-hydroxyecdysone treatment causes a nearly 3 -fold increase in AeDronc transcription (Cooper et al., 2007). In Drosophila melanogaster, ecdysteroid treatment clearly increases ovarian apoptosis and resorption (Söller et al., 1999; Terashima et al., 2005). Although ecdysteroid treatments induce apoptosis and resorption in insects with sequential egg development (thereby directly controlling reproductive output), it has been shown previously by Uchida et al., (1998) in Culex pipiens pipiens and reaffirmed for $A$. aegypti (Uchida and Moribayashi, 2002) that the ecdysteroid titer itself likely does not regulate the determination of egg number during vitellogenic development in insects with synchronous egg development (Browne, 2001). The failure of ecdysteroid titers to determine egg number suggests that a threshold concentration of ecdysteriods exists that induces resorption in sensitive follicles and that $\mathrm{JH}$ during previtellogenic development participates in determining the sensitivity of follicles to ecdysteriods. The reduction in resorption after a blood meal in those mosquitoes treated with $\mathrm{JH}$ III (Figure $15 \mathrm{C}$ ) during the resting stage further supports a role for $\mathrm{JH}$ during vitellogenic resorption.

A similar regulatory scheme has been described to explain follicular resorption in $D$. melanogaster by Terashima et al., (2005). In D. melanogaster, starvation causes a rapid increase in ecdysteroid titer followed by increased resorption of follicles (Terashima et al., 2005). Since some ecdysteroid titer is required in this species for normal oogenesis, an activating threshold can explain 
how ecdysteriods can be dually important for oogenesis as well as resorption (Terashima et al., 2005). The co-occurrence of vitellogenic resorption (Clements and Boocock, 1984) with high ecdysteroid titers (Greenplate et al., 1985) in A. aegypti also suggests that the onset of vitellogenic resorption in susceptible follicles is probably dictated by an ecdysteroid threshold after a blood meal. A regulatory scheme that relies on the differential sensitivity of follicles to a "threshold" of ecdysteroids would explain how one follicle resorbs beside another which is developing (Figure 16). Despite the ability of $\mathrm{JH}$ and nutrition to alter the average quality of follicles, the exact mechanism that confers differential sensitivity to ecdysteriods is still unknown, although our results here suggest it is related to the intrinsic ability of the oocyte to incorporate yolk components and undergo vitellogenic development.

\subsubsection{Conclusions}

In this study we examined likely indicators of a follicles ability to complete vitellogenic development (i.e. "quality") such as: oocyte lipid content, lipophorin receptor expression, vitellogenin receptor expression, heavy chain clathrin expression and ribosomal protein expression. Each of the explored quality markers represents a small cross-section of the possible changes that occur in the oocyte in response to hormones and nutrition. However, a few key details about the regulatory scheme that the work here suggests remain unanswered. (1) How does previtellogenic nutritional status alter blood feeding behavior? (2) Does the extent of vitellogenic development within an individual follicle during the period of high ecdysteroid titer determine the likelihood of resorption? (3) How 
does the extent of vitellogenic development within a follicle determine the sensitivity of the follicle to ecdysteroids and the likelihood of resorption?

By modulating the follicles response to the hormonal and nutritional environment after a blood meal, JH seems to integrate previtellogenic nutritional information with blood meal derived nutrition to determine reproductive output (Figure 17). This regulatory scheme, by expanding a role for $\mathrm{JH}$ to include postblood meal resorption, illustrates the endocrinological compromises that underlay the peculiarities of an anautogenic life history strategy such as a previtellogenic resting stage, sugar feeding, synchronous egg development, and hematophagy.

\subsection{Acknowledgments}

We thank Dr. Marcela Nouzova for rearing mosquitoes as well as Dr. Marcela Nouzova, Dr. Crisalejandra Rivera-Perez and Mario Perez for critical reading of the manuscript. This work was supported by NIH Grant No. Al 45545 to F.G.N. 


\section{$r p L 32$}

rpL32 Forward: 5' CCAAGCCAGATTCCTAACATTCAAA 3'

rpL32 Reverse: 5' TGCCCTGTCCGCAACC 3'

rpL32 Probe: 5' CTTGCAATCATTACCACAGCAC 3'

AaLpRov

AaLpRov Forward 5'GGTAAAACTGCGTCATCGTCTTC 3'

AaLpRov Reverse: 5'GTGCAAACTGTTAGCGAACGA 3'

AaLpRov Probe: 5'TCGATGAGGAAGAGTTATTG 3'

AaCHC

AaCHC Forward: 5' CGATTGTTTCGCGGCATGTTTA 3'

AaCHC Reverse: 5' CCATGCCAGCTCCAAGATGATA 3'

AaCHC Probe: 5' TTCCAGTGCTACGACCTC 3'

AaVgR

AaVgR Forward: 5'GCAAAATAGTGGTGCATGATCGA 3'

AaVgR Reverse: 5'GGCTGTAGACCGGAGTGATAGAT 3'

AaVgR Probe: 5'ATGAACATCGAAAATCTG 3'

Table 1: Primer probes sequences for qPCR (exon borders in bold). 


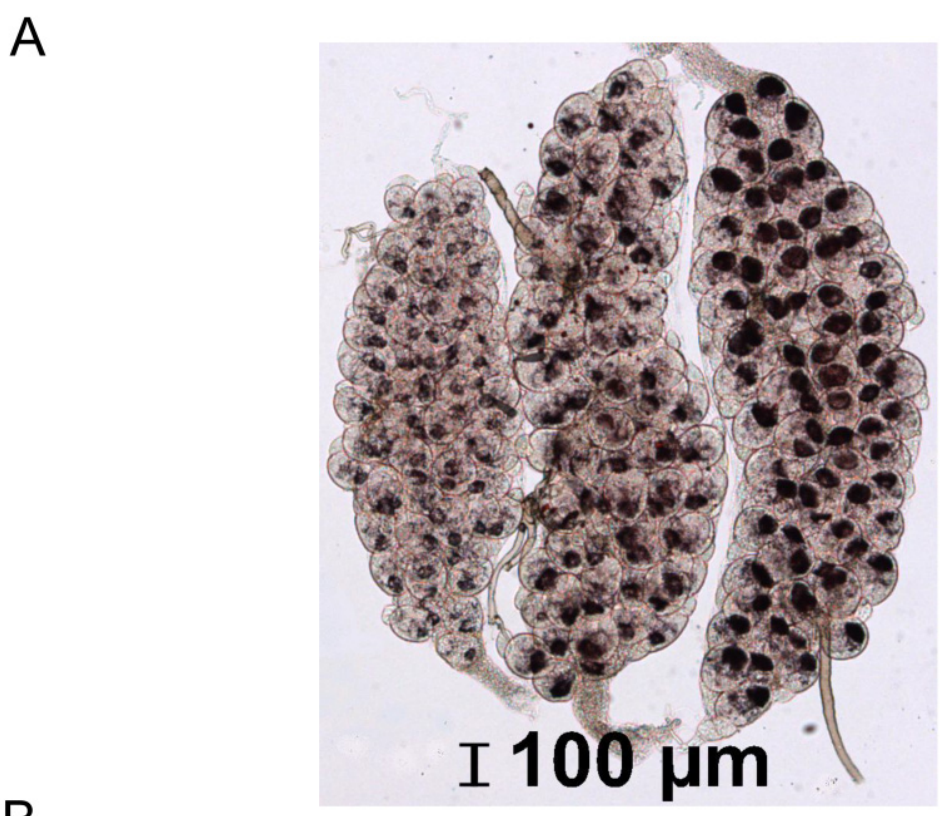

B

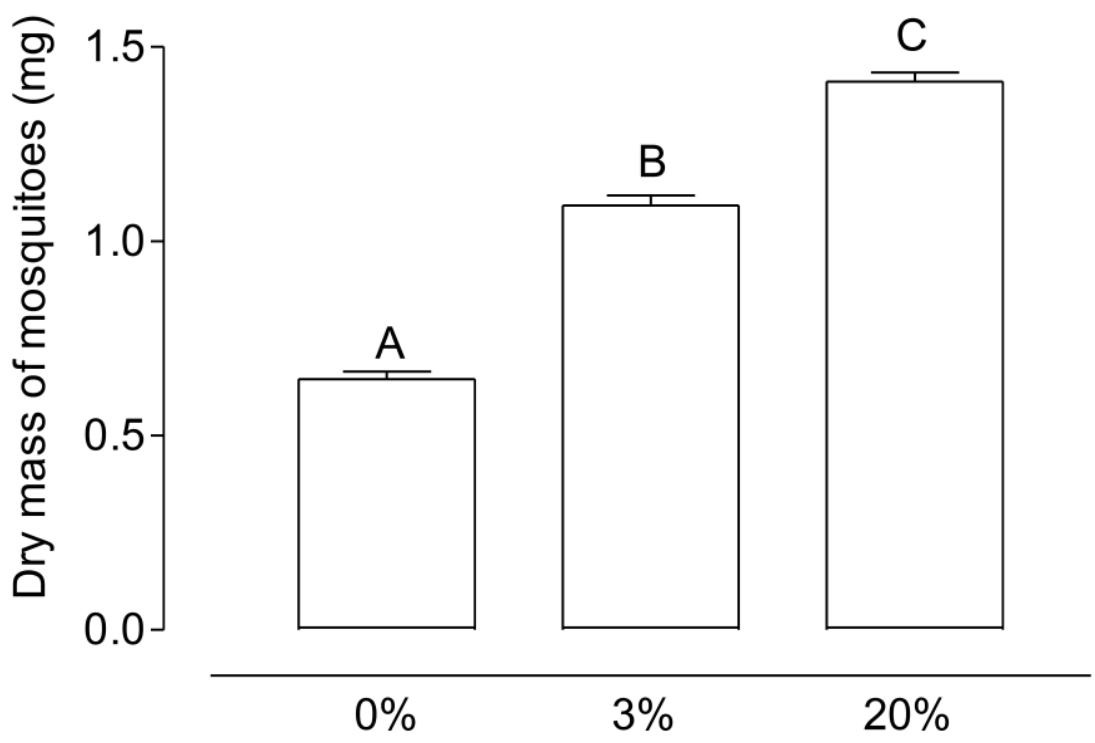

Figure 9: Previtellogenic ovary morphology reflects previtellogenic nutritional reserves. (A) Representative images of single ovaries from mosquitoes maintained with water alone, $3 \%$ sucrose and $20 \%$ sucrose. (B) Dry mass of mosquitoes maintained with water alone, $3 \%$ sucrose and $20 \%$ sucrose (Mean \pm SEM of $\geq 20$ mosquitoes per treatment). The dry mass of mosquitoes significantly increases as the concentration of sucrose offered increases (unpaired t-test; Treatments with different letters denote statistical significance at $p \leq .001)$. 
A

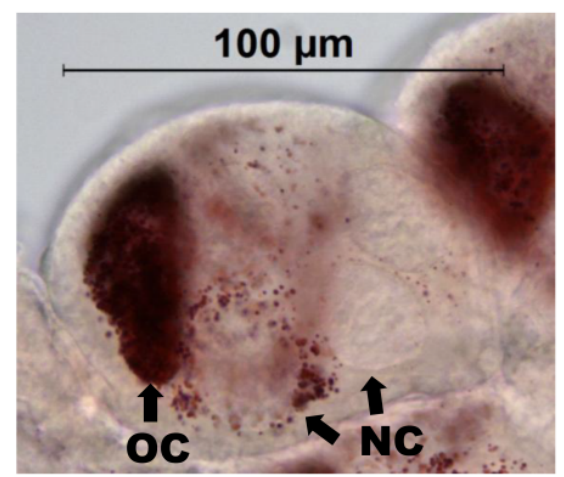

$3 \%$ sucrose

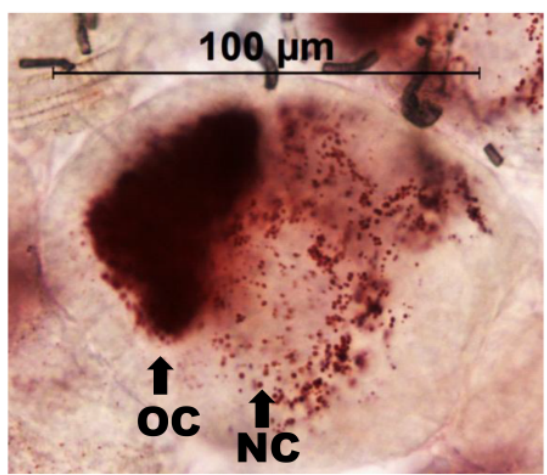

$20 \%$ sucrose

B

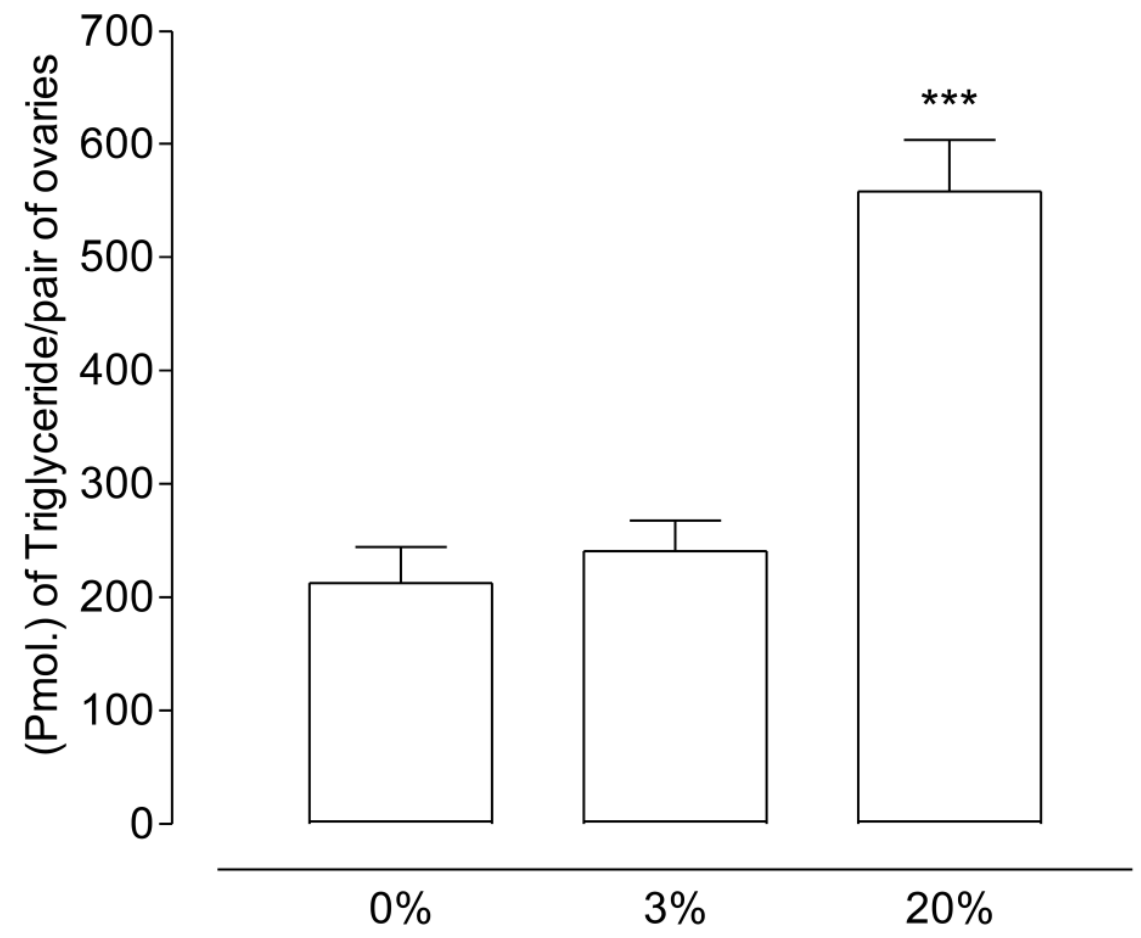

Figure 10: Lipid accumulation in the follicle increases in mosquitoes offered $20 \%$ sucrose. (A) Representative images of a single follicle from mosquitoes maintained with $3 \%$ sucrose and $20 \%$ sucrose. Lipids accumulate predominantly in the oocyte (OC) and proximal nurse cells (NC) as indicated by oil red-o staining of neutral lipids. (B) The quantity of mono- di- and triglycerides in the ovaries significantly increases when mosquitoes are fed $20 \%$ sucrose as compared to those fed water alone $(0 \%)$ or $3 \%$ sucrose solution (3\%). (Mean \pm SEM of at least 3 groups of 5 ovaries per treatment collected for at least three independent experiments; Mann-Whitney U-test; ${ }^{* * *} \mathrm{p} \leq .001$ ). 


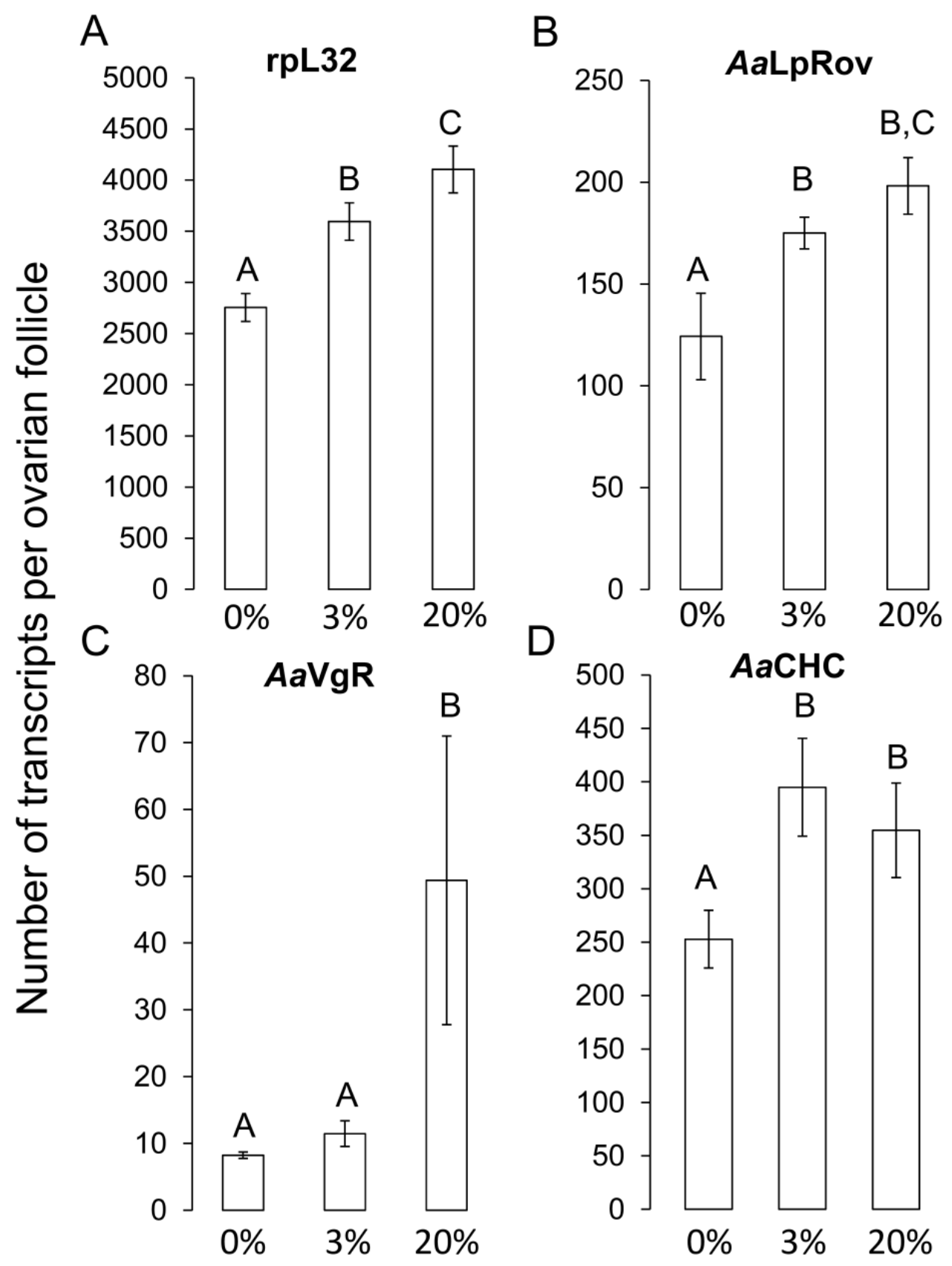

Figure 11: Abundance of mRNA transcripts for $(A)$ ribosomal 60s protein rpL32, $(B)$ lipophorin receptor AaLpRov, (C) vitellogenin receptor (AaVgR) and (D) Heavy-Chain Clathrin $\mathrm{AaCHC}$ in the ovaries of mosquitoes fed water only $(0 \%), 3 \%$ sucrose solution $(3 \%)$, or $20 \%$ sucrose solution $(20 \%)$. (Mean \pm SEM of 5 pairs of ovaries collected for 4 replicates per treatment; unpaired t-test; Treatments with different letters denote statistical significance at $p \leq .05)$. 


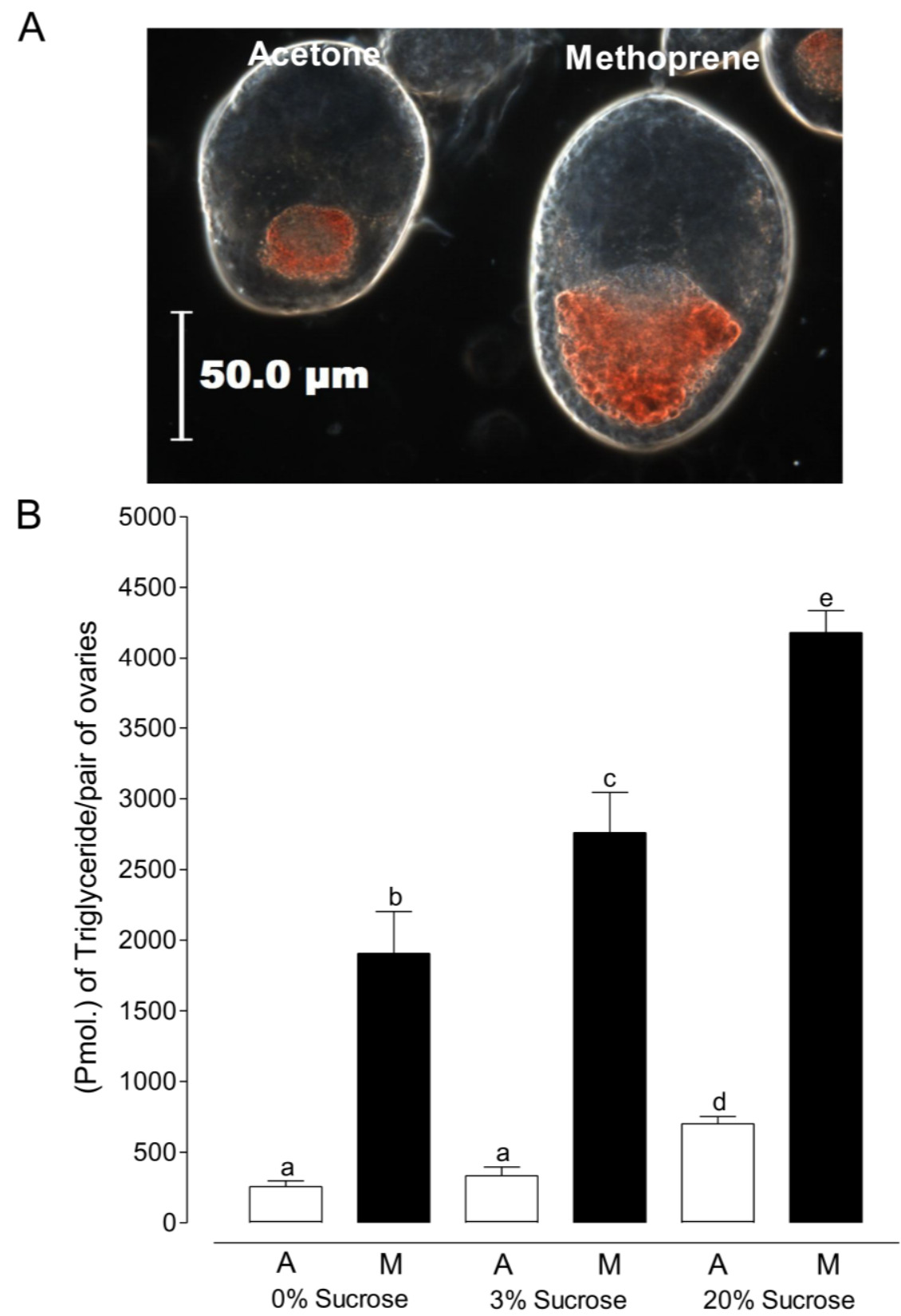

Figure 12: Lipid accumulation in the follicle increases when mosquitoes are treated with methoprene. (A) Representative images of a single follicle from mosquitoes maintained with 3\% sucrose and treated with methoprene dissolved in acetone or acetone alone. Lipids accumulate predominantly in the oocyte and proximal nurse cells as indicated by oil red-o staining of neutral lipids. (B) The quantity of mono- diand triglycerides in the ovaries increases dramatically when mosquitoes fed water alone $(0 \%), 3 \%$ or $20 \%$ sucrose are treated with methoprene (Mean \pm SEM of 5 pairs of ovaries collected for at least 4 replicates per treatment; Mann-Whitney U-test; Different letters denote significance $p \leq .05$ ). 


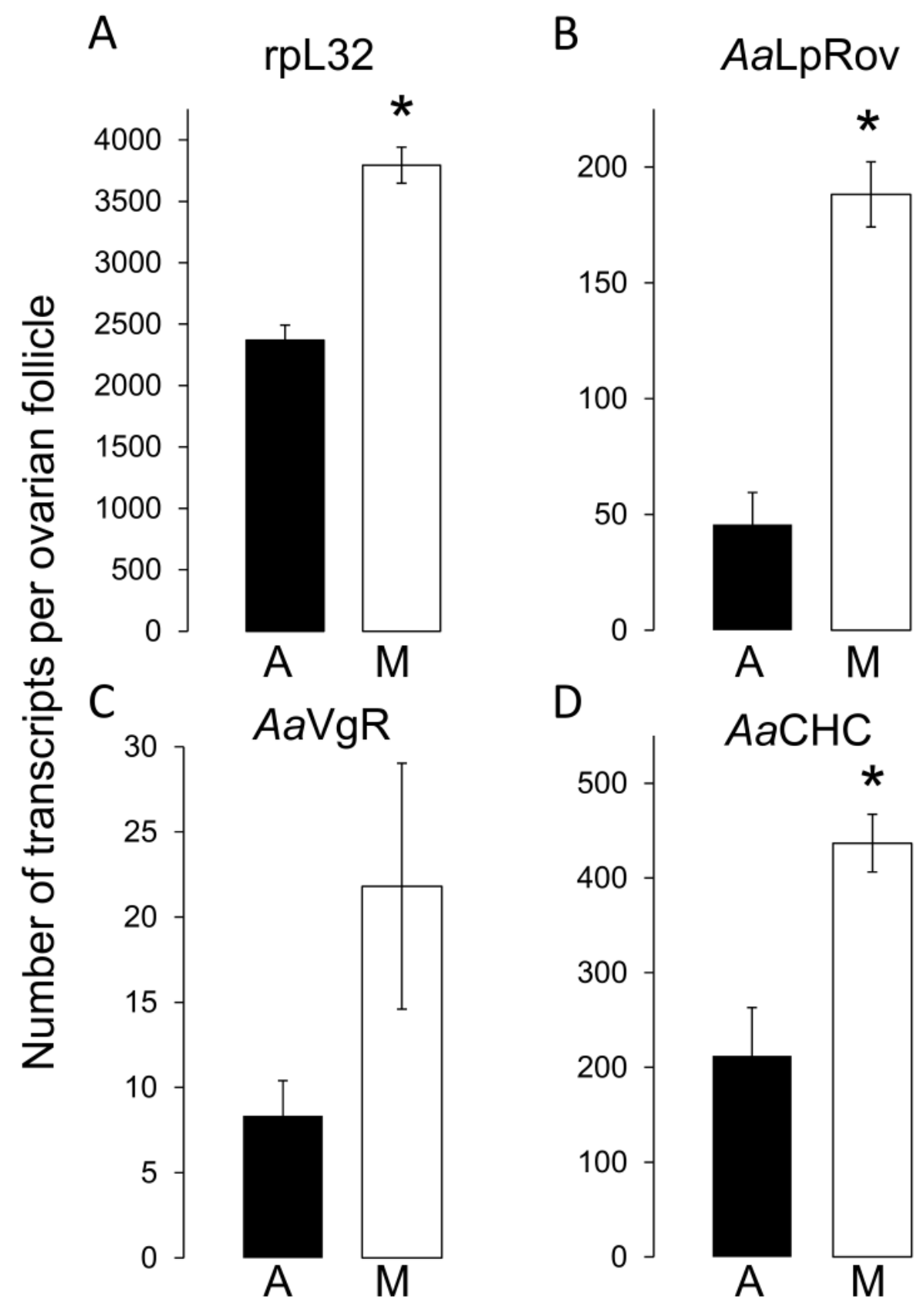

Figure 13: The abundance of mRNA transcripts in the ovaries increases when mosquitoes are treated with methoprene ( $\mathrm{M}=$ methoprene treatment) as compared to controls treated with acetone ( $A=A$ cetone treatment). (A) ribosomal 60 s protein rpL32, (B) the lipophorin receptor (AaLpRov), (C) the vitellogenin receptor, $(A a V g R)$ and (D) Heavy-Chain Clathrin $(A a C H C)$. Different letters denote statistical significance (Mean \pm SEM of 5 pairs of ovaries collected for 4 replicates per treatment; unpaired t-test; $\left.{ }^{*} \mathrm{p} \leq .05\right)$. 


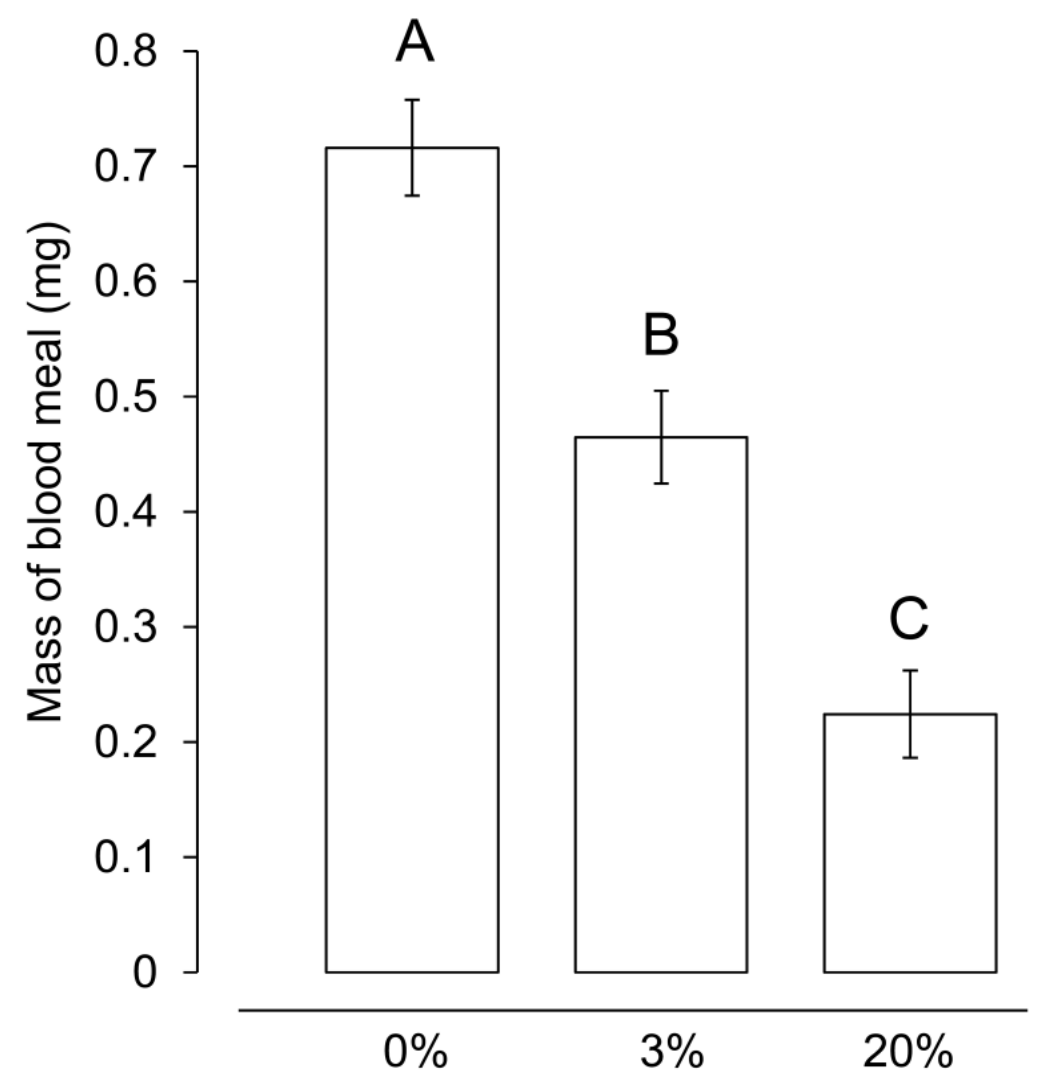

Figure 14: Mosquitoes offered higher concentrations of sucrose take significantly smaller blood meals. (Mean \pm SEM; unpaired t-test; Treatments with different letters denote statistical significance at $p \leq .001)$. 
A

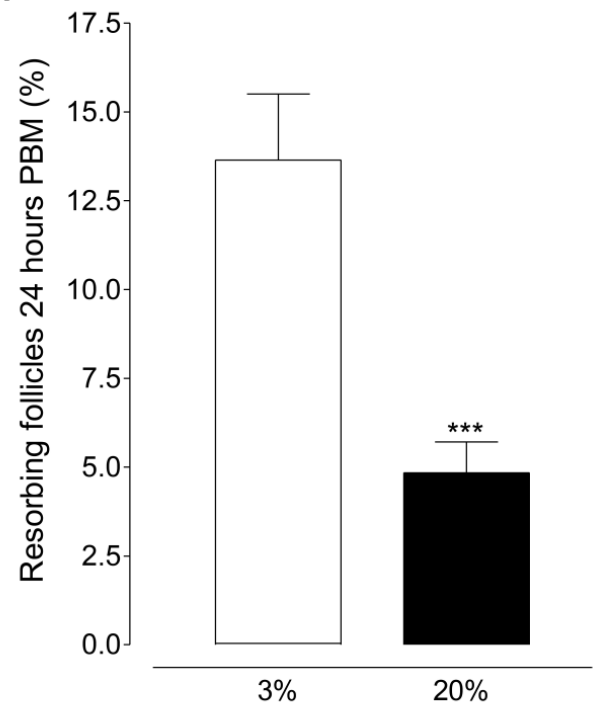

C

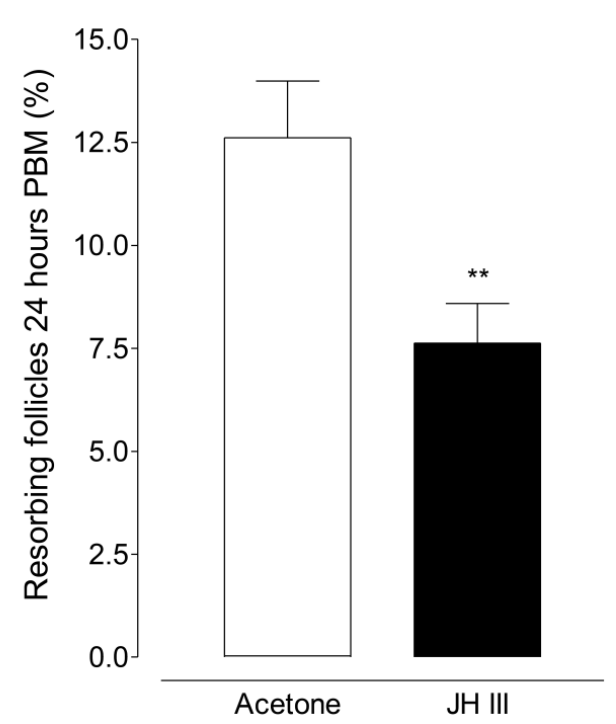

B

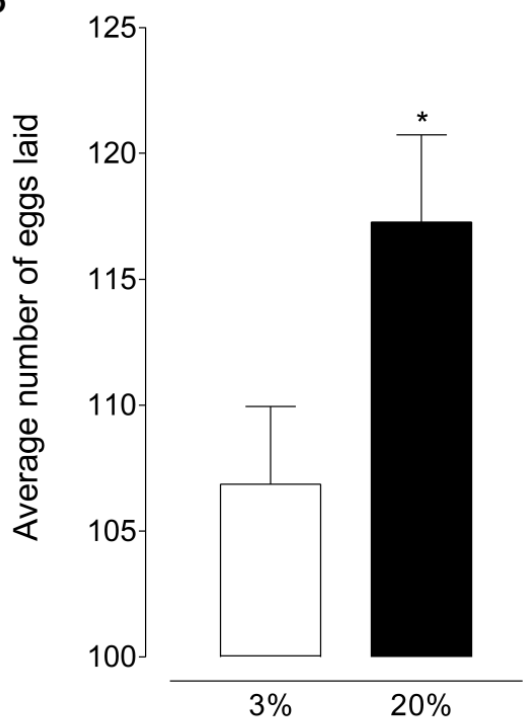

D

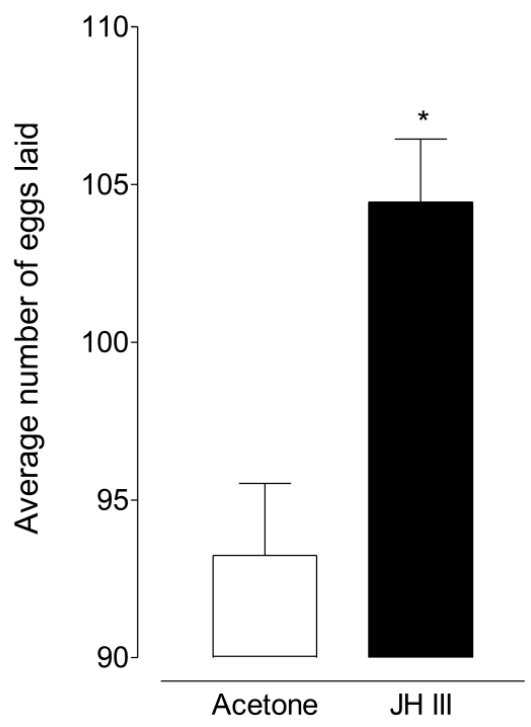

Figure 15: Sugar feeding or hormone treatment during the previtellogenic resting stage alters the rate of resorption 24 hours after a blood meal as well as total egg output. (A) Mosquitoes offered $20 \%$ sucrose show nearly 3 -fold less resorption than mosquitoes offered $3 \%$ sucrose prior to a blood meal (Mean \pm SEM of 10 pairs of ovaries collected in two independent replicates per treatment; Unpaired t-test; $\left.{ }^{* * *} p \leq .001\right)$. (B) Mosquitoes offered $20 \%$ sucrose laid 10.4 more eggs than mosquitoes fed $3 \%$ sucrose (Mean \pm SEM of eggs from 21 females Unpaired t-test; * $p \leq .05$ ). (C) JH III application prior to a blood meal reduces vitellogenic resorption by $40 \%$ (Mean \pm SEM of 15 pairs of ovaries collected in two independent replicates per treatment; Unpaired t-test; ${ }^{* *} p \leq .01$ ). (D) Mosquitoes fed $3 \%$ sucrose and treated with JH III laid 11.2 more eggs than mosquitoes treated with acetone (Mean \pm SEM of eggs from 21 females; Unpaired t-test; $\left.{ }^{*} p \leq .05\right)$. 


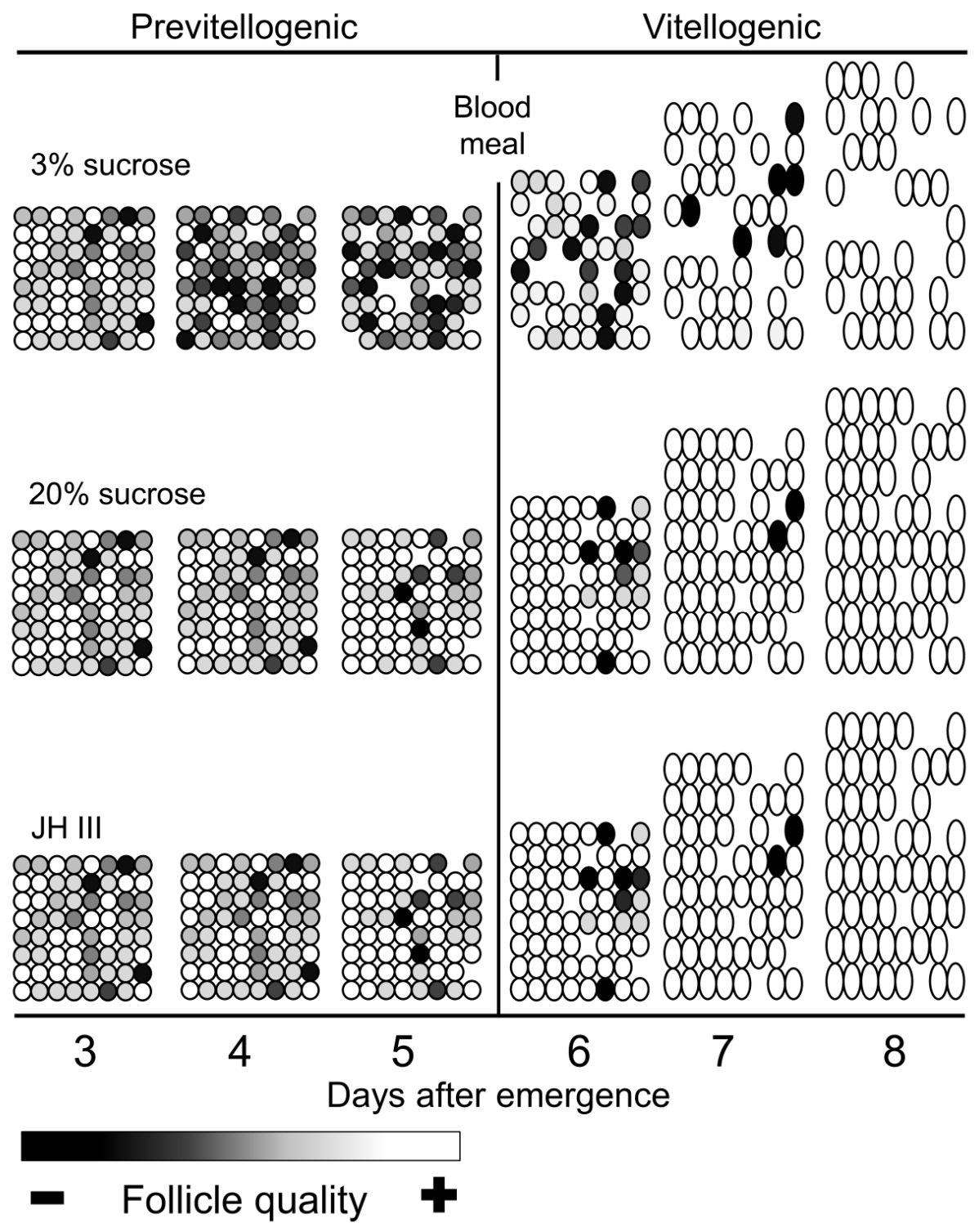

Figure 16: A model that describes the effect of nutrition and hormones during the previtellogenic resting stage on follicular resorption after a blood meal. Extent of grey shading is indicative of follicle quality. Black follicles are being resorbed. Day 3: Initial previtellogenic maturation of the ovarian follicles is completed and a heterogeneous population of follicles exists. Days 4 and 5: Until a blood meal is found, follicles of lesser quality are resorbed and overall fecundity is reduced. Feeding $20 \%$ sucrose or applying $\mathrm{JH}$ III reduces resorption and increases average follicle quality. Day 6: After a blood meal, all remaining follicles begin vitellogenesis and a competition for yolk components. Day 7: As ecdysteroid titers increase and reach a peak, follicles of lower quality and lagging in vitellogenic development are resorbed in favor of those with advanced vitellogenic development. Day 8: A batch of eggs adjusted to match nutritional status during the previtellogenic resting stage and after a blood meal is ready for oviposition. 


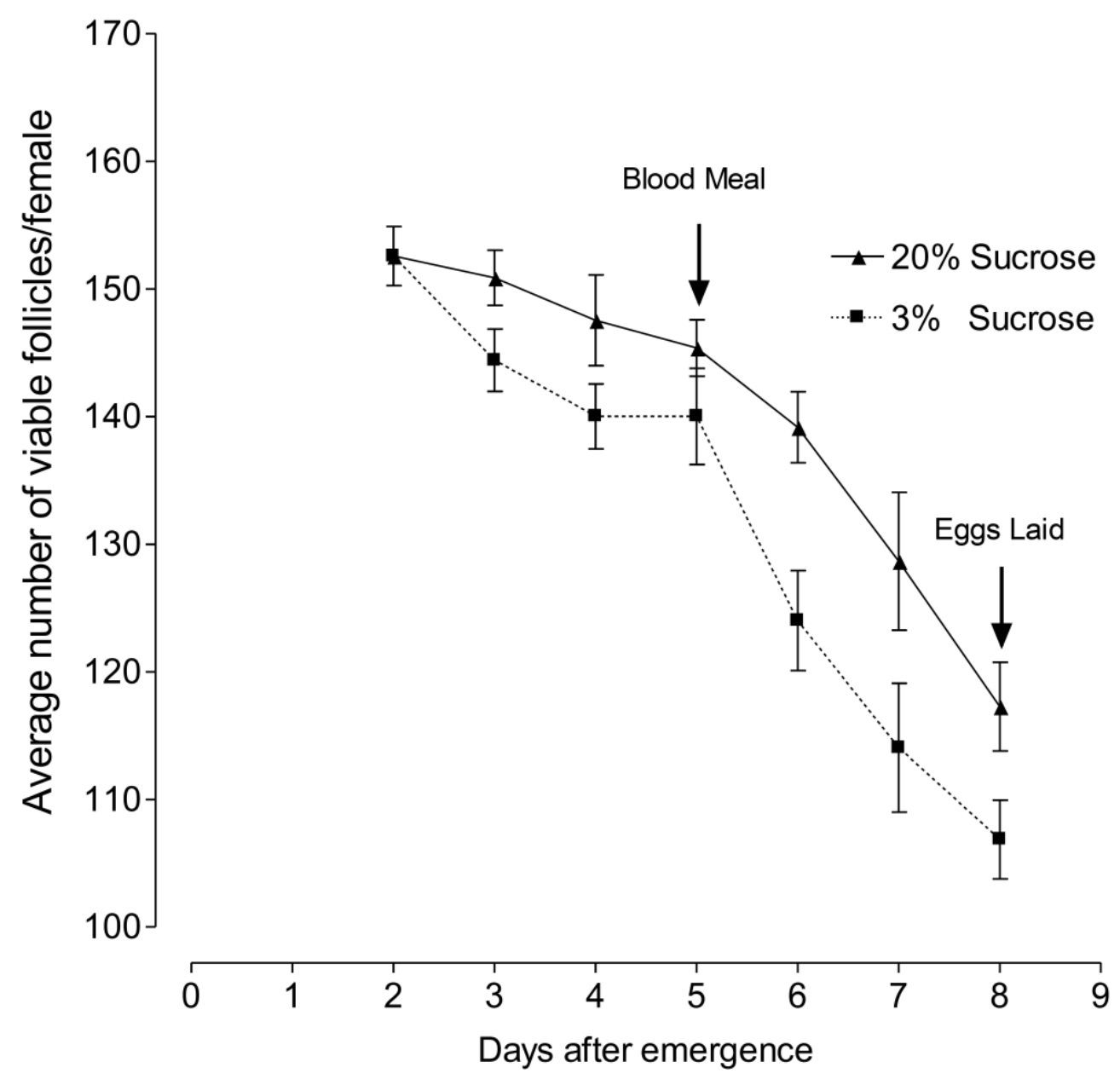

Figure 17: The average number of viable follicles decreases during previtellogenic development and vitellogenic development. 


\subsection{References}

Andux, S., Ellis, R.E. 2008. Apoptosis maintains oocyte quality in aging Caenorhabditis elegans females. Plos Genetics. 4, 1-13.

Angel-Dapa, M.A., Rodríguez-Jaramillo, C., Cáceres-Martínez, C.J., Saucedo, P. E. 2010. Changes in lipid content of oocytes of the penshell Atrina mauraas a criterion of gamete development and quality: A Study of Histochemistry and Digital Image Analysis. Journal of Shellfish Research. 29, 407-413.

Bachvarova, R., De Leon, V. 1977. Stored and polysomal ribosomes of mouse ova. Developmental Biology. 58, 248-254.

Barrett, E.B., Moore, A.J., Moore, P.J. 2009. A potential function for oocyte apoptosis in unmated Nauphoeta cinerea. Physiological Entomology. 34, 272-277.

Bell, W.J. 1971. Starvation-induced oöcyte resorption and yolk protein salvage in Periplaneta americana. Journal of Insect Physiology. 17, 1099-1111.

Burkholder, G.D., Comings, D.E., Okada, T.A. 1971. A storage form of ribosomes in mouse oocytes. Experimental Cell Research. 69, 361-372.

Brandhorst, B.P., 1980. Simultaneous synthesis translation and storage of messenger RNA including histone messenger RNA in sea urchin Lytechinus pictus eggs. Developmental Biology. 79, 139-148.

Briegel, H., Hefti, M., Di Marco, E. 2002. Lipid metabolism during sequential gonotrophic cycles in large and small females of Aedes aegypti. Journal of Insect Physiology. 48, 547-554.

Browne, L.B. 2001. Quantitative aspects of the regulation of ovarian development in selected anautogenous Diptera: Integration of endocrinology and nutrition. Entomologia Experimentalis et Applicata. 100, 137-149.

Bell, W.J., Bohm, M.K. 1975. Oosorption in insects. Biological Reviews 50, 373396. 
Cheon, H.M., Seo S.J., Sun J.X., Sappington T.W., Raikhel A.S. 2001. Molecular characterization of the VLDL receptor homolog mediating binding of lipophorin in oocyte of the mosquito Aedes aegypti. Insect Biochemistry and Molecular Biology. 31, 753-60.

Cho, W., Tsao, S., Hays, A.R., Walter, R., Chen, J., Snigirevskaya, E., Raikhel A.S. 1999. Mosquito cathepsin B-like protease involved in embryonic degradation of vitellin is produced as a latent extraovarian precursor. Journal of Biological Chemistry. 274, 13311-13321.

Cho, W.L., Deitsch, K.W., Raikhel, A.S. 1991. An extraovarian protein accumulated in mosquito oocytes is a carboxypeptidase activated in embryos. Proceedings of the National Academies of Science. 88, 10821-10824.

Cho, K.H., Raikhel, A.S. 2001. Organization and developmental expression of the mosquito vitellogenin receptor gene. Insect Molecular Biology and Biochemistry. 10, 465-474.

Clements, A.N., 1992. The Biology of Mosquitoes. Chapman and Hall, London.

Clements, A.N., Boocock, M.R. 1984. Ovarian development in mosquitoes stages of growth and arrest and follicular resorption. Physiological Entomology. 9, 1-8.

Clifton, M.E., Noriega, F.G. 2011 Nutrient limitation results in juvenile hormonemediated resorption of previtellogenic ovarian follicles in mosquitoes. Journal of Insect Physiology. 57, 1274-1281.

Colless, D.H., Chellapah, W.T. 1960. Effects of body weight and size of bloodmeal upon egg production in Aedes aegypti (Linneaus) (Diptera: Culicidae) Annals of tropical medicine and parasitology. 54, 475-482.

Cooper, D.M., Thi, E.P., Chamberlain, C.M., Pio, F., Lowenberger, C. 2007. Aedes Dronc: a novel ecdysone-inducible caspase in the yellow fever mosquito, Aedes aegypti. Insect Molecular Biology. 16, 563-572.

Eichenlaub-Ritter, U., Peschke, M. 2002. Expression in in-vivo and in-vitro growing and maturing oocytes: focus on regulation of expression at the translational level. Human Reproduction Update. 8, 21-41. 
Fallon, A.M., Hagedorn, H.H., Wyatt, G.R., Laufer, H. 1974. Activation of vitellogenin synthesis in the mosquito Aedes aegypti by ecdysone, Journal of Insect Physiology. 20, 1815-1823.

Feinsod, F.M., Spielman, A., 1980. Nutritional-mediated juvenile hormone secretion in mosquitoes. Journal of Insect Physiology. 26, 113-117.

Flatt, T., Tu, M.P., Tatar, M. 2005. Hormonal pleiotropy and the juvenile hormone regulation of Drosophila development and life history. BioEssays 27, 9991010.

Fronstin, R.B., Hatle, J.D. 2008. A cumulative feeding threshold required for vitellogenesis can be obviated with juvenile hormone in lubber grasshoppers. Journal of Experimental Biology 211, 79-85.

Ferguson, E.M., Leese, H.J. 2006. A potential role for triglyceride as an energy source during bovine oocyte maturation and early embryo development. Molecular Reproduction and Development. 73, 1195-1201.

Gilchrist, R.B., Lane, M., Thompson, J.G. 2008. Oocyte-secreted factors: regulators of cumulus cell function and oocyte quality. Human Reproduction Update. 2, 159-177.

Gray, E.M., Bradley, T.J. 2003. Metabolic rate in female Culex tarsalis (Diptera: Culicidae): Age, size, activity, and feeding effects. Journal of Medical Entomology 40, 903-911.

Greenplate, J.T., Glaser, R.L., Hagedorn, H.H. 1985. The role of factors from the head in the regulation of egg development in the mosquito Aedes aegypti. Journal of Insect Physiology. 31, 323-329.

Gwadz, R.W., Spielman, A., 1973. Corpus allatum control of ovarian development in Aedes aegypti. Journal of Insect Physiology. 19, 14411448.

Hansen, I.A., Attardo, G.M., Park, J., Peng, Q., Raikhel, A.S. 2004. Target of rapamycin-mediated amino acid signaling in mosquito anautogeny. Proceedings of the National Academy of Sciences of the United States of America. 101, 10626-10631. 
Harshman, L.G., Zera, A. 2007. The cost of reproduction: the devil in the details. Trends in Ecology \& Evolution. 22, 80-86.

Highnam, K.C., Lusis, O., Hill, L. 1963a. The role of the corpora allata during oöcyte growth in the desert locust, Schistocerca gregaria Forsk. Journal of Insect Physiology. 9, 587-590.

Highnam, K.C. Lusis, O., Hill, L. 1963b. Factors affecting oöcyte resorption in the desert locust Schistocerca gregaria. Journal of Insect Physiology. 9, 827837.

Hopwood, J.A., Ahmed, A.M., Polwart, A., Williams, G.T., Hurd, H. 2001. Malariainduced apoptosis in mosquito ovaries: a mechanism to control vector egg production. Journal of Experimental Biology. 204, 2773-2780.

Homa, S.T., Racowsky, C., McCaughey, R.W. 1986. Lipid analysis of immature pig oocytes Journal of Reproduction and Fertility. 77, 425-434.

Jalil, M. 1974. Observations on the fecundity of Aedes triseriatus Diptera Culicidae. Entomologia Experimentalis Et Applicata. 17, 223-233.

Koller, C.N., Raikhel, A.S. 1991. Initiation of vitellogenin uptake and protein synthesis in the mosquito (Aedes aegypti) ovary in response to a blood meal. Journal of Insect Physiology. 37, 703-711.

Kokoza, V.A., Raikhel, A.S. 1997. Ovarian- and somatic-specific transcripts of the mosquito clathrin heavy chain gene generated by alternative 5 -exon splicing and polyadenylation. Journal of Biological Chemistry. 272, 11641170 .

Kokoza, V.A., Snigirevskaya, E.S., Raikhel, A.S. 1997. Mosquito clathrin heavy chain: analysis of protein structure and developmental expression in the ovary during vitellogenesis. Insect Molecular Biology. 6, 357-368.

Klowden, M.J. 1997. Endocrine aspects of mosquito reproduction. Archives of Insect Biochemistry and Physiology. 35, 491-512. 
Li, Y., Hernandez-Martinez, S., Unnithan, G.C., Feyereisen, R., Noriega, F.G. 2003. Activity of the corpora allata of adult female Aedes aegypti: Effects of mating and feeding. Insect Biochemistry and Molecular Biology. 33, 1307-1315.

Lea, A.O., Briegel, H., Lea, H.M. 1978. Arrest, resorption or maturation of oocytes in Aedes aegypti: dependence upon the quality of the blood meal and the interval between blood meals. Physiology Entomology. 3, 309316.

Leoni, G.G., Bebbere, D., Succu, S., Berlinguer, F., Mossa, F., Galioto, M., Bogli olo, L., Ledda, S., Naitana, S. 2007. Relations between relative mRNA abundance and developmental competence of ovine oocytes. Molecular Reproduction and Development. 74, 249-257.

Matorras, R., Ruiz, J.I., Mendoza, R., Ruiz, N., Sanjurjo, P., Rodriguez-Escudero, F.J. 1998 Fatty acid composition of fertilization-failed human oocytes. Human Reproduction. 13, 2227-2230.

Martin, D., Wang, S.F., Raikhel, A.S. 2001. The vitellogenin gene of the mosquito Aedes aegypti is a direct target of ecdysteroid receptor. Molecular and Cellular Endocrinology. 173, 75-86.

Mermillod, P.P., Dalbies-Tran, R.R., Uzbekova, S.S., Thelie, A.A., Traverso, J., Perreau, C.C., Monget, P.P. 2008. Factors affecting oocyte quality: Who is driving the follicle? Reproduction in Domestic Animals 43 (Suppl. 2), 393400 .

Mostowy, W.M., Foster, W.A. 2004. Antagonistic effects of energy status on meal size and egg-batch size of Aedes aegypti (Diptera: Culicidae). Journal of Vector Ecology. 29, 84-93.

Nagano, M., Katagiri, S., Takahashi, Y. 2006a. ATP content and maturational/developmental ability of bovine oocytes with various cytoplasmic morphologies. Zygote. 14, 299-304.

Nagano, M., Katagiri, S., Takahashi, Y. 2006b. Relationship between bovine oocyte morphology and in vitro developmental potential. Zygote. 14, 5361. 
Nayar, J.K., Sauerman, Jr. D.M. 1975. The effects of nutrition on survival and fecundity in Florida mosquitoes. Part 2: Utilization of a blood meal for survival. Journal of Medical Entomology. 12, 99-103.

Noriega, F.G., Wells, M. 1993. A comparison of three methods to isolate RNA from mosquitoes. Insect Molecular Biology. 2, 21-24.

Raikhel, A.S., Dhadialla, T.S., 1992. Accumulation of yolk proteins in insect oocytes. Annual Review Entomology. 37, 217-251.

Raikhel, A.S., Lea, A.O., 1983. Previtellogenic development and vitellogenin synthesis in the fat body of a mosquito: An ultrastructural and immunocytochemical study, Tissue and Cell. 15, 281-299.

Raikhel, A.S., Lea, A.O. 1985. Hormone mediated formation of the endocytotic complex in mosquitoes. General and Comparative Endocrinology. 57, 422-433.

Ramirez-Zacarias, J.L., Castro-Munozledo, F., Kuri-Harcuch, W. 1992. Quantitation of adipose conversion and triglycerides by staining intracytoplasmic lipids with oil red O. Histochemistry. 97, 493-497.

Roth, T.F., Porter, K.R. 1964. Yolk protein uptake in the oocyte of the mosquito Aedes aegypti. Cell Biology. 20, 313-332.

Sappington, T.W., Kokoza, V.A., Cho, W.L., Raikhel, A.S. 1996. Molecular characterization of the mosquito vitellogenin receptor reveals unexpected high homology to the Drosophila yolk protein receptor. Proceedings of the National Academies of Sciences. 93, 8934-8939.

Sarfati, M., Krasnov, B.R., Ghazaryan, L., Khokhlova, I.S., Fielden, L.J., Degen, A.A. 2005. Energy costs of blood digestion in a host-specific haematophagous parasite. Journal of Experimental Biology. 208, 2489-96.

Schlaeger, D.A., Fuchs, M.S. 1974. Localization of dopa-decarboxylase in adult Aedes aegypti females. Journal of Experimental Zoology. 187, 217-222. 
Seo, S.J., Cheon, H.M., Sun, J., Sappington, T.W., Raikhel, A.S. 2003. Tissueand stage- specific expression of two lipophorin receptor variants with seven and eight ligand- binding repeats in the adult mosquito. Journal of Biological Chemistry. 278, 41954-41962.

Spielman, A., Gwadz, R.W., Anderson, W.A., 1971. Ecdysone-initiated ovarian development in mosquitoes, Journal of Insect Physiology. 17, 1807-1814.

Sun, J., Hiraoka, T., Dittmer, N.T., Cho, K.H., Raikhel, A.S. 2000. Lipophorin as a yolk protein precursor in the mosquito, Aedes aegypti. Insect Biochemistry and Molecular Biology. 30, 1161-1171.

Sturmey, R.G., Leese, H.J., 2003. Energy metabolism in pig oocytes and early embryos. Reproduction. 126, 197-204.

Soller, M., Bownes, M., Kubli, E., 1999. Control of oocyte maturation in sexually mature Drosophila females. Developmental Biology. 208, 337-351.

Tadkowski, T.M., Jones, J.C. 1978. Endogenous synthesis of lipid yolk in mosquito oocytes. Experientia. 34, 627-627.

Thomson, T.C., Fitzpatrick, K.E., Johnson, J. 2010. Intrinsic and extrinsic mechanisms of oocyte loss. Molecular Human Reproduction. 16, 916-927.

Terashima, J., Takaki, K., Sakurai, S., Bownes, M., 2005. Nutritional status affects 20- hydroxyecdysone concentration and progression of oogenesis in Drosophila melanogaster. Journal of Endocrinology. 187, 69-79.

Tobe, S.S., Chapman, C.S., 1979. Effects of starvation and subsequent feeding on juvenile hormone synthesis and oocyte growth in Schistocerca americana gregaria. Journal of Insect Physiology 25, 701-708.

Troy, S., Anderson W.A., Spielman, A. 1975. Lipid content of maturing ovaries of Aedes aegypti mosquitoes. Comparative Biochemistry and Physiology. 50, 457-461.

Uchida, K., Moribayashi, A. 2002. Effects of physiological and unphysiological doses of 20-hydroxyecdysone on ovarian follicles in female Culex pipiens pallens (Diptera: Culicidae). Medical Entomology and Zoology. 53, 153161. 
Uchida, K., Ohmorib, D., Eshita, Y., Oda, T., Kato, Y., Tomizawa, K., Moribayashi, A., Fukunaga, A. 1998. Ovarian development induced in decapitated female Culex pipiens pallens mosquitoes by infusion of physiological quantities of 20-hydroxyecdysone together with amino acids, Journal of Insect Physiology. 44, 525-528.

Winckler, J., 1974. Vital staining of lysosomes and other cell organelles of the rat with neutral red. Progress in Histochemistry and Cytochemistry. 6, 1-91.

Weaver, R.J., Pratt, G.E. 1981. Effects of starvation and feeding upon corpus allatum activity and oocyte growth in adult female Periplaneta americana. Journal of Insect Physiology. 27, 75-83.

Woke, P.A., Ally, M.S., Rosenberger, C.R. Jr. 1956. The numbers of eggs developed related to the quantities of human blood ingested in Aedes aegypti. Annals of the Entomological Society of America. 49, 435-441.

Wrenzycki, C., Hermann, D., Lemme, E., Korsawe, K., Carnwath, J.W., Niemann, H. 1998. Transcriptional level of developmentally important genes in bovine preimplantation embryos generated in vitro, Theriogenology. 49, 191.

Zera, A.J., Zhao, Z.W. 2004. Effect of a juvenile hormone analogue on lipid metabolism in a wing-polymorphic cricket: Implications for the endocrinebiochemical bases of life-history trade-offs. Physiological Biochemical Zoology. 77, 255-266.

Zhou, G., Pennington, J.E., Wells, M.A., 2004a. Utilization of pre-existing energy stores of female Aedes aegypti mosquitoes during the first gonotrophic cycle. Insect Biochemistry and Molecular Biology. 34, 919-25.

Zhou, G. L., Flowers, M., Friedrich, K., Horton, J., Pennington, J. Wells, M. A. 2004b. Metabolic fate of [C-14]-labeled meal protein amino acids in Aedes aegypti mosquitoes. Journal of Insect Physiology. 50, 337-349.

Ziegler, R., Ibrahim, M.M. 2001. Formation of lipid reserves in fat body and eggs of the yellow fever mosquito, Aedes aegypti. Journal Insect Physiology. $47,623-27$. 
Ziegler, R., Van Antwerpen, R. 2006.Lipid uptake by insect oocytes. Insect Biochemistry and Molecular Biology. 36, 264-72

Ziegler, R. 1997. Lipid synthesis by ovaries and fat body of Aedes aegypti (Diptera: Culicidae) European Journal of Entomology. 94, 385-91. 


\section{CHAPTER 5: Male Aedes aegypti use JH III transferred during copulation to}

influence previtellogenic ovary physiology and affect the reproductive output of female mosquitoes

\subsection{Abstract}

The effect of male accessory gland substances on female reproductive physiology has been described as "activating" egg development. However, no mechanism has been described that can explain how male mosquitoes are able to influence egg development in female mosquitoes. To investigate how male mosquitoes are able to influence ovarian physiology and reproductive output we explored three main questions: 1) Does mating and male accessory gland substances affect ovarian physiology, and alter markers of oocyte quality during the previtellogenic resting stage? 2) Does the male accessory gland contain $\mathrm{JH}$ and is $\mathrm{JH}$ transferred to the female during copulation? 3) and finally, does the nutritional history of the male effect reproductive output in the female? By answering these questions it is clear that male mosquitoes are able to alter the female's resource allocation priorities towards reproduction by transferring $\mathrm{JH}$ during copulation; reducing the rate of previtellogenic resorption, increase the amount of stored ovarian lipids and improving a follicle's likelihood of development after a blood meal. In addition, males maintained under better nutritional conditions exert a stronger effect and therefore are likely to realize higher fecundities than other males. Together these results illustrate one mechanism behind the activating effect of mating described 


\subsection{Introduction}

Mating causes profound changes to the female mosquito's physiology and behavior. Besides inducing refractoriness to further mating (Craig, 1967; Spielman et al., 1967), altering host-seeking/biting behavior (Lavoipierre, 1958; Klowden and Lea, 1979), as well as oviposition behavior (Hiss and Fuchs, 1972; Yeh and Klowden, 1990) mating also effects egg development physiology. Work by Klowden and Chambers (1991) found that the proportion of starved females that developed eggs after a small blood meal increases when the females where mated and this effect was a result of substances produced by the male accessory gland. In the absence of accessory gland substances, the activation of egg development after a small blood meal could be replicated through topical juvenile hormone (JH) application. Similar work by Uchida et al. (2003) reported that haemocoel infusion with an amino acid mixture was more likely to induce oogenesis in mated Anopheles stephensi females than unmated females. In the autogenic mosquito Aedes taeniorhynchus, mating was shown to induce autogeny when mated females were more likely to develop eggs without a blood meal (O'meara and Evans, 1976; O'meara and Evans, 1977). In all of these prior works, the effect of accessory gland substances has been described as "activating" or "enhancing" egg development and shows that mating seems to alter resource allocation priorities towards reproductive physiology (The relationship between accessory gland substances and egg development has been thoroughly reviewed in Gillott, 2003). 
Similarly to Aedes aegypti, female Drosophila melanogaster also show enhanced reproductive physiology after mating. Male D. melanogaster accessory gland proteins such as ovulin (Acp26a) and sex-peptide (Acp70a) cause increased rates of oogenesis, ovulation and egg-laying (reviewed in Gillott, 2003 and in Wolfner, 1997, 2002 and 2009). Bioinformatic characterizations of male accessory gland substances in $A$. aegypti has shown that, also similarly to $D$. melanogaster, the male accessory gland contribution includes a variety of proteins with the potential to exert effects on female physiology and behavior (Sirot et al., 2008; Sirot et al., 2011). However, work by Li et al. (2003) clearly demonstrated where the similarities end when mating failed to increase $\mathrm{JH}$ synthesis rates in $A$. aegypti despite clearly doing so in $D$. melanogaster (Moshitsky et al., 1996). Together these results suggest that although the male $A$. aegypti accessory gland substances possess the ability to influence egg development, increasing rates of $\mathrm{JH}$ synthesis to achieve this end does not seem to be one of its functions. To our knowledge, no mechanism has been described in mosquitoes that can fully explain how male reproductive contributions activate or enhance reproduction, alter fecundity, or increase the proportion of females that develop eggs. Despite the absence of a clear effect of mating on endogenous $\mathrm{JH}$ synthesis rates, the repeatedly described pattern of activated ovarian physiology after mating in mosquitoes strongly suggests that the male effect on ovarian physiology is allohormonal in nature (Gillott, 2003).

Previous work in our laboratory that explored the effect of nutrition and $\mathrm{JH}$ during the previtellogenic resting stage in $A$. aegypti determined that critical 
decisions about reproductive output are made during the previtellogenic stage. Most importantly, it was observed that the quality of oocytes during the previtellogenic resting stage determines their likelihood of successfully completing oogenesis after a blood meal. Oocytes from poorly nourished mosquitoes were more likely to resorb before and after a blood meal and, conversely, oocytes from mosquitoes that are well-nourished or treated with $\mathrm{JH}$ analogue are of a higher quality and have a higher likelihood of completing oogenesis. Therefore, reproductive output and the developmental fate of a follicle after a blood meal are highly dependent on nutritional conditions during the previtellogenic resting stage and this effect is mediated through $\mathrm{JH}$ signaling (Clifton and Noriega, 2012). The observation that the previtellogenic ovarian follicle is dependent on $\mathrm{JH}$ signaling to determine its developmental fate, when taken together with previous works showing the "activating" and "enhancing" effects of mating on egg development suggests that male accessory gland substances may be acting during the previtellogenic stage to alter ovarian physiology thereby affecting reproductive output.

More specifically, we hypothesized that the male contribution would act on female physiology similarly to topical JH III application by improving previtellogenic oocyte quality (and therefore the likelihood of developing after a blood meal), reducing resorption during the previtellogenic resting stage, and ultimately causing an increase in reproductive output (Clifton and Noriega, 2012). We also hypothesized that similarly to some lepidopteran species, male $A$. aegypti mosquitoes likely exert this reproductive effect by transferring $\mathrm{JH}$ III 
contained in the accessory gland to their female partners during copulation (Park et al., 1998). The presence of $\mathrm{JH}$ III in the accessory gland has been reported previously by Borovsky et al. (1994). The hypothesis that JH III is transferred to females during copulation provides a mechanism that can easily explain, at least in part, how mating is able to enhance the previtellogenic quality of eggs, increase their likelihood of developing after a blood meal, and generate the "activating" or "enhancing" effect described in previous works. Together these hypotheses begin to explain the mechanism by which male mosquitoes are able to alter the resource allocation balance within the female and cause the enhancement of reproduction reported in Chambers and Klowden (1991) and ubiquitous among insects after mating (Gillott, 2003). Finally, these hypotheses also suggest that the nutritional status of the male mosquito may determine the magnitude of the effect seen on female physiology.

To investigate how male mosquitoes are able to influence ovarian physiology and reproductive output in female mosquitoes we explored three main questions: 1) Does mating and male accessory gland substances affect ovarian physiology, and alter markers of oocyte quality during the previtellogenic resting stage? 2) Does the male accessory gland contain $\mathrm{JH}$ III and is $\mathrm{JH}$ III transferred to the female during copulation? 3) and finally, does the nutritional history of the male effect reproductive output in the female? By answering these questions it is clear that male mosquitoes are able to alter the female's resource allocation priorities towards reproduction by transferring $\mathrm{JH}$ III during copulation and effecting the rate of previtellogenic resorption as well as a follicle's likelihood of 
development after a blood meal. In addition, it is clear that males maintained under better nutritional conditions exert a stronger effect and therefore are likely to realize higher fecundities than other males.

\subsection{Methods}

\subsubsection{Insects and mating}

A colony of $A$. aegypti of the Rockefeller strain was maintained at $28^{\circ} \mathrm{C}$ with $80 \%$ relative humidity under a $16 \mathrm{~h}$ day- $8 \mathrm{~h}$ night regime. 24 hours after pupation mosquitoes were sorted by size into female only (generally larger pupae) and male only populations (smaller pupae). Populations of mosquitoes of both sexes were divided again and offered a cotton pad soaked with either $3 \%$ sucrose solution or $20 \%$ sucrose solution. This procedure yielded completely virgin male and virgin female mosquitoes maintained on two different nutritional regimes for all experiments.

To investigate the effects of mating and nutrition on previtellogenic resorption, virgin female mosquitoes were maintained alone with a cotton pad soaked in either $3 \%$ sucrose solution or $20 \%$ sucrose solution (unmated treatments) or they were mixed with virgin male mosquitoes 3 days after emergence in a $1: 1$ ratio and maintained on $3 \%$ sucrose or $20 \%$ sucrose (mated treatments). This procedure yielded mated and unmated female mosquito populations fed either $3 \%$ sucrose or $20 \%$ sucrose. At 6-7 days after emergence,

mosquitoes from at least three independent biological replicates were dissected and resorption and lipid content of ovaries were determined. 
To determine the effect of male nutritional history alone on female previtellogenic resorption, 3 day old virgin females maintained on $3 \%$ sucrose were mated in a 1:1 ratio with males maintained on either $3 \%$ sucrose or with males maintained on $20 \%$ sucrose. All female mosquitoes were maintained on $3 \%$ sucrose after mixing with male mosquitoes. At 6-7 days after emergence (34 days after introduction to males) female mosquitoes were anesthetized and the proportion of resorbing follicles as well as the neutral lipid content of ovaries was determined as previously described.

To determine total egg output, female mosquitoes mated with males that were maintained on either $3 \%$ sucrose or $20 \%$ sucrose were blood fed at $6-7$ days after emergence, separated into groups of 5 fully engorged individuals in quintuplicate and allowed to lay eggs on paper. The total number of eggs laid was counted by dissecting microscope and divided by the number of females to determine average number of eggs per female. This entire experiment was conducted for three independent biological replicates.

\subsubsection{Measurements of follicular resorption and ovarian lipids}

The rate of follicular resorption in ovaries of female mosquitoes was determined as described in chapter 3.3.2 (p. 40). Ovarian lipids were measured as described in chapter 4.3 .3 (p. 77).

\subsubsection{Accessory gland injections}

To investigate the role of accessory gland substances separate from sperm related effects or other interference from the act of mating, virgin female mosquitoes were injected with crude male accessory gland homogenates. The 
accessory glands from 20 anesthetized male mosquitoes were dissected, rinsed in PBS and homogenized via sonication in 10ul of PBS. The homogenate was centrifuged briefly and $0.414 \mathrm{uL}$ ( 0.8 male accessory gland equivalent) was injected using a Drummond Nanoject II microinjector. Resorption and lipid content of ovaries was determined in triplicate 3-4 days after injection as previously described.

\subsubsection{Measurements of $\mathrm{JH}$ titers in male accessory glands, and female bursa copulatrix/ spermathecae complexes}

Borovsky et al., (1994) previously described the synthesis of JH III in the accessory gland of male $A$. aegypti. To investigate the possibility that male mosquitoes possess and pass on $\mathrm{JH}$ III contained in their accessory glands to their female partners during copulation we mixed 20 female mosquitoes maintained on $3 \%$ sucrose with 20 males maintained on $20 \%$ sucrose in triplicate. Mosquitoes were left undisturbed for 2 hours to allow mating to occur. Female mosquitoes were anesthetized by chilling and the 9th tergite attached to the spermathecae and bursa copulatrix was pulled away from the abdomen, rinsed in PBS and assayed for JH III content using an HPLC-FD quantification method. The accessory glands from 10 virgin males fed $3 \%$ and $20 \%$ sucrose as well as mated males fed $20 \%$ sucrose were also dissected in triplicate and assayed for JH III content.

To quantify $\mathrm{JH}$ III in biological samples the following method (currently submitted to PLos one, Rivera-Perez, 2012) was used. All tissues were incubated for $4 \mathrm{~h}$ in the dark at $32{ }^{\circ} \mathrm{C}$ under continuous gentle agitation in M-199 
tissue culture medium (Lavallette, NJ, USA). 2\% Ficoll, 25 mM HEPES (pH 6.5) and methionine $(50 \mu \mathrm{M})$ was added to the tissue culture medium. After incubation $150 \mu \mathrm{l}$ of $100 \mathrm{mM}$ sodium sulfide was added and the epoxide ring of $\mathrm{JH}$ III was opened by heating the biological extracts for $30 \mathrm{~min}$ at $55^{\circ} \mathrm{C}$. To extract $\mathrm{JH}$ III, $500 \mu \mathrm{l}$ of hexane was added to samples. The vials were vortexed and sonicated for $5 \mathrm{~min}$ and centrifuged at $2000 \mathrm{~g}$ for $5 \mathrm{~min}$ at $4{ }^{\circ} \mathrm{C}$. The organic phases (upper layer) were removed and transferred to new vials. The extracted organic phases $(\sim 500 \mu \mathrm{l})$ were filtered with Nalgene 4-mm syringe filters $(0.2 \mu \mathrm{m}$ nylon membrane, \#176) and dried under a $\mathrm{N}_{2}$ atmosphere. Samples were reconstituted with $25 \mu \mathrm{l}$ of acetonitrile and $25 \mu \mathrm{l}$ of $1 \mathrm{mM}$ DBD-COCl (fluorescent tag) were added. Labeling mixtures were incubated at room temperature for 40 min and reactions were terminated by adding $50 \mu \mathrm{l}$ of acetonitrile. Aliquots of the reactions were analyzed by HPLC-FD.

A Dionex Summit System (Dionex, Sunnyvale, CA) equipped with a 680 HPLC pump, a TCC 100 column oven, a UV 170U detector and an UltiMate 3000 fluorescence detector connected in series was used in conjunction with Chromeleon software version 6.8 SR10 to perform HPLC-FD. The separation of tagged compounds was performed on an analytical column Acclaim 120 C18 $(250 \times 2.1 \mathrm{~mm}$ ID, particle size $5 \mu \mathrm{m})$ (Dionex), using isocratic elution from 0 to $20 \mathrm{~min}$ (acetonitrile/water, 1 to $1 \mathrm{v} / \mathrm{v}$ ), followed by a linear gradient from 20 to 50 $\min ($ acetonitrile-water (50 to 95\%, v/v) and another isocratic elution from $50 \mathrm{~min}$ (acetonitrile, 95\%). Flow rate was $0.2 \mathrm{ml} / \mathrm{min}$ and column temperature was $25^{\circ} \mathrm{C}$. 
The eluate was monitored with UV (214 nm) and fluorescence detection with the wavelengths for $\lambda_{\text {ex. }} 450 \mathrm{~nm}$ excitation and $\lambda_{\mathrm{em} .} .560 \mathrm{~nm}$ for emission.

\subsection{Results}

\subsubsection{Mating reduces the rate of previtellogenic resorption}

When female mosquitoes fed $3 \%$ sucrose were mated at 3 days old and assayed 3-4 days later, the rate of resorption was reduced from $7.99 \%$ follicles per female to $4.91 \%$ of follicles per female (Figure 18; $p=.0004$; unpaired t-test). When this experiment was repeated with female mosquitoes maintained on $20 \%$ sucrose, the rate of resorption decreased from $5.69 \%$ of follicles in unmated mosquitoes to $3.27 \%$ of follicles in mated mosquitoes (Figure 18; $p=.003$; unpaired t-test).

\subsubsection{Mating increases previtellogenic lipid content of follicles}

Previous work determined that follicular resorption is inversely proportional to lipid content such that ovaries with higher lipids (from females maintained on higher sucrose concentrations) will have lower rates of resorption. The neutral lipid content of previtellogenic ovarian follicles is also reflective of previtellogenic nutritional status, is mediated by $\mathrm{JH}$ and is a marker of a follicle's likelihood of developing after a blood meal (Clifton and Noriega, 2012). The neutral lipid content of ovaries increased when mosquitoes were mated. Although not statistically significant, in female mosquitoes fed $3 \%$ sucrose solution and mated, the lipid content of the ovaries increased $26 \%$ from 158.60 pmol./female to 214.70 pmol./female (Figure 19; $p=.068$; Mann-Whitney U-test). When the same

experiment was repeated with females fed $20 \%$ sucrose, the neutral lipid content 
of the ovaries increased 2-fold from 229.3 pmol./female to 567.9 pmol./female in mated mosquitoes (Figure 19; $p \leq .0001$; Mann-Whitney U-test).

\subsubsection{Male nutritional history affects previtellogenic ovary lipid content, the previtellogenic rate of resorption as well as reproductive output}

Offering male mosquitoes $20 \%$ sucrose increases the magnitude of the mating effect when compared to males maintained on $3 \%$ sucrose. When female mosquitoes maintained on $3 \%$ sucrose were mated to males maintained on $3 \%$ sucrose and assayed 4 days later, the rate of resorption was $6.66 \%$ (Figure 20A). When this experiment was repeated with males maintained on $20 \%$ sucrose, the rate of resorption decreased to $3.96 \%$ (Figure 20B; $p=.0018$; Unpaired t-test). Concomitant with an decrease in the rate of resorption, the neutral lipid content of ovaries increased 3-fold when female mosquitoes were mated with $20 \%$ males (Figure 20B; 331 pmol./female vs. 889.7 pmol./female; $\mathrm{p}=.042$; Mann-Whitney U-test).

Male nutritional history also affected the reproductive output of female mosquitoes. When female mosquitoes where mated with males maintained on $3 \%$ sucrose, blood fed and allowed to lay eggs, the reproductive output was 120.0 eggs/female. When females were mated to males maintained on $20 \%$ sucrose, reproductive output rose to 129.2 eggs/female; an increase of over $9 \%$ (Figure 21; $p=.030$; Unpaired t-test). To discount the possibility that male nutritional history alters allocations to individual eggs we also investigated the lipid content of eggs resulting from each treatment. Eggs from females mated with $3 \%$ sucrose males or $20 \%$ sucrose males did not differ significantly in 
neutral lipid content (342.9 pmol./egg vs. 380.2 pmol./egg; $p=.24$; Mann-Whitney U-test).

\subsubsection{Male accessory gland substances increase lipid content and reduce resorption}

To demonstrate that the effect of mating on female previtellogenic ovary physiology is caused by substances within the male accessory gland and not the result of pheromones or the physical act of mating, we injected homogenates of male accessory glands into 4 day old females maintained on $3 \%$ sucrose. Injecting accessory gland homogenates lowered the rate of resorption from $5.93 \%$ in PBS injected mosquitoes to $3.33 \%$ in mosquitoes injected with accessory gland homogenates (Figure 22A; $\mathrm{p}=.0063$; Unpaired t-test). Injecting male accessory gland homogenates also caused the lipid content of ovaries to increase $34 \%$ from $253.6 \mathrm{pmol}$. of triglyceride/pair of ovaries to $388.5 \mathrm{pmol}$. of triglyceride/pair of ovaries (Figure 22B; $p=.05$; Mann-Whitney U-test).

\subsubsection{Male mosquitoes transfer JH III to the female during copulation}

In addition to determining the effect of mating on ovary physiology and female fecundity, we also investigated the effect of sugar feeding on $\mathrm{JH}$ accumulation within the accessory gland. Males fed $3 \%$ sucrose accumulated $5.3 \mathrm{fmol}$. of $\mathrm{JH}$ after 5 days of sugar feeding. When males were maintained on $20 \%$ sucrose the amount of $\mathrm{JH}$ within the accessory gland increased to $8.4 \mathrm{fmol}$. of $\mathrm{JH}$ during the same time. After 2 hours of mating, the amount of $\mathrm{JH}$ in the accessory gland of males fed $20 \%$ sucrose decreased to $4.3 \mathrm{fmol}$. of $\mathrm{JH} /$ accessory gland (Figure 23; $\mathrm{p}=.05$; Mann-Whitney U-test). 
To determine if the decrease of $\mathrm{JH}$ within the accessory gland after mating was being transferred during copulation we dissected bursa copulatrixspermathecae (BC-S) complexes from virgin females as well as from female mosquitoes immediately after copulation with males maintained on $20 \%$ sucrose. The JH III content of the BC-S increased by 36.15 fmol./BC-S (from 8.58 fmol./BC-S in unmated females to 44.73 fmol./BC-S in mated females; Figure 24; $p=.05$; Mann-Whitney U-test) in females after mating suggesting the transfer of $\mathrm{JH}$ III from male to female during copulation.

\subsection{Discussion}

\subsubsection{JH pleiotropy and resource allocation}

The allocation of incoming resources has often been described using a $\mathrm{Y}$ model of resource allocation whereby resources entering at the stem of the "Y" are allocated towards either reproductive physiology on one branch and/or somatic physiology on the other (De Jong and Van Noordwijk, 1992; Tatar and Carey, 1995; Harshman and Zera, 2007; Boggs, 2009; Chapter 2 (p. 25)). Under this model, excessive allocations toward somatic physiology or reductions in incoming nutrition often result in reductions to reproductive output (Partridge et al., 1987; Tatar and Carey, 1995). Alternatively, experimental manipulations that reduce reproductive output can result in increased longevity consistent with the Y-model (Lamb, 1964; Djawdan, 1996; Tater and Carey, 1995). Despite the shortcomings of current resource allocation models (For example, Flatt and Kawecki (2007) found that fecundity may not always trade-off with increased lifespan) reproductive output in insects has been shown in numerous instances 
to trade-off against somatic physiology such as: flight and flight physiology (Olivieras et al., 2006; Zera and Brink, 2000; Socha and Sula, 2008 ), adult phenotype (Moczek, 1998; Moczek and Nijhout, 2004), and in response to nutritional status (Boggs and Ross, 1993; Ohgushi, 1996; Osawa, 2005). Somatic and reproductive trade-offs have been shown many times to also be mediated through $\mathrm{JH}$ signaling. $\mathrm{JH}$ performs a central role as a mediator of lifehistory strategy and fitness trade-offs in many insects (reviewed Flatt and Tatar, 2005) and therefore fits comfortably within the Y-model as the hormonal mediator of the reproductive/soma balance (Harshman and Zera, 2007). The idea that JH "hormonal pleiotropy" (Flatt and Tatar, 2005) plays a key allocation role within the conceptual framework of the $\mathrm{Y}$-model has been explicitly tested and the results have demonstrated that the biochemical and molecular mechanisms behind allocations to somatic or reproductive physiology can be mediated through $\mathrm{JH}$ action (Reviewed in Harshman and Zera, 2007). Juvenile hormone's classically defined role as an insect gonadotropin clearly illustrate this hormone's ability to alter the resource allocation balance towards reproductive physiology in a variety of insects (Hartfelder, 2000). Therefore, interpreting the functions of $\mathrm{JH}$ within the context of the Y-model could represent a powerful conceptual tool for investigating and understanding reproductive trade-offs as well as the regulation of reproductive output under properly controlled conditions. 


\subsubsection{Male accessory gland substances alter the resource allocation within the female insect}

Male insects frequently pass along substances to females during copulation which possess the ability to significantly alter female physiology, behavior and fecundity (Reviewed in Chen, 1984; Vahed, 1998; Gillott, 2003). By viewing male contributions within the context of a De Jong and Van Noordwijk's (1992) Y-model of resource allocation and Flatt and Tatar's descriptions of JH "hormonal pleiotropy", it is clear that in many cases, the end result of mating also manifests as an alteration to the internal resource allocation priorities within the female towards reproductive physiology (Merle, 1968; Klowden and Chambers, 1991; Reviewed in Gillott, 2003). In some examples, if the male contribution to reproduction involves nuptial (i.e., nutritional) gifts, female fecundity can be enhanced (Thornhill, 1976; Gwynne, 1984; Boggs, 1990; Reviewed in Gwynne, 2008), simultaneous with improvements in survivorship or longevity (Boggs, 1990; Fox, 1993). When the nuptial gift is viewed under the context of the Ymodel its effects frequently appear as an alteration to the resource balance within a female by providing additional nutritional resources at the stem of the "Y" presumably benefiting both somatic and reproductive physiology at the female insects discretion. To explore the possibility that male $A$. aegypti accessory gland substances may simultaneously benefit reproductive physiology as well as survivorship through a nuptial, trophic or nutritional effect, we compared survivorship in starved unmated female mosquitoes to survivorship in starved female mosquitoes mated with males maintained on both $3 \%$ sucrose and $20 \%$ 
sucrose. In none of these experiments were we able to definitively observe any benefits to survivorship in any of the starved and mated mosquitoes (results not shown). Klowden and Chambers, (1991) also conducted a similar survivorship experiments and reported that mating was detrimental to survivorship during starvation. However, results by Lavoipierre (1958) and Helinski and Harrington (2011) both demonstrated that mating confers an increase in longevity in continuously fed mosquitoes. While we did not explore the effect of mating on longevity (only survivorship under starvation conditions), the totality of these observations suggest that the male accessory gland substance, while seemingly neutral or even detrimental during starvation, confers benefits to fed females. Since the male accessory gland contribution contains a variety of proteins with unknown physiological functions (Sirot et al., 2008), it is likely that at least some of these products may produce the discrepancy described here.

Many insects do not pass along explicitly nutritional gifts, but instead pass substances produced by the male accessory gland with the capacity to alter female reproductive physiology including proteins (Reviewed in Wolfner, 1997; Dottorini, 2007; Sirot et al., 2008; Sirot et al., 2011), and hormones such as JH (Park et al.,1998) and ecdysteroids (Pondeville et al., 2008). Similarly to insects that pass nuptial gifts, male contributions that are not specifically nutritional (i.e. hormones, allohormones, or other physiologically active substances) can also increase fecundity and benefit male fitness (Reviewed in Wolfner, 1997; Shu et al., 1998). To investigate the activity of male accessory gland substances in the absence of any mating, pheromone or physical stimuli, we injected accessory 
gland homogenates into the female mosquito. With this technique we were able to demonstrate an ovarian phenotype that was remarkably similar to the phenotype obtained after mating. Injected male accessory substances were able to lower resorption (Figure 22A) and increase the neutral lipid content of the ovaries (Figure 22B) similar to mating (Figures 18 and 19) or $\mathrm{JH}$ analogue application (Clifton and Noriega, 2012). This result suggests that the effects on reproductive physiology observed in female mosquitoes after mating are most likely due to the substances contained within the accessory gland and are not exclusively the result of physical, pheromonal, or other stimuli.

By determining that the activating effect of mating on reproduction in $A$. aegypti is due to substances contained within the accessory gland and that these substances exert effects on ovarian physiology and fecundity that are similar to $\mathrm{JH}$ III application we reasoned that a hormonal factor was likely responsible for these observations. In agreement with results obtained by Borovsky et al., (1994) we also found significant quantities of $\mathrm{JH} \mathrm{III}$ in the accessory gland of male mosquitoes. When we quantified $\mathrm{JH}$ III in the bursa copulatrix/spermathecae complex immediately after mating we found a 5 -fold increase in the quantity of $\mathrm{JH}$ III as compared to unmated controls suggesting that the male mosquito is able to alter $\mathrm{JH}$ titers within the female by donating $\mathrm{JH}$ III stored (Figure 23) in the accessory gland thereby increasing stored ovarian lipids (Figures 19), reducing resorption (Figures 18) and increasing fecundity (Figure 21). The transfer of JH III during copulation can explains how mating causes the shift towards reproductive physiology often described in the literature and demonstrates at 
least one mechanism by which male mosquitoes attempt to alter the resource allocation balance within the female. However, the functions of many of the male A. aegypti accessory gland proteins have yet to be identified so it is unlikely that $\mathrm{JH} \mathrm{III} \mathrm{is} \mathrm{the} \mathrm{only} \mathrm{physiologically} \mathrm{active} \mathrm{component} \mathrm{of} \mathrm{the} \mathrm{male} \mathrm{accessory} \mathrm{gland.}$

In $A$. aegypti, JH has been shown in a variety of conditions to alter the soma/reproductive balance. Hernandez-Martinez et al., (2007) demonstrated that removing the head of the female immediately after emergence eliminates $\mathrm{JH}$ synthesis, eliminates reproductive maturation and extends survivorship over mosquitoes who were permitted to mature ovaries before decapitation. Earlier work by Caroci et al., (2004) showed that starved larvae emerge as smaller adults, have lower $\mathrm{JH}$ III synthesis rates after emergence and do not mature their eggs completely until a blood meal is obtained or $\mathrm{JH}$ III is applied topically. Klowden and Chambers (1991) were able to show the stimulatory effects of JH application on egg development consistent with a gonadotropic role for $\mathrm{JH}$. Each of these works demonstrate that, at least during the previtellogenic stage, JH III mediates reproductive allocations consistent with models of resource allocation in $A$. aegypti.

More recent work in our lab was able to demonstrates how JH III-induced reproductive allocations during the previtellogenic stage have lasting consequences for reproductive output after a blood meal. More specifically, we determined final reproductive output is related to the neutral lipid and mRNA content of the previtellogenic ovary and that these markers of a follicle's likelihood of completing oogenesis (i.e. quality) are dependent on nutrition and 
$\mathrm{JH}$ III. We also demonstrated that the reproductive output of the mosquito after a blood meal could be increased by $\mathrm{JH}$ III application or improving nutrition during the previtellogenic resting stage (Clifton and Noriega, 2011; Clifton and Noriega, 2012). When we mated mosquitoes in this study, the lipid content of ovaries increased and the rate of resorption decreased, consistent with a shift in resource allocation priorities towards reproductive physiology (Figures 18 and 19) and surprisingly similar to the effects $\mathrm{JH}$ III or methoprene application we previously described. When we repeated this experiment after improving the previtellogenic nutrition of female mosquitoes, the effect of mating was magnified (Figures 18 and 19). We believe the reduction in follicular resorption and increase in stored neutral lipids after mating is the same activating or enhancing effect described by Klowden and Chambers (1991) and Uchida et al., (2003). Therefore, the simplest mechanism for a male mosquito to influence the female's resource allocation balance, reproductive output and his own fitness is likely through the manipulation of $\mathrm{JH}$ titers during the previtellogenic resting stage. Together these results show that male mosquitoes are able to improve both the likelihood of a follicle developing and their own fitness by altering aspects of ovarian physiology that are intricately connected to reproductive output.

\subsubsection{The male mosquitoes nutritional history alters the magnitude of the effect on female reproductive physiology}

The Y-model and other models like it have all attempted to describe the relationship between resource acquisition, longevity and reproduction in terms of female reproductive output. However, male mosquitoes must also acquire 
resources and contribute in some way towards reproduction. In male A. aegypti the contribution towards reproduction is not likely to be insignificant. It has been reported many times that the accessory glands of male mosquitoes are depleted after copulation with 4-8 females which suggests that substances made within the accessory gland are costly to produce and are not unlimited (Judson, 1967; Foster and Lea, 1975; Helinski and Harrington, 2011). Work that altered larval nutrition and explored the effect of a small male phenotype on female found that small males are depleted faster and are unable to inseminate as many females and any beneficial effects of mating on female longevity were reduced in these males (Helinski and Harrington, 2011). Fernandez and Klowden (1995) also obtained results that suggest the nutritional history of the male may be an important factor when determining the extent of the accessory gland effect on female physiology and were able to demonstrate that starved males had less protein in their accessory glands, transferred less protein to females during copulation and exerted a smaller influence on female host-seeking behavior. These results begin to suggest that the resource allocation balance within the male mosquito may play an important role in determining his reproductive contribution. Therefore we hypothesized that improving the quality of adult male nutrition may increase the effects of his reproductive contribution and this effect would manifest in the aforementioned markers of female ovary quality, physiology and most importantly, fecundity. When we improved the nutritional history of the male mosquito by offering $20 \%$ sucrose we were able to increase the magnitude of the effects on female reproductive physiology. Males 
maintained on $20 \%$ sucrose were able to reduce the rate of resorption (Figure 20A) and dramatically increase the lipid content of the ovary (Figure 20B). When females mated to males fed $20 \%$ sucrose were blood fed, they were able to produce 9 more eggs than females mated to males fed $3 \%$ sucrose during the first gonotrophic cycle (120.0 vs. 129.2). In addition, the JH content of accessory glands from males fed $20 \%$ sucrose was $37 \%$ higher than males fed $3 \%$ sucrose (Figure 23). This result contrasts with work by Helinski and Harrington (2011) who were unable to affect female reproductive output through alterations in male larval nutrition. However, in our experimental design we manipulated the sugar concentration offered to adult males raised under the same larval conditions rather than the larval conditions themselves.

While reproductive output as a function of male nutritional history and accessory gland investment has been rarely studied, some work has shown that $\mathrm{JH}$ transferred by the male can influence reproductive output. In the moth Heliothis virescens, Shu et al. (1998) and Park et al. (1998) showed that male accessory glands contain $\mathrm{JH}$ as well as induce the endogenous synthesis of $\mathrm{JH}$ in female insects. The increased titers of $\mathrm{JH}$ after mating correlated tightly with reproductive output suggesting that male insects are able to exert influence over fecundity through $\mathrm{JH}$ transfer. However, no effect of male nutritional history on female reproductive output in mosquitoes has been described before. Together these results demonstrate that male mosquitoes are able to influence their own fitness and that male mosquitoes of a better nutritional state are able to realize 
higher fecundities than male mosquitoes maintained on $3 \%$ sucrose and that this effect is a result of $\mathrm{JH}$ transfer.

\subsubsection{Conclusions}

In this study we examined the male mosquito accessory gland contribution and determined that mating is able to alter markers of previtellogenic ovary quality such as the neutral lipid content of the ovary and the rate of follicular resorption. We also determined that $\mathrm{JH}$ III contained within the accessory gland is likely responsible for the alterations to ovarian physiology we observed. We were able to show that improving the quality of nutrition offered to male mosquitoes was able to magnify the effect on female reproductive physiology. When these results are taken together they illustrate that by manipulating $\mathrm{JH}$ III titers during the previtellogenic resting stage, the male mosquito is able to increase his own fecundity by improving a follicle's likelihood of developing after the female mosquito feeds on blood. These results add an important facet to our understanding of the determinants of reproductive output in this disease vector.

\subsection{Acknowledgments}

We thank Dr. Marcela Nouzova and Stefano Correa for constant rearing and separating of mosquitoes as well as Dr. Marcela Nouzova, Dr. Crisalejandra Rivera-Perez and Mario Perez for critical reading of the manuscript. This work was supported by NIH Grant No. Al 45545 to F.G.N. 


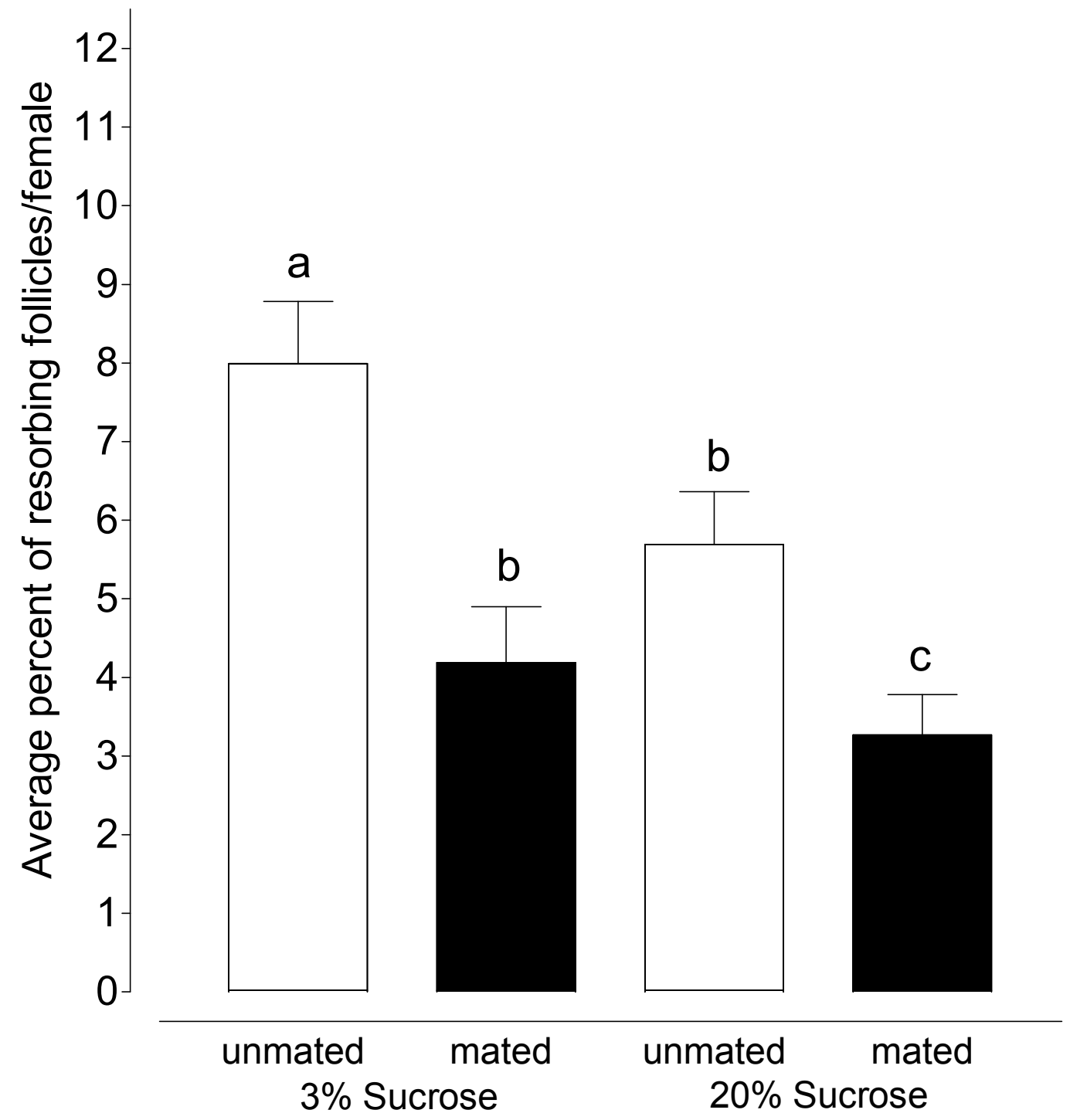

Figure 18: Mating decreases the average rate of resorption in females maintained on $3 \%$ sucrose and $20 \%$ sucrose (Mean \pm SEM; unpaired t-test; Treatments with different letters denote statistical significance at $p \leq .01)$. 


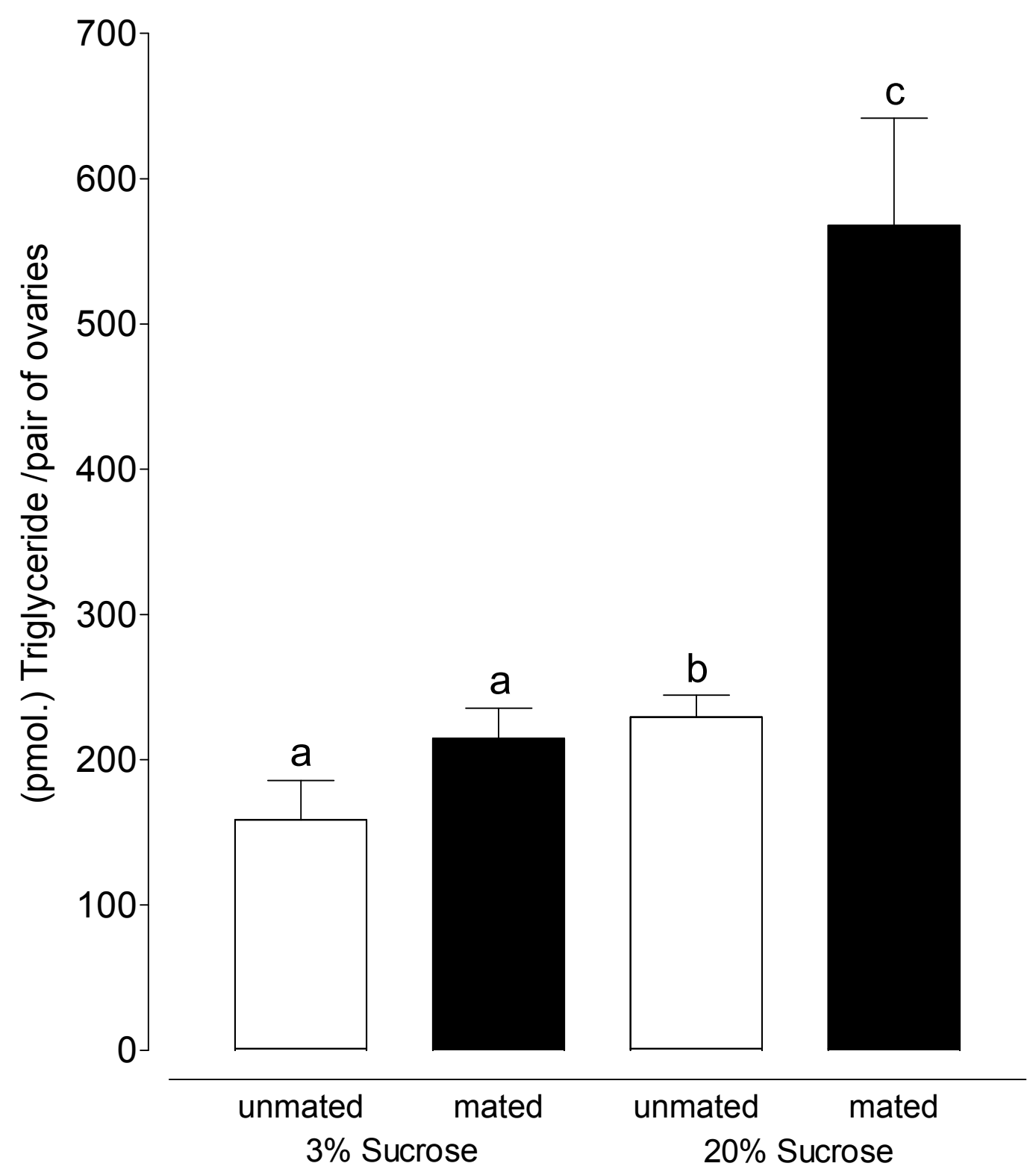

Figure 19: The neutral lipid content of the previtellogenic ovary increases after mating in female mosquitoes maintained on $3 \%$ sucrose. Feeding $20 \%$ sucrose to females and mating causes a 2-fold increase in the neutral lipid content of ovaries. (Mean \pm SEM; Mann-Whitney U-test; Treatments with different letters denote statistical significance at $\mathrm{p} \leq .05)$. 

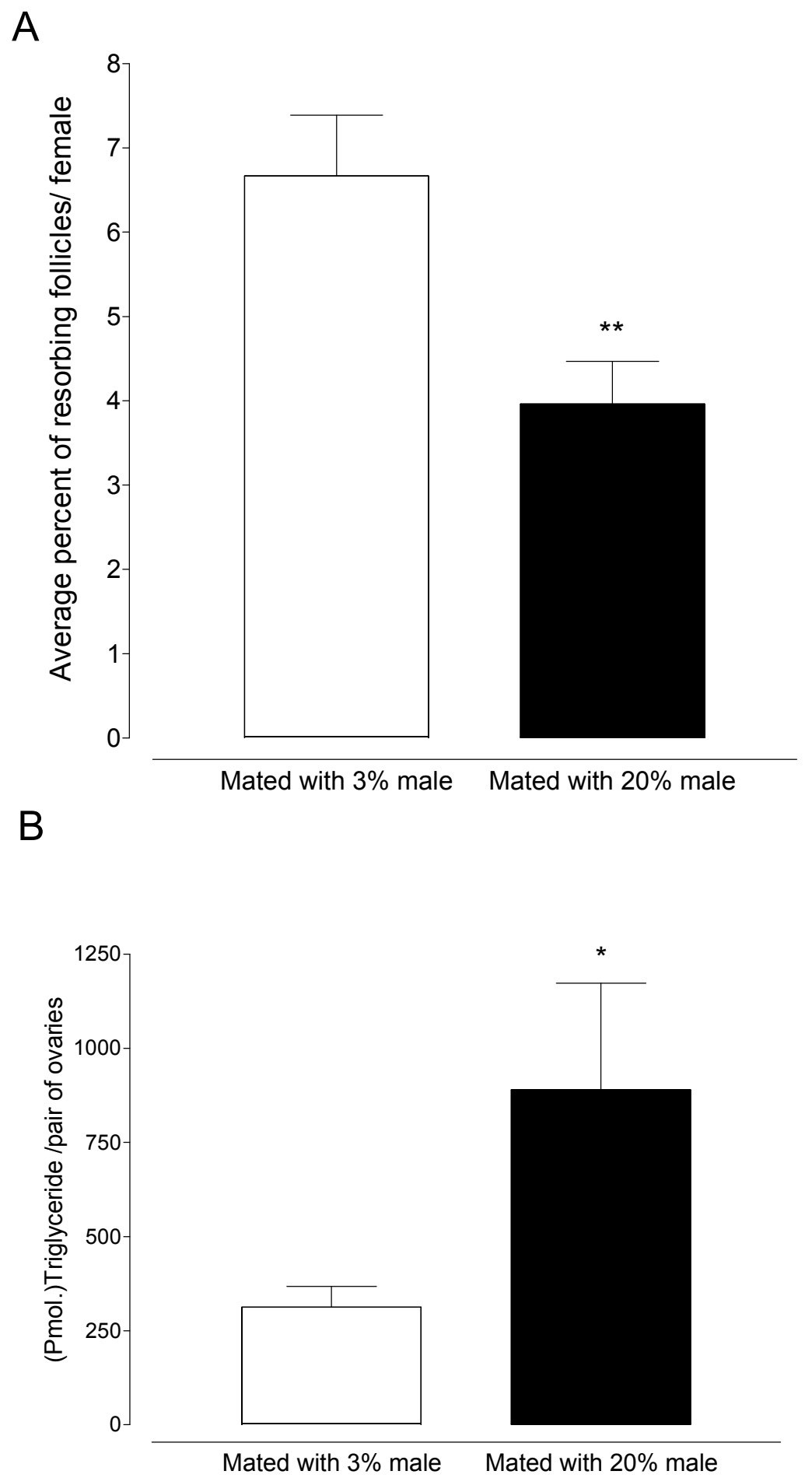

Figure 20: Males maintained on $20 \%$ sucrose exert a larger effect on the rate of follicular resorption and neutral lipid content of ovaries. (A) When females mate with males maintained on $20 \%$ sucrose the rate of resorption decreases by $\sim 40 \%$ (Mean \pm SEM; ${ }^{* *} \mathrm{p} \leq .01$; Unpaired t-test) (B) Mating females with males maintained on $20 \%$ sucrose causes a 3-fold increase in ovarian lipids (Mean \pm SEM; ${ }^{*} p \leq .05$; Mann-Whitney U-test). 


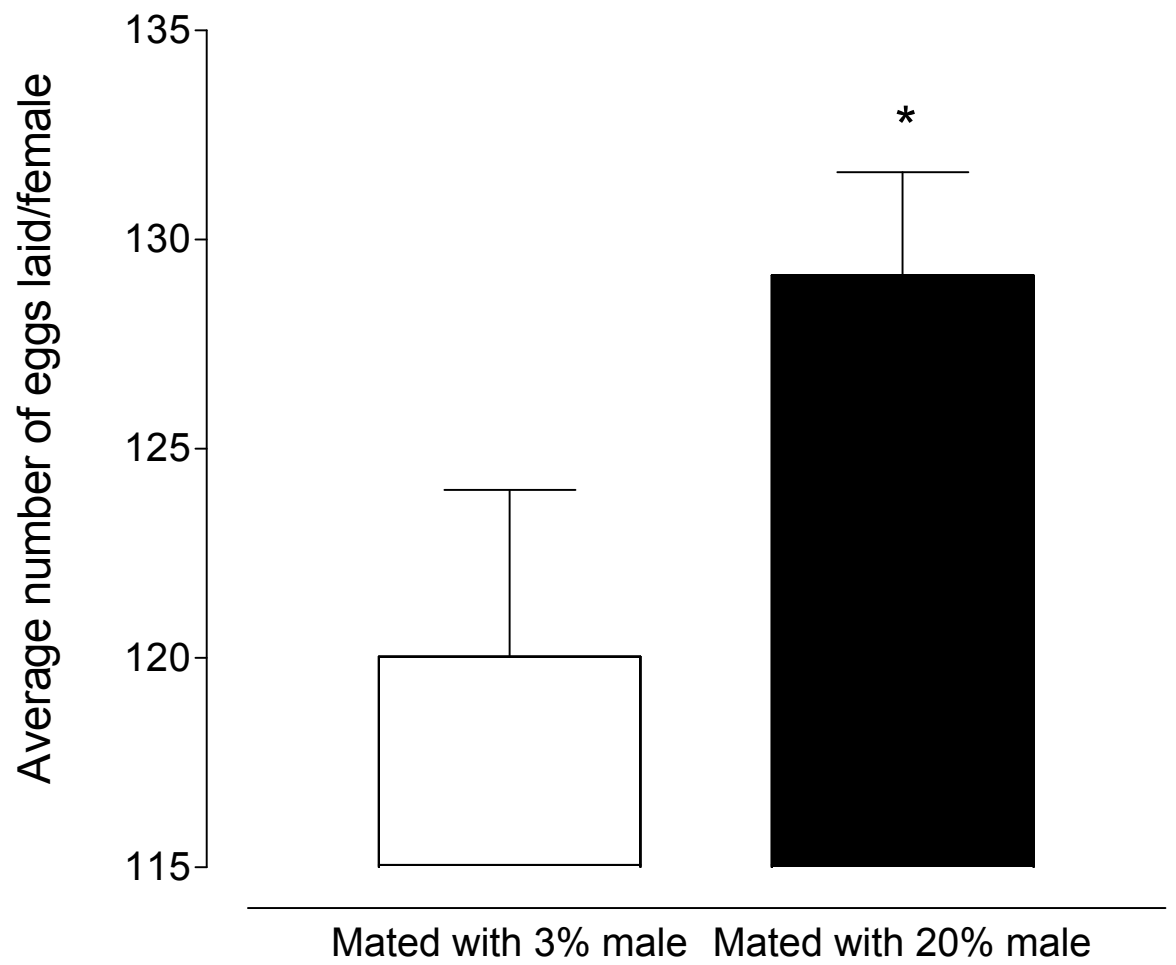

Figure 21: When females are mated to males maintained on $20 \%$ sucrose, reproductive output is increased by $\sim 9$ eggs (Mean \pm SEM ; ${ }^{*} p \leq .05$; Unpaired t-test). 
A

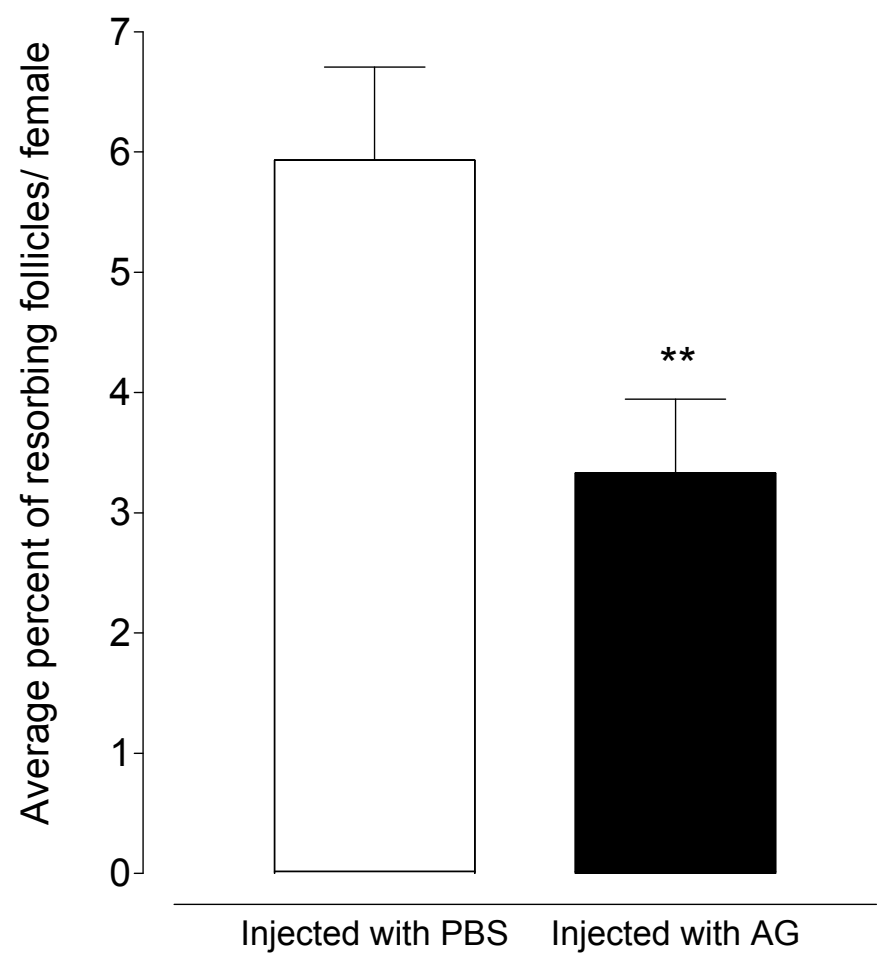

B

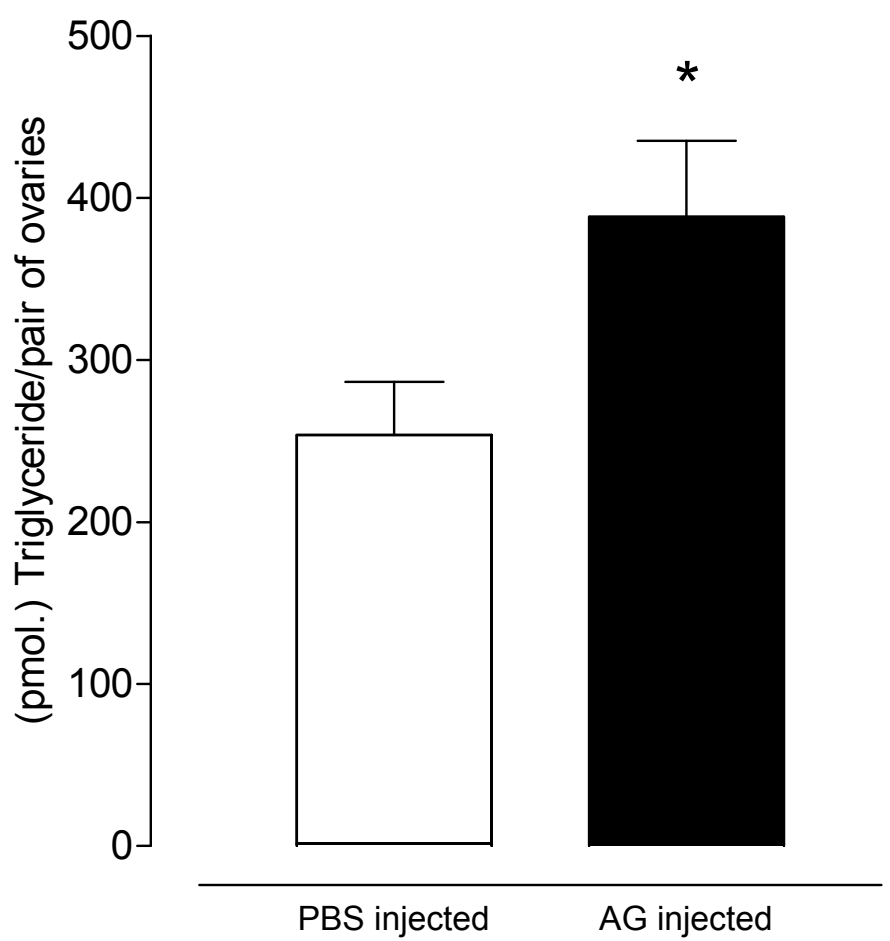

Figure 22: Effect of injected accessory gland homogenates. (A) Accessory gland homogenates reduce resorption (Mean \pm SEM $;{ }^{* *} p \leq .01$; Unpaired t-test) and (B) increase neutral lipid content (Mean \pm SEM ; ${ }^{*} p \leq .05$; Mann-Whitney U-test). 


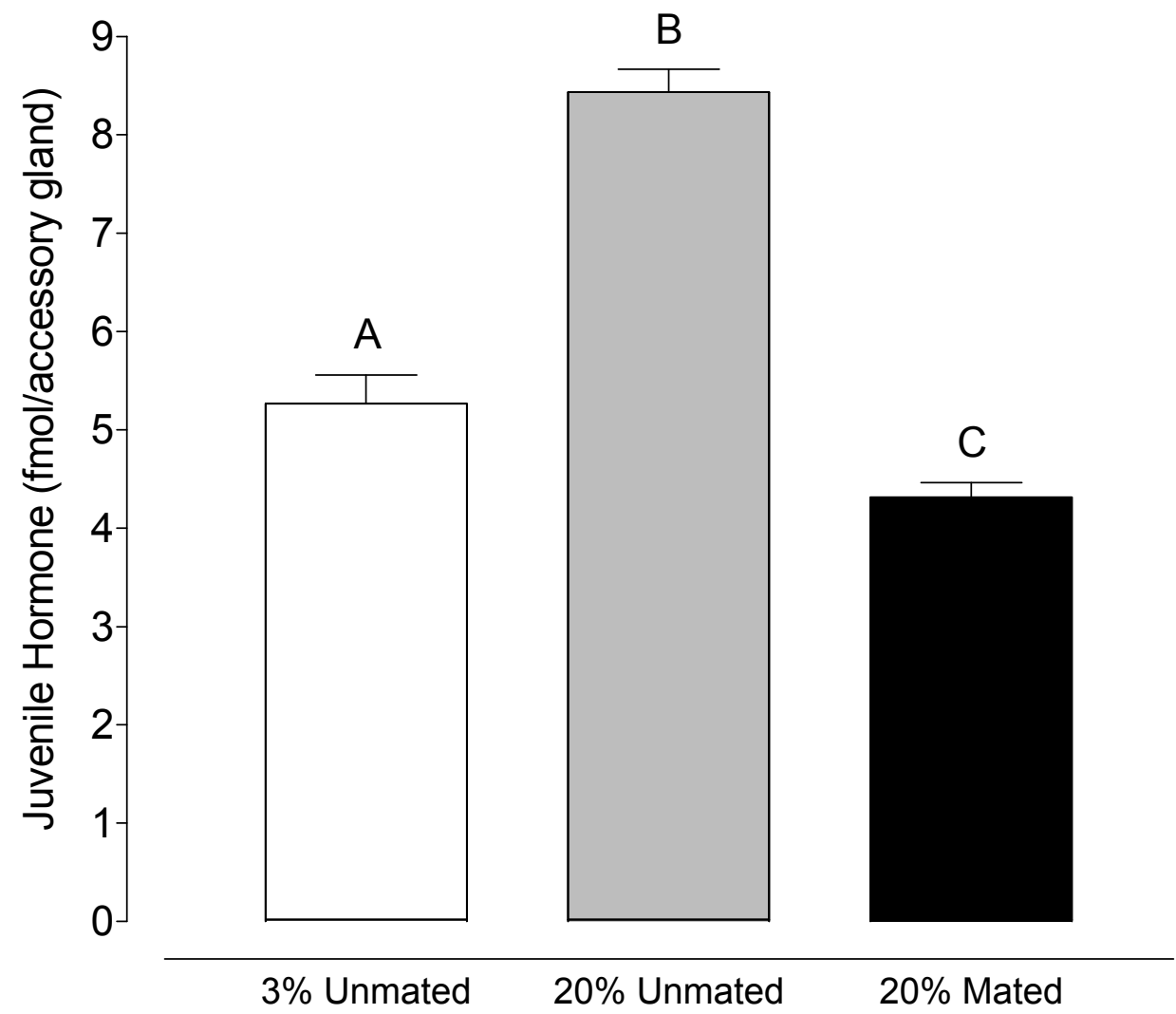

Figure 23: JH III in the accessory gland of virgin males fed $3 \%$ sucrose and $20 \%$ sucrose as well as mated males fed $20 \%$ sucrose. (Mean \pm SEM; Mann-Whitney Utest; Treatments with different letters denote statistical significance at $p \leq .05$ ). 


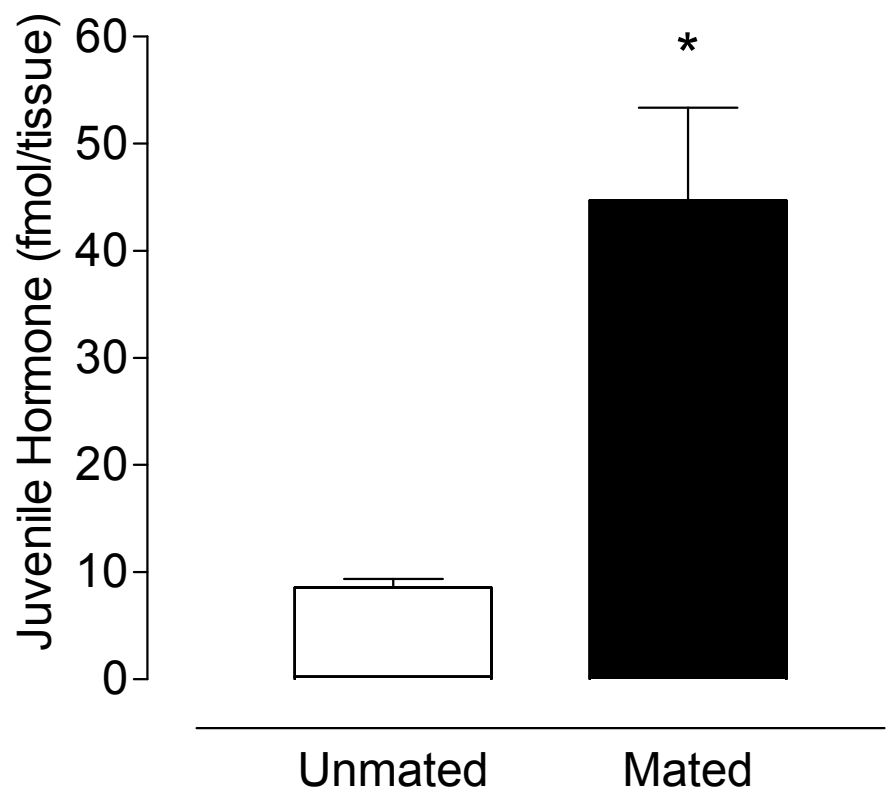

Figure 24: The Bursa copulatrix/Spermathecae complex contains more JH III in mated females than unmated females. (Mean \pm SEM ;* $p \leq .05$; Mann-Whitney U-test). 


\subsection{References}

Boggs, C.L. 1990. A general model of the role of male-donated nutrients in female insects reproduction. American Naturalist. 136, 598-617.

Boggs, C. L. 2009. Understanding insect life histories and senescence through a resource allocation lens. Functional Ecology. 23, 27-37.

Boggs, C.L., Ross, C.L., 1993. The effect of adult food limitation on life-history traits in Speyeria mormonia (Lepidoptera, Nymphalidae). Ecology. 74, 433-441.

Borovsky, D., Carlson, D.A., Hancock, R.G., Rembold, H., van Handel, E. 1994. De novo biosynthesis of juvenile hormone III and I by the accessory glands of the male mosquito. Insect Biochemistry and Molecular Biology. $24,437-444$.

Caroci, A.S., Li, Y., Noriega, F.G. 2004. Reduced juvenile hormone synthesis in mosquitoes with low teneral reserves reduces ovarian previtellogenic development in Aedes aegypti. Journal of Experimental Biology. 207, 2685-2690.

Chen, P.S. 1984. The functional morphology and bio-chemistry of insect male accessory glands and their secretions. Annual Review of Entomology. 29, 233-255.

Clements, A.N., 1992. The Biology of Mosquitoes. Chapman and Hall, London.

Clifton, M.E., Noriega, F.G. 2011.Nutrient limitation results in juvenile hormonemediated resorption of previtellogenic ovarian follicles in mosquitoes. Journal of Insect Physiology. 57, 1274-81.

Clifton, M.E., Noriega, F.G. 2012. The fate of follicles after a blood meal is dependent on previtellogenic nutrition and juvenile hormone in Aedes aegypti. Journal of Insect Physiology. 58, 1007-1019.

Craig, G.B. Jr. 1967. Mosquitoes: female monogamy induced by male accessory gland substance. Science. 156, 1499-501.

de Jong, G., Van Noordwijk, A.J. 1992. Acquisition and allocation of resources genetic (co)variances, selection, and life histories. American Naturalist. 139, 749-770. 
Djawdan, M., Sugiyama, T.T., Schlaeger, L.K., Bradley ,T.J., Rose, M.R. 1996. Metabolic aspects of the trade-off between fecundity and longevity in Drosophila melanogaster. Physiological Zoology. 69,1176-95.

Dottorini, T., Nicolaides, L., Ranson, H., Rogers, D.W., Cristani, A., Catteruccia, F. 2007. A genome-wide analysis in Anopheles gambiae mosquitoes reveals 46 male accessory gland genes, possible modulators off male behavior. Proceedings of the National Academies of Science USA. 104,16215-16220.

Fernandez, N.M., Klowden, M.J. 1995. Male accessory gland substances modify the host- seeking behavior of gravid Aedes aegypti mosquitoes, Journal of Insect Physiology. 41, 965-970.

Flatt, T., Kawecki, T.J. 2007. Juvenile hormone as a regulator of the trade-off between reproduction and life span in Drosophila melanogaster. Evolution. 61, 1980- 1991.

Flatt, T., Tu, M.P., Tatar, M., 2005. Hormonal pleiotropy and the juvenile hormone regulation of Drosophila development and life history. BioEssays. 27, 999-1010.

Foster, W.A., Lea, A.O. 1975. Renewable fecundity of male Aedes aegypti following replenishment of seminal vesicles and accessory glands. Journal of Insect Physiology. 21:1085-1090.

Fox, C.W. 1993. Multiple mating, lifetime fecundity and female mortality of the bruchid beetle, Callosobruchus maculatus (Coleoptera : Bruchidae). Functional Ecology. 7, 203-208.

Gillott, C. 2003. Male accessory gland secretions: Modulators of female reproductive physiology and behavior. Annual Reviews of Entomology. $48,163-184$.

Gwynne, D.T. 1984. Courtship feeding increases female reproductive success in bush crickets. Nature. 307, 361-363.

Gwynne, D.T. 2008. Sexual conflict over nuptial gifts in insects. Annual Reviews of Entomology. 53, 83-101.

Harshman, L.G., Zera, A.J . 2007.The cost of reproduction: the devil in the details. Trends in Ecology and Evolution. 22, 80-86.

Hartfelder, K. 2000. Insect juvenile hormone: from "status quo" to high society. Brazilian Journal of Medical and Biological Research. 33, 157-177. 
Helinski, M.E.H., Harrington L.C. 2011. Male Mating History and Body Size Influence Female Fecundity and Longevity of the Dengue Vector Aedes aegypti Journal of Medical Entomology. 48, 202-211.

Hernandez-Martinez, S., Mayoral, J.G., Li, Y., Noriega FG. 2007. Role of juvenile hormone and allatotropin on nutrient allocation, ovarian development and survivorship in mosquitoes. Journal of Insect Physiology. 53, 230-234

Hiss, E.A., Fuchs, M.S. 1972. The effect of matrone on oviposition in the mosquito Aedes aegypti. Journal of Insect Physiology. 18, 2217-2225.

Judson, C.L. 1967. Feeding and oviposition behavior in the mosquito Aedes aegypti (L.).I. Preliminary studies of physio-logical control mechanisms. Biological Bulletins. 133, 369-377.

Klowden, M.J., Lea, A.O. 1979. Humoral inhibition of host-seeking in Aedes aegypti during oöcyte maturation, Journal of Insect Physiology. 25, 231235.

Klowden, M.J., Russell, R.C. 2004. Mating affects egg maturation in Anopheles gambiae Giles (Diptera : Culicidae). Journal of Vector Ecology. 29, 135139.

Klowden, M.J., Chambers, G.M. 1991. Male accessory gland substances activate egg development in nutritionally stressed Aedes aegypti mosquitoes. Journal of Insect Physiology. 37, 721-726.

Lamb, M.J., 1964. The effect of radiation on the longevity of female Drosophila subobscura. Journal of Insect Physiology. 10, 487-497.

Lavoipierre, M. J., 1958. Biting behaviour of mated and unmated females of an African strain of Aedes aegypti. Nature. 181, 1781-1782.

Li, Y., Hernandez-Martınez, S., Unnithan, G.C., Feyereisen, R., Noriega, F.G., 2003. Activity of the corpora allata of adult female Aedes aegypti: effects of mating and feeding. Insect Biochemistry and Molecular Biology. 33, 1307-1315.

Merle, J. 1968. Ovarian function and sexual receptivity of Drosophila melanogaster after implantation of fragments of the male genital tract. Journal of Insect Physiology. 14, 1159-1168. 
Moshitzky, P., Fleischmann, I., Chaimov, N., Saudan, P., Klauser, S. 1996. Sex peptide activates juvenile hormone biosynthesis in the Drosophila melanogaster corpus allatum. Archives of Insect Physiology and Biochemistry. 32, 363-74.

Moczek, A.P., 1998. Horn polyphenism in the beetle Onthophagus taurus: larval diet quality and plasticity in parental investment determine adult body size and male horn morphology. Behavioral Ecology. 9, 636-641.

Moczek, A.P., Nijhout, H.F., 2004. Trade-offs during the development of primary and secondary sexual traits in a horned beetle. The American Naturalist. $163,184-191$.

O'Meara, G. F., Evans, D. G. 1976. The influence of mating on autogenous egg development in the mosquito, Aedes taeniorhynchus. Journal of Insect Physiology. 22, 613-617.

O'Meara, G. F., Evans, D. G. 1977. Autogeny in salt marsh mosquitoes induced by a substance from the male accessory gland. Nature. 267, 342-344.

Ohgushi, T., 1996. A reproductive tradeoff in an herbivorous lady beetle: egg resorption and female survival. Oecologia. 106, 345-351.

Oliveira, G.A., Baptista, D.L., Guimaraes-Motta, H., Almeida, I.C., Masuda, H., Atella, G.C., 2006. Flight-oogenesis syndrome in a blood sucking bug: biochemical aspects of lipid metabolism. Archives of Insect Biochemistry and Physiology. 62, 164-175.

Osawa, N., 2005. The effect of prey availability on ovarian development and oosorption in the ladybird beetle Harmonia axyridis (Coleoptera: Coccinellidae). European Journal of Entomology, 102, 503-511.

Park, Y.I., Shu, S., Ramaswamy, S.B., Srinivasan, A. 1998. Mating in Heliothis virescens: transfer of juvenile hormone during copulation by male to female and stimulation of biosynthesis of endogenous juvenile hormone. Archives of Insect Physiology and Biochemistry. 38,100-107.

Partridge, L., Green, A., Fowler, K. 1987. Effects of egg-production and of exposure to males on female survival in Drosophila melanogaster. Journal of Insect Physiology. 33, 745-749. 
Pondeville, E., Maria, A., Jacques, J.C, Bourgouin, C., Dauphin-Villemant, C. 2008. Anopheles gambiae males produce and transfer the vitellogenic steroid hormone 20-hydroxyecdysone to females during mating. Proceedings of the National Academies of Science USA. 105, 1963119636.

Sirot, L.K., Poulson, R.L., CaitlinMcKenna, M., Girnary, H., Wolfner, M.F., Harring ton, L.C. 2008. Identity and transfer of male reproductive gland proteins of the dengue vector mosquito, Aedes aegypti: potential tools for control of female feeding and reproduction. Insect Biochemistry and Molecular Biology. 38,176-189.

Sirot, L.K., Hardstone, M.C., Helinski, M.E., Ribeiro, J.M., Kimura M., Deewatthanawong, P., et al. 2011. Towards a semen proteome of the dengue vector mosquito: protein identification and potential functions. PLoS Neglected Tropical Diseases. 5:e989; PMID:21423647.

Socha, R., Sula, J. 2008. Differential allocation of protein resources to flight muscles and reproductive organs in the flightless wing-polymorphic bug, Pyrrhocoris apterus (L.) (Heteroptera). Journal of Comparative Physiology. B, Biochemical Systemic and Environmental Physiology. 178: 179-188.

Spielman, A., Leahy, M.G., Skaj, V. 1967. Seminal loss in repeatedly mated female Aedes aegypti. Biological Bulletins. 132, 404-412.

Tatar, M., Carey, J.R. 1995. Nutrition mediates reproductive trade-offs with age specific mortality in the beetle Callosobruchus maculatus. Ecology. 76, 2066-73.

Thornhill, R. 1976. Reproductive behaviour of the love bug, Plecia nearctica (Diptera: Bibionidae). Annals of the Entomological Society of America. 69, 843-847.

Uchida, K., Moribayashi, A., Matsuoka, H., Oda, T., 2003. Effects of mating on oogenesis induced by amino acid infusion, amino acid feeding, or blood feeding in the mosquito Anopheles stephensi (Diptera: Culicidae). Journal of Medical Entomology. 40, 441-446.

Vahed, K. 1998. The function of nuptial feeding in insects: a review of empirical studies. Biological Reviews. 73,43-78.

Wolfner, M. F. 1997. Tokens of love: functions and regulation of Drosophila male accessory gland products. Insect Biochemistry and Molecular Biology. 27, 179-192. 
Wolfner, M.F. 2002. The gifts that keep on giving: physiological functions and evolutionary dynamics of male seminal proteins in Drosophila. Heredity. $88,85-93$.

Wolfner M.F. 2009. Battle and ballet: molecular interactions between the sexes in Drosophila. Journal of Heredity. 100: 399-410.

Yeh, C.C., Klowden, M.J. 1990. Ejects of male accessory gland substances on the pre-oviposition behaviour of Aedes aegypti mosquitoes. Journal of Insect Physiology. 36, 799-803.

Zera, A.J., Brink, T. 2000. Nutrient absorption and utilization by wing and flight muscle morphs of the cricket Gryllus firmus: implications for the trade-off between flight capability and early reproduction. Journal of Insect Physiology. 46, 1207-18. 


\section{CHAPTER 6: Conclusions and Future directions}

\subsection{Conclusions}

As described in chapter 1 the primary aims of this dissertation included determining the relationships between juvenile hormone, nutritional status and the effect of mating and male contributions on follicular resorption and reproductive output. The accomplish this goal the research presented here: 1) determined the relationship between juvenile hormone, nutritional status and fitness trade-offs during the previtellogenic stage within the context of life-history theory, 2) described a model that integrates the endocrinology of reproductive physiology before a blood meal with fitness trade-offs after a blood meal and 3) used this model to explain the male contribution to reproduction and fecundity.

By experimental extending the previtellogenic resting stage and offering mosquitoes various concentrations of sucrose, it became clear that extensive fitness trade-offs were occurring through the process of follicular resorption. Some other observations from chapter 3 include:

1) The rate of follicular resorption in resting stage mosquitoes is dependent on nutritional quality.

2) Follicular resorption is related to $\mathrm{JH}$ levels. Decrease in the nutritional status causes decrease in $\mathrm{JH}$ levels and increase in follicular resorption.

3) An apoptotic cell-death mechanism is responsible for follicular resorption during the previtellogenic resting stage.

4) $\mathrm{JH}$ mediates reproductive trade-offs in resting stage mosquitoes in response to nutrition. 
Nutritional and hormonal manipulations during the resting stage also produced ovarian phenotypes beyond only resorption. By characterizing aspects of ovarian phenotypes which differ in response to sugar feeding and hormonal treatments, it was determined that $\mathrm{JH}$ not only mediates resorption but also mediates the intrinsic quality of follicles. With these observations a model was constructed (Figure 16) that explained how $\mathrm{JH}$ can mediate reproductive tradeoffs both before and after a blood meal. Chapter 4 also determined that:

1) The lipid content of the previtellogenic ovary reflects overall nutritional reserves.

2) Accumulation of ovarian mRNA's integral to oogenesis depend on nutrition and hormones.

3) $\mathrm{JH}$ III treatment during the previtellogenic stage affects post blood meal resorption.

4) Nutrition during the previtellogenic stage also affects post blood meal resorption.

5) $\mathrm{JH}$ mediates reproductive output by affecting a follicles development potential.

Much previous literature has described the effect of mating as "enhancing" or "activating" reproduction. By investigating the "enhancing" effect of the male contribution using the physiological markers of ovarian quality developed in chapter 4 , it was determined that male mosquitoes can contribute to fecundity likely by transferring $\mathrm{JH}$ III during copulation and altering the endogenous resource allocation balance with the female. Chapter 5 also determined that: 
1) Mating alters markers of ovarian quality that are correlated with reproductive output such as the rate or resorption and neutral lipid content.

2) The effect of mating on ovarian quality is caused by substances within the male accessory gland.

3) Offering higher sucrose concentrations to males increased the magnitude of the effect on female ovarian physiology which leads to an increase in the reproductive output of females.

4) The male accessory gland contains JH III.

5) The bursa copulatrix/spermathecae complex of a mated female contains more $\mathrm{JH}$ III than that of an unmated female indicating a likely transfer of JH III.

Together, the observations presented here demonstrate that reproductive output in the female mosquito is a function of previtellogenic sugar feeding, blood meal derived nutrition and is also dependent on the male contribution during copulation. It is also clear from these results that $\mathrm{JH}$ III rests at the center of the mechanism that determines reproductive output during the previtellogenic resting stage.

\subsection{Future Directions}

Future research on this topic will be focused around two important aims: 1) Understanding how resources recovered from follicular resorption are re-used in insects and also 2) describing the molecular and biochemical mechanisms behind follicular resorption. Follicular resorption represents a mechanism in which the flow of resources allocated towards reproduction seems to be stopped (or even reversed towards somatic processes as some evidence has suggested) and represents a unique and surprisingly unstudied exception to traditional 
models of resource allocation and the germ/soma relationship. No model of resource allocation exists that incorporates the consequences of follicular resorption nor has the molecular basis for resorption been characterized despite the potential benefits of such work for insect control.

While it has been assumed that resorption is a mechanism that can extend survival and can act as a hedge against stochasticity in the environment, resorption still occurs in some insects when conditions are suitable. This suggests that the reasons for resorption are counterintuitive and more complicated that previously supposed. To understand how follicular resorption fits into existing theories of resource allocation, there are three hypotheses that can explain the fate of resources recovered during resorption:

1) To support immediate physiological needs (survival, soma, maintenance).

2) To enhance the quality of other follicles in a quality vs. quantity tradeoff.

3) To reduce expenditures on existing reproductive tissues.

Each of these hypotheses have vastly different implications for our understanding of resource allocation, the germ/soma relationship as well as the reasons for resorption. Although some evidence exists to support each of these possibilities without excluding the others, the hypotheses suggested here have never been explicitly tested.

Future research directions also include understanding the molecular mechanisms that govern resorption. To support this future research the transcriptome of the mosquito ovary has been sequenced under various 
experimental conditions including mated and unmated mosquitoes as well as mosquitoes maintained under different nutritional regimes. From this experimental design, the nutritional and hormonal factors that determine reproductive output should be able to be separated. Also, the ovary transcriptome may yield some clues about how resources recovered from resorption are being reused. However, the analysis of the results from this experiment is only just beginning. Ultimately, the transcriptome library in conjunction with the experimental system of resorption developed in this dissertation will be used to examine the molecular mechanisms behind follicular resorption and continue this line of research . 


\section{MARK ELLIS CLIFTON}

September 15th, 1979 2007

2008-2012
Born, New London, Connecticut

B.S. Biology Eastern Connecticut State University Willimantic, Connecticut Doctoral Candidate Florida International University Miami, Florida

Teaching Assistant Florida International University Miami, Florida

\section{Papers and Presentations}

Clifton, M.E. and F.G. Noriega. 2012. The fate of follicles after a blood meal is dependent on previtellogenic nutrition and juvenile hormone in Aedes aegypti. Journal of Insect Physiology, 58 (7): 889-1036.

Clifton, M. E. and F.G. Noriega. Nutrition and juvenile hormone during the resting stage alters follicles quality and vitellogenic development. Sixth International Symposium on Molecular Insect Science, 2nd-5th October 2011, Amsterdam, The Netherlands.

Clifton, M.E. and F.G. Noriega. 2011. Nutrient limitation results in juvenile hormone-mediated resorption of previtellogenic ovarian follicles in mosquitoes. Journal of Insect Physiology, 57(9): 1274-1281.

Clifton, M.E. and F.G. Noriega. Juvenile hormone, a nutrient sensor that controls reproduction in the yellow fever mosquito (Aedes aegypti). $13^{\text {th }}$ annual FIU Biomedical and comparative Immunology Symposium. March $3^{\text {rd }}-4^{\text {th }} 2011$ Florida International University, Miami, Florida.

Clifton, M.E. and F.G. Noriega. Juvenile hormone, a nutrient sensor that controls 
reproduction in the yellow fever mosquito (Aedes aegypti). $13^{\text {th }}$ annual FIU Biological Research Symposium. January 29 ${ }^{\text {th }}, 2011$ Florida International University, Miami Florida.

Edwards, M., Nouzova, M., Clifton M.E. , and F.G. Noriega. Does ecdysone play a role in the regulation of $\mathrm{JH}$ synthesis in mosquitoes? The $18^{\text {th }}$ International Ecdysone Workshop. July $19^{\text {th }}-23^{\text {rd }} \quad 2010$. Ceske Budejovice, The Czech Republic. 